\title{
Proceedings of the First Neurological Disorders Summit (NDS-2015)
}

\section{$\underline{\text { Keynote Abstracts }}$}

\section{Biologically Based Genomic Therapy for Brain Reward Deficiency Syndrome: Are We Going to the Promised Land}

\author{
Kenneth Blum ${ }^{\square}$ \\ University of Florida, USA
}

\begin{abstract}
Introduction: Since the time of Jellinek and his "disease concept of alcoholism" the addiction scientific literature has exploded and we are beginning to not only understand the neurobiological and genetic basis of all addictive behaviors but plausible treatments. Through the advent of neuroimaging techniques we are getting closer to linking neurochemical and neurogenetic mechanisms to reward circuitry brain function. Through candidate and GWAS approaches many gene polymorphisms and clusters have been implicated in drug, food and behavioral dependence linked to a common rubric termed Reward Deficiency Syndrome (RDS). Recognizing the clinical benefit of early genetic testing for determination of addiction risk stratification and coupling dopaminergic agonistic rather than antagonistic therapies potentially genomic based is the wave of the future. The results of studies including gene therapy in animal models, epigenetics and even the role of non-coding micro RNAs reviewed in this presentation provides a frame work for potential genomic based therapies, but none to date has given rise to human trials. Certainly further development especially in gene transfer work and viral vector identification could make gene therapy for RDS a possibility in the future.
\end{abstract}

Areas Covered: This presentation reviews the previously published basic research and clinical trials involving both the neurogenetics and gene transfer work related to many addictions including alcohol, cocaine, opioids, nicotine, glucose, feeding, gambling, internet gaming subsets of RDS. We briefly review the findings related to animal models that incorporate gene therapy providing a discussion of positive and negative results. We also briefly list a number of other treatment alternatives as well such as deep brain stimulation, acupuncture, pharmacogenomics and nutrigenomics alternative methods to manipulate transcription and gene expression thereof.

Expert Opinion: Our basic tenant is that since addiction albeit its high heredity component, is based on reward deficiency and a hypodopaminergic trait, does not follow Mendelian inheritance (sui generis) and as such it is unrealistic to expect an addiction gene therapy to emerge soon. The major problem is that unlike peripheral disorders, that are monogenetic-one gene one disease, RDS and all subtypes are polygenic in nature. Thus it does not allow clinicians to simply swap a "disease gene" for a so called "healthy one" ultimately leading a "cure". Furthermore, it is the current nemesis of addiction medicine specialists not to be able to establish appropriate "nosological entities" to help identify risk in our youth. While the future seems bright with advent of genetic testing, before gene therapy in addiction can be considered in humans many neurobiological and technical problems must be overcome, as well as ethical questions such as permanently changing ones basic innate characteristics that makes us diverse and human.

\section{Recent Progresses on Neuroimaging with Ultra-high Field MR}

\author{
Zang-Hee Cho ${ }^{*}$ \\ Seoul National University, Korea
}




\begin{abstract}
New imaging system, the Brain dedicated PET-MRI, using High resolution PET and Ultra High Field 7.0T Magnetic Resonance Imaging (MRI) and their applications to basic and clinical neuroscience, especially to the areas of neuropsychiatry, and neurological disorders will be discussed. Among the interesting topical areas, applications of the high resolution brain PET(HRRT) and the ultra-high field MRI(7.0T) will be highlighted, especially for the in vivo, human brain imaging. With high field MRI, such as the 7.0T MRI, one can new visualize the subfields of the hippocampus and brainstem in-vivo as well as tractography hitherto unable to do with existing MRI systems. Together with molecular imaging using Positron Emission Tomography (PET), that is the brain dedicated PET-MRI fusion system developed recently, now, it is possible to visualize molecular mechanisms quantitatively in our human brain in-vivo as well as connectivity through the tractography. Especially, Ultra-high field MRI also began to provide excellent tractographic images delinealing fine fibers such as medial forebrain bundles and internal medullary laminars in the thalamo-limbic areas suggesting future potential applications of these fibers to, among others, for example in the DBS. Some recent results of brain PET-MRI fusion system as well as the new tractographic image obtained with 7.0T will be discussed and highlighted.
\end{abstract}

\title{
Intranasal Small Molecules, Proteins, Oligonucleotides and Stem Cells Bypass the Blood-brain Barrier and Target the Brain to Treat Alzheimer's, Parkinson's, Stroke, Brain Tumors, MS and Other Brain Disorders
}

\author{
William H. Frey II" \\ University of Minnesota, USA
}

\begin{abstract}
This presentation will focus on the non-invasive intranasal method of bypassing the blood-brain barrier (BBB) to deliver and target therapeutics to the CNS to treat a variety of disorders. Therapeutics include small molecules, peptides, proteins, oligonucleotides, stem cells and other therapeutic cells. This delivery, targeting and treatment method relies on rapid extracellular delivery along the olfactory and trigeminal neural pathways from the nasal mucosa to the brain and, once inside the brain, on the perivascular pump mechanism of the cerebral vasculature that can rapidly move substances throughout the CNS. Intranasal delivery not only delivers a variety of therapeutics to the CNS but also reduces systemic exposure and unwanted side effects. Different formulations have also be used to facilitate the delivery of poorly water soluble therapeutics, to further reduce systemic exposure to therapeutics and to enhance delivery of therapeutics to the CNS. Nasal drug delivery devices have been developed to facilitate delivery to the upper third of the nasal cavity to enhance delivery of therapeutics to the CNS. Using this method, intranasal therapeutics including adult stem cells have been shown to treat stroke, Parkinson's, Alzheimer's, brain tumors, MS and other disorders in animal models. Intranasal insulin has been shown in multiple phase 2 clinical trials to improve memory in both normal adults and in patients with mild cognitive impairment or Alzheimer's disease with no change in the blood levels of insulin or glucose. Intranasal delivery is changing the way we treat CNS disorders.
\end{abstract}

\section{Genomics View of Neurodegenerative Diseases}

\author{
Shoji Tsuji" \\ The University of Tokyo Hospital, Japan
}

\begin{abstract}
The majority of neurodegenerative diseases are observed as "sporadic" diseases. Despite remarkable progresses in molecular genetics of neurodegenerative diseases with Mendelian trait, molecular bases of sporadic neurodegenerative diseases remain largely unknown. Previous epidemiological studies, however, strongly support genetic bases for these diseases. As potential molecular bases, various mechanisms can be considered, which include those underlying apparently sporadic diseases with low penetrant mutations, those with de novo mutations, and those associated with variants in disease-susceptible genes. The availability of high-throughput genome sequencing employing next generation sequencers is expected to revolutionize our understanding of not only hereditary but also sporadic neurodegenerative diseases. High-throughput genome sequencing technologies will enable us to explore all of these possibilities with an unprecedentedly robust power. Various research paradigms can be applied to identify disease-susceptibility genes, which include 1. Data-driven approach (e.g. whole-exome association studies), 2. Genetics-based approach focusing on multiplex families and 3. Hypothesis-driven approach based on biological knowledge. Employing the genetics-based approach, we have recently been able to identify a gene associated with familial as
\end{abstract}


well as sporadic multiple system atrophy (MSA). Future perspective toward better understanding molecular bases of sporadic diseases will be discussed.

\title{
Beta Adrenergic Signaling in Down Syndrome and Alzheimer's Disease
}

\author{
Ahmad Salehi \\ Stanford Medical School, USA
}

\begin{abstract}
Down syndrome (DS) is the primary genetic cause of learning disabilities in humans. Furthermore, almost all adults with DS will develop Alzheimer's disease pathology. The hippocampal region undergoes significant degeneration and dysfunction in DS. For this reason, we have focused on this region and brain areas that extensively innervate the hippocampus. Our studies have shown that the hippocampal region with significant role in cognitive function undergoes atrophy and dysfunction in mouse models of DS. To identify the neurobiological basis of hippocampal dysfunction in DS, we have focused on the locus coeruleus. We found that norepinephrine-ergic (NE) neurons in the locus coeruleus, undergo significant degeneration in the Ts65Dn mouse model of DS. Accordingly, increasing brain NE levels can restore cognitive function in the Ts65Dn mouse model of DS. To expedite the process of drug development for cognitive dysfunction in DS, we have focused on drugs already in the market. Our investigations have indicated that two already approved drugs, i.e. L-DOPS (an NE pro-drug) and formoterol (a long-acting beta2 adrenergic receptor agonist), can significantly improve contextual learning in the Ts65Dn mouse model of DS. Currently, we are studying the effects of formoterol and L-DOPS on cognitive dysfunction and testing ways to increase their safety and efficacy
\end{abstract}

\section{Multiscale Genomic Approach and Big Data Analysis Support a Shared Etiology between Type 2 Diabetes and Alzheimer's Disease}

\author{
Giulio Maria Pasinetti ${ }^{1,2}$ \\ ${ }^{1}$ Icahn School of Medicine at Mount Sinai, USA \\ ${ }^{2}$ James J. Peters Veterans Affairs Medical Center, USA
}

\begin{abstract}
Many epidemiological studies indicate that people with diabetes are at higher risk of developing Alzheimer's disease (AD) or other dementias. A massive longitudinal database involving at-risk populations is an essential infrastructure needed to address the future needs of a major prevention initiative. Along with 'Big-Data', the field of therapy development will require novel computational capabilities to not only sort out the complex interactions between type 2 diabetes (T2D) and cognitive deterioration in $\mathrm{AD}$, but also to discover-validate technologies for the early and accurate detection of the disease. We used data from public genome-wide association studies (GWAS) to explore the association single-nucleotide polymorphisms (SNPs) between T2D and AD. Next, we explored the function of the T2D-AD shared GWAS SNPs by integrating pathway data with gene ontology data, expressional quantitative trait loci (eQTL), and co-expression networks. We found a significant overlap $(\mathrm{p}=4.9 \mathrm{E}-19)$ between association SNPs from large-scale GWAS of T2D and AD. 927 SNPs were associated with both T2D and T2D with $\mathrm{p} \leq 0.01$, and we found that these SNPs influence 190 genes in brain tissue and 416 genes in T2D-relevant peripheral tissues (liver and adipose). Interestingly, we found that mitochondrial functions and innate immune response pathway genes were significantly enriched. In conclusion, we found that T2D and AD share common genetic risk factors, which may partially explain the epidemiological observation of disease incidence correlation.
\end{abstract}

\section{Imaging Neurotransmission in the Live Human Brain}

\author{
Rajendra D. Badgaiyan $\square$ \\ University of Minnesota, USA
}

\begin{abstract}
Neuroimaging techniques (fMRI and PET) have made significant contribution to our understanding of the brain mechanisms that control human cognition and behavior. These techniques provide precise spatial and temporal information
\end{abstract}


about task induced brain activations but cannot reliably detect neurochemical changes associated with the brain processing. Lack of this information has significantly limited our understanding of human cognitive control. It has also restricted our knowledge of the pathophysiology of psychiatric and neurological conditions. We have recently developed a dynamic molecular imaging technique to detect, map and measure task-induced changes in dopamine neurotransmission in the live human brain. In these experiments we exploit the competition between endogenously released neurotransmitter and specific receptor ligand for receptor occupancy. The technique expands the scope of neuroimaging research and allows investigation of an unexplored aspect of human cognition and behavior.

\title{
Biomarkers-directed Strategies to Prevent, Early Diagnose and Treat Autism
}

\author{
Afaf El-Ansary ${ }^{1,2}$ \\ ${ }^{1}$ King Saud University, Saudi Arabia \\ ${ }^{2}$ Autism Research and Treatment Center, Saudi Arabia
}

\begin{abstract}
Autism is associated with deficits in social interaction and communication, restricted interests and repetitive behaviors. Signs of autism are not evident in the first six months of life, but emerge in the second half of the first year. Literature suggests that the prevalence of ASD has increased 20 times, from a rate around 1:2500 in the mid 1980s to a rate of 1:50 at 2014. Currently, many environmental factors, both pre- and postnatal, are found to be associated with ASD. Many autistic children have multiple medical problems including, oxidative stress and glutathione depletion, decreased methylation; mitochondrial dysfunction; intestinal dysbiosis showing increased shifted toward an overgrowth of Clostridia species, toxic metal burden; immune dysregulation, characterized by elevation of proinflammatory cytokines and a unique inflammatory bowel disease and immune activation of neuroglial cells and glutamate excitotoxicity. Biomarkers should ideally be non-invasive quantitative biological or biochemical measures with an accurate indication of a specific disease mechanism. Identifying biomarkers will almost certainly leading to a better understanding of the pathogenesis required to design the most effective preventive, diagnostic and treatment strategies for autism. Based on our understanding of different mechanisms involved in the etiology of autism, pregnant mothers and balanced at-birth gut microbiota will be discussed as target for preventive strategies. Oxidative stress, neuroinflammation, neurochemical and excitotoxicity related markers will be traced as biomarkers-guided early diagnosis strategy. Moreover, success of the use of hyperbaric oxygen treatment (HBOT), memantine, oxytocin, nutritional strategies (e.g. probiotic antioxidants, $\mathrm{N}$-acetyl cysteine, melatonin and supplemental glutamine as a treatment strategy to treat autism will be reviewed.
\end{abstract}




\title{
Speaker Abstracts
}

\section{Gene-environment Interactions, Experience-Dependent Plasticity and Therapeutic Targets for Neurological Disease}

\author{
Anthony J. Hannan ${ }^{1,2, \varpi, \AA}$, Thibault Renoir ${ }^{1}$, Christina Mo ${ }^{1,3}$, Dean Wright ${ }^{1}$, Xin Du ${ }^{1}$ and Terence Y. Pang ${ }^{1}$ \\ ${ }^{1}$ Florey Institute of Neuroscience and Mental Health, University of Melbourne, Australia \\ ${ }^{2}$ Department of Anatomy and Neuroscience, University of Melbourne, Australia \\ ${ }^{3}$ Department of Neurobiology, University of Chicago, USA
}

\begin{abstract}
Huntington's disease (HD) is a tandem repeat (CAG) expansion disorder involving psychiatric, cognitive and motor symptoms. In a transgenic mouse model of HD, we have shown that expansion of the polyglutamine tract of the mutant huntingtin protein leads to a spatiotemporally specific cascade of molecular, cellular and behavioural abnormalities. We have also demonstrated that environmental enrichment (which enhances sensory stimulation, cognitive activity and physical exercise) can delay onset of the affective, cognitive and motor endophenotypes. Environmental enrichment and physical exercise induce changes in gene expression, which exhibit temporal specificity and regional selectivity. Our results suggest that the timing and duration of these environmental manipulations are critical in terms of their ability to modify gene expression. Detailed investigations of these HD mice have revealed depression-like behaviours and cognitive deficits (modelling dementia), which precede motor deficits. The female HD mice demonstrate early affective abnormalities which can be rescued by administration of clinically effective antidepressant drugs as well as increased physical exercise. We have thus been able to investigate these mice as a model of depression and HD, and have discovered various molecular abnormalities. These findings have been extended to additional environmental factors (including the negative effects of stress), neuroendocrine modulators and animal models of other brain disorders. Together with epidemiological studies and clinical trials, this research is informing the design of ongoing intervention studies for HD. These approaches may also facilitate the development of 'enviromimetics' for a variety of brain disorders known to be modulated by enhanced cognitive activity and physical exercise.
\end{abstract}

\section{Transthyretin and the Nervous System: Friend or Foe?}

\author{
Joel N. Buxbaum \\ The Scripps Research Institute, USA
}

\begin{abstract}
It has been known for more than 30 years that amino acid substitutions in the serum and CSF protein transthyretin (TTR) have been associated with an autosomal dominant sensorimotor and autonomic neuropathy initial called Familial Amyloidotic Polyneuropathy type 1 or Portuguese type. Since the initial description 113 amino acid substitutions in the 127 amino acid protein have been reported to be associated with this syndrome or Familial Amyloidotic Cardiomyopathy, a related disorder in which the major target organ is the heart. These are gain of toxic function rather than loss of function disorders. The normal function of the protein was thought to be that of a carrier of vitamin A systemically and thyroxine (T4) to the brain during development. However, silencing of both copies of the endogenous murine gene resulted in a mildly abnormal behavioral phenotype with considerable disruption of the hippocampal CA3 region and a marked diminution in neuroblast numbers in the supraventricular zone. It is not clear how much of this is developmental and how much is somatically based. In addition it has been noted that peripheral nerve regeneration after injury is dependent on the presence of TTR which appears to be synthesized primarily by Schwann cells. Stimulated by early studies showing that TTR was one of the three A $\beta$-binding proteins in the CSF we carried out experiments designed to explore the relationship between TTR and neurodegenerative disease. We have shown that over-expression of wild type human TTR suppresses the behavioral and neuropathologic AD-like features in the APP23 model mouse with TTR-A $\beta$ complexes present in the brains of those mice as well as some human AD brains and that TTR is over-expressed in the neurons of APP23 mice. We have also found that $70 \%$ of cortical and hippocampal neurons in human AD (compared with 10\% in age and gender matched) brains stain with an antibody to TTR as do all the neurons in the APP23 mouse brains and under these circumstances neuronal TTR expression appears to be regulated by the major stress response element HSF1. In examining other human and murine models of neurodegenerative diseases including murine prion disease, Lewy Body disease, mice bearing mutated forms of human $\alpha$-synuclein and rats transgenic for the HIV gp120 protein we have also found markedly increased neuronal staining for TTR. It is our hypothesis that neuronal synthesis of TTR represents an element of defense against a variety of neurological insults. TTR appears to be a multifunctional protein, based on
\end{abstract}


its capacity to bind a variety of chemical structures, rendering them non-toxic. In humans it is a genetically relatively conserved protein implying that disruption of its structure is not well tolerated. It is possible that TTR is one member of a family of multifunctional, non-ATP requiring proteins utilized by the nervous system to defend itself at little energy cost that might be broadly exploitable therapeutically or in prophylaxis.

\title{
Brain-derived Neurotrophic Factor and Glial Cell Line-derived Neurotrophic Factor Inhibit Ferrous Iron Influx via Divalent Metal Transporter 1 and Iron Regulatory Protein 1 Regulation in Ventral Mesencephalic Neurons
}

\author{
Ning Song ${ }^{1, \varpi, \Perp}$, Haoyun Zhang ${ }^{1,2}$, Hong Jiang ${ }^{1}$, Mingxia Bi $^{1}$ and Junxia Xie ${ }^{1}$ \\ ${ }^{1}$ Medical College of Qingdao University, China \\ ${ }^{2}$ Weifang Medical University, China
}

\begin{abstract}
Iron accumulation is observed in the substantia nigra of patients with Parkinson's disease. However, it is unknown whether neurotrophic factors, brain-derived neurotrophic factor (BDNF) and glial cell line-derived neurotrophic factor (GDNF) participate in the modulation of neuronal iron metabolism. Here, we investigated the effects and underlying mechanisms of BDNF and GDNF on the iron influx process in primary cultured ventral mesencephalic neurons. 6-hydroxydopamine-induced enhanced ferrous iron influx via improper up-regulation of divalent metal transporter 1 with iron responsive element (DMT1 + IRE) was consistently relieved by BDNF and GDNF. Both the mRNA and protein levels of DMT1+IRE were downregulated by BDNF or GDNF treatment alone. We further demonstrated the involvement of iron regulatory protein 1 (IRP1) in BDNF- and GDNF-induced DMT1+IRE expression. Extracellular-regulated kinase 1/2 (ERK1/2) and Akt were activated and participated in these processes. Inhibition of ERK1/2 and Akt phosphorylation abolished the down-regulation of IRP1 and DMT1 + IRE induced by BDNF and GDNF. Taken together, these results show that BDNF and GDNF ameliorate iron accumulation via the ERK/Akt pathway, followed by inhibition of IRP1 and DMT1 + IRE expression, which may provide new targets for the neuroprotective effects of these neurotrophic factors.
\end{abstract}

\section{Dopamine Turnover in the Nigrostriatal System of MPTP-treated Mice at the Presymptomatic and Early Symptomatic Stages of Parkinsonism}

\author{
Michael Ugrumov ${ }^{\natural, \iota, ~ A l e x a n d e r ~ K i m, ~ E l e n a ~ K o z i n a ~ a n d ~ G u l ' n a r a ~ K h a k i m o v a ~}$ \\ Institute of Developmental Biology RAS, Russia
}

\begin{abstract}
Pathogenesis of Parkinson's disease (PD) is characterized by a long-term asymptomatic development (preclinical stage) and initial appearance of motor symptoms under a threshold loss of most dopaminergic (DAergic) cell bodies in substantia nigra and axons/DA in the striatum. This study was aimed to search for compensatory mechanisms preventing motor dysfunctions at presymptomatic stage and triggers of their appearance at symptomatic stage evaluating DA turnover, synthesis (tyrosine hydroxylase (TH) mRNA, protein, activity), release (microdialysis in vivo, slices), uptake (slices), degradation (MAO mRNA, activity) at original models. At presymptomatic stage motor disorders were prevented due to plasticity of survived DAergic neurons, manifested by enhanced TH activity, increased spontaneous and stimulated release of DA, and decreased MAO-B activity. Noteworthy, striatal DA was synthesized not only in DAergic neurons but also in tandems of non-dopaminergic neurons containing individual enzymes, either TH or aromatic L-amino acid decarboxylase. At the symptomatic stage, despite maintaining of DA synthesis in DAergic neurons at the control level and an increase of DA synthesis in striatal monoenzymatic neurons, the intercellular level of DA dropped up to the threshold, inducing the appearance of motor disorders. Major triggers of motor dysfunctions were a decrease of spontaneous and stimulated release of striatal DA and an increase of MAO-A activity. The PD models of preclinical and early clinical stages, which were thoroughly specified, are valuable for a development of preventive pharmacotherapy aiming to improve compensatory processes and to inhibit triggers of motor dysfunctions for prolonging preclinical stage.
\end{abstract}




\title{
Genomics-Based Bioinformatics Analysis of Parkinson's Disease
}

\author{
$\mathrm{Li} \mathrm{M.} \mathrm{Fu}=$ \\ AHMC Healthcare, USA
}

\begin{abstract}
The pathogenesis of PD has been theorized on several biological pathways surrounding $\alpha$-synuclein, the ubiquitin-proteasome system, mitochondria, and endoplasmic reticula. We have developed a new genomics-based bioinformatics method that can discover specific upstream pathways, suggestive of disease etiology and pathogenesis, using tissue samples collected from patients and control subjects at a single point in time. We applied this method to analyze the molecular basis of Parkinson's disease (PD) pathophysiology. The results are summarized as follows. First, we confirmed the misfolded-protein theory of PD. In the upregulated genomic response, two major upstream processes identified were transcription and RNA metabolism. The increased expression of transcripts such as ATF4 and HSPA1A can be attributed to the presence of misfolded or aggregated proteins. In addition, the research results offered new Insights into the pathophysiology of PD. The finding that RNA metabolism was an upstream biological process in PD pointed to the role of RNA function in the disease's pathogenesis. Aberrant RNA function or metabolism can initiate a neurodegenerative process. This study showed that the process of "transport" was the top upstream process among the down-regulated genomic processes identified in PD. It meant that the dysfunction of the transport system led the phase of neurodegeneration when cell activity became diminished. Finally, this study answered important controversial questions concerning PD, in particular, the question of whether mitochondrial dysfunction caused neurodegeneration. We found that mitochondrial function was down-regulated and characterized as a downstream process in PD, reflecting that mitochondrial dysfunction is a weakly causal or an effect process of PD. The results of this research will have a significant impact on our current understandings of PD.
\end{abstract}

\section{The Death of Human Cortical Neurons Due to Exposure to Exogenous A $\beta$ Oligomers Prevented by NPS 2143, a Selective Antagonist of the Calcium-sensing Receptor}

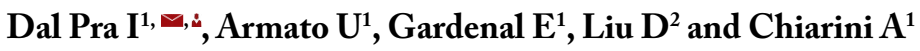 \\ ${ }^{1}$ University of Verona Medical School, Italy \\ ${ }^{2}$ Third Military Medical University, China
}

\begin{abstract}
The destructive spreading of "contagious seeds" like A $\beta 42$ and phosphorylated (p)-Tau oligomers drives the progression of Alzheimer's disease (AD). Apparently, the $A \beta 42$ oligomers (A $\beta$-os) trigger $p$-Tau oligomers ( $\mathrm{p}$-Tau-os) generation, which later will act even alone. Only neurons were held to make and spread the $\mathrm{AD}$ "seeds", astrocytes acting as scavengers and cytokine producers. However, normal functioning astrocytes from the adult human temporal cortex once exposed to exogenous soluble or fibrillar $\mathrm{A} \beta 25-35$, an $\mathrm{A} \beta 42$ proxy, make and secrete increasing loads of endogenous $\mathrm{A} \beta 42$-os, $\mathrm{p}$-Tau-os, nitric oxide (NO), and vascular endothelial growth factor (VEGF)-A causing the death of human cortical neurons. Thus, AD neuropathology slow progression is driven by self-inducing $\mathrm{A} \beta 42-\mathrm{os}$ and $\mathrm{p}$-Tau-os that recruit mounting numbers of neuron-astrocyte teams to make and secrete further amounts of the four neurotoxins. Most important, exogenous $\mathrm{A} \beta$-os specifically bind the $\mathrm{Ca} 2+-$ sensing receptors $(\mathrm{CaSRs})$ of human cortical astrocytes and neurons triggering a signaling that causes the production/secretion of surplus A 342 -os, p-Tau-os, NO, and VEGF-A. We have also proved that NPS 2143, a highly selective and specific CaSR antagonist (calcilytic), totally suppresses the $\mathrm{A} \beta / \mathrm{CaSR}$-mediated over secretion of the four mentioned $\mathrm{AD}$ neurotoxins thereby preserving the cortical neurons viability. Hence, $\mathrm{CaSR}$ antagonists (calcilytics) should be considered as anti-AD drugs as they effectively prevent the diffusion of "contagious" $\mathrm{A} \beta 42$-os and $\mathrm{p}$-Tau-os and thus the inexorable spread of cognitively impairing and ultimately deadly $\mathrm{AD}$.
\end{abstract}

\section{Synuclein Immunotherapy for Parkinson's Disease and other Synucleinopathies}

\author{
Wagner Zago ${ }^{\varpi}$ \\ Prothena Biosciences, USA
}

\section{Abstract}

Alpha-synuclein abnormally accumulates in Parkinson's disease and in other progressive degenerative diseases known as 
alpha-synucleinopathies. Scientific evidence strongly suggests that alpha-synuclein may play a central role in the pathophysiology of Parkinson's disease. Moreover, toxic alpha-synuclein species have been proposed to propagate through interconnected neurons and to initiate pathology through processes that can be intercepted by anti-alpha-synuclein antibodies. Strategies directed at targeting those toxic alpha-synuclein species have been developed in recent years and outcomes from non-clinical studies support the notion that targeting alpha-synuclein might be a viable option for the treatment of synucleionopathies. An overview of the evidence supporting cell-to-cell transmission of alpha-synuclein and the non-clinical findings that led to the development of PRX002, an anti-alpha-synuclein antibody currently in clinical studies for Parkinson's disease, will be presented.

\title{
Role of GRKS in L-DOPA-induced Dyskinesia
}

\author{
Eugenia V. Gurevich ${ }^{\varpi, \varkappa}$, M. Rafiuddin Ahmed and Vsevolod V. Gurevich \\ Vanderbilt University Medical Center, USA
}

\begin{abstract}
Parkinson's disease is caused by degeneration of dopaminergic neurons and the consequent deficit of dopamine in the striatum. Therapy with the dopamine precursor L-DOPA is the mainstay of treatment. However, patients develop L-DOPA-induced dyskinesia (LID), likely due to excessive signaling via dopamine receptors. G protein-coupled receptor kinases (GRKs) control desensitization of dopamine receptors. Here we demonstrate that LID is attenuated by lentivirus-mediated overexpression of GRK6 in the striatum in rodent and primate models of Parkinson's disease. Conversely, GRK6 knockdown exacerbated LID in parkinsonian rats. GRK6 suppressed dyskinesia in monkeys without compromising the antiparkinsonian effects of L-DOPA and even prolonged the antiparkinsonian effect of a lower dose. Another GRK isoform expressed by striatal neurons, GRK3, also attenuated LID when overexpressed in the striatum, whereas knockdown of GRK3 exacerbated it. In contrast with GRK6, which needs to be catalytically active to have the anti-LID effect, kinase-dead GRK3 and its separated RGS homology domain (RH) suppressed LID, whereas GRK3 w ith disabled RH did not. RH alleviated LID without compromising anti-akinetic effect of L-DOPA. RH binds active Gq. Both GRK6 and GRK3 inhibited accumulation of $\triangle$ FosB, a marker of LID, with the effect of GRK3 on $\triangle$ FosB being independent of its kinase activity. Our finding that increased availability of GRK6 and GRK3 ameliorates LID. Our results also reveal a novel mechanism of GRK3 control of the dopamine receptor signaling and the role of $\mathrm{Gq}$ in LID. Manipulation of GRK function in the striatum offers hope of controlling both LID and motor fluctuations in Parkinson's disease.
\end{abstract}

\section{A Case of Anoxic Brain Damage-an Anthropological Perspective}

\author{
Elisabeth L'orange Furst ${ }^{\square}$ \\ University of Oslo, Norway
}

\begin{abstract}
My focus is a serious case of anoxic brain damage, the result of a cardiac arrest that suddenly struck a 53 years old man and lasted 3/4 of an hour. He was cooled down and brought into artificial coma for ten days, then he had a rapid recovery and soon was on his feet. However his cognitive abilities were damaged. His orientation of time and place were lost, his ability to see was impaired. After some years he was diagnosed by dementia in need of help all the 24 hours. This man is my husband. What happened to him turned me, a researcher in anthropology, into a next of kin, a care-performing wife. The situation was shocking, but also so intriguing that I soon found myself, trained as I am in the methodology of long term participant observation, observing his condition, raising research questions and writing about it. Bringing him home after 8 months in hospitals, taking care of him together with personal assistants, implied close observations and interactions that taught us how to communicate with him to make him able to manage the basic challenges of everyday life. My experiences in the double role of a next of kin and a scholar has convinced me of the importance of bringing humanistic perspectives into the fields of medicine. My observations and research questions are mainly influenced by the philosophy of phenomenology, especially by Maurice Merleau-Ponty and his concept of the 'body subject' that transcends Western medicine's dualism of body and soul.
\end{abstract}




\title{
Ganglioside GM1 Deficiency as a Cause of Parkinson's Disease
}

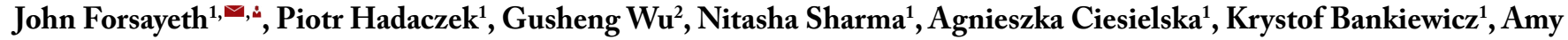 \\ L. Davidow ${ }^{3}$, Lu Zi-Hua ${ }^{2}$ and Robert W. Ledeen ${ }^{2}$ \\ ${ }^{1}$ Department of Neurological Surgery, University of California San Francisco, USA \\ ${ }^{2}$ Department of Neurology and Neurosciences MSB-H506, New Jersey Medical School, Rutgers, The State University of New Jersey, USA \\ ${ }^{3}$ Department of Biostatistics/Epidemiology, New Jersey Medical School, Rutgers, The State University of Nerw Jersey, USA
}

\begin{abstract}
GDNF is indispensable for adult catecholaminergic neuron development, and failure of GDNF signaling has been linked to loss of dopaminergic neurons in Parkinson's disease (PD). The present study demonstrates strongly attenuated GDNF signaling in neurons deficient in ganglio-series gangliosides, and restoration of such signaling with LIGA20, a membrane permeable analog of GM1. GM1 is shown to associate in situ with GFR $\alpha 1$ and RET, the protein components of the GDNF receptor, this being necessary for assembly of the tripartite receptor complex. Mice wholly or partially deficient in GM1 developed PD symptoms based on behavioral and neuropathological criteria which were largely ameliorated by gene therapy with AAV2GDNF and also with LIGA20 treatment. Nigral neurons of PD subjects that were severely deficient in GM1 showed subnormal levels of tyrosine phosphorylated RET. Also in PD brain, GM1 levels in the occipital cortex, a region of limited PD pathology, were significantly below age-matched controls, suggesting the possibility of systemic GM1 deficiency as a risk factor in PD. Together with age-related decline of brain GM1, this points to gradual development of subnormal GM1 in brain of PD subjects below levels required for effective GDNF signaling. This hypothesis offers a dramatically different explanation for the etiology of sporadic PD as a manifestation of acquired resistance to GDNF.
\end{abstract}

\section{Saposin C and the Glucocerebrosidase/Alpha-synuclein Connection in Parkinson's Disease}

\author{
James M. Gruschus ${ }^{1, \varpi, \varkappa}$, Zhiping Jiang ${ }^{1}$, Thai Leong Yap ${ }^{1}$, Stephanie A. Hill1, Jennifer C. Lee ${ }^{1}$, Alexander Grishaev ${ }^{2}$, \\ Grzegorz Piszczek ${ }^{3}$ and Ellen Sidransky ${ }^{4}$
}

${ }^{1}$ Laboratory of Molecular Biophysics, National Institutes of Health, USA

${ }^{2}$ Laboratory of Chemical Physics, National Institute of Diabetes and Digestive and Kidney Diseases, National Institutes of Health, USA ${ }^{3}$ Biophysics Core, Biochemistry and Biophysics Center, National Heart, Lung, and Blood Institute, National Institutes of Health, USA

${ }^{4}$ Section on Molecular Neurogenetics, Medical Genetics Branch, National Human Genome Research Institute, National Institutes of Health, USA

\begin{abstract}
Mutations in the gene for the lysosomal enzyme glucocerebrosidase (GCase) cause Gaucher disease and are the most common risk factor for Parkinson's disease (PD). Analytical ultracentrifugation of $8 \mu \mathrm{M}$ GCase shows equilibrium between monomer and dimer forms. However, in the presence of its co-factor saposin C (Sap C), only monomer GCase is seen. Isothermal calorimetry confirms that $\mathrm{Sap} C$ associates with GCase in solution in a 1:1 complex $(\mathrm{Kd}=2.1 \pm 1.1 \mu \mathrm{M})$. Saturation cross-transfer NMR determined that the region of Sap C contacting GCase includes residues 63-66 and 74-76, which is distinct from the region known to enhance GCase activity. Because $\alpha$-synuclein ( $\alpha$-syn), a protein closely associated with PD etiology, competes with Sap C for GCase binding, its interaction with GCase was also measured by ultracentrifugation and saturation cross-transfer. Unlike Sap C, binding of $\alpha$-syn to GCase does not affect multimerization. However, adding $\alpha$-syn reduces saturation cross-transfer from Sap C to GCase, confirming displacement. To explore where Sap C might disrupt multimeric GCase, GCase x-ray structures were analyzed using the program PISA, which predicted stable dimer and tetramer forms. For the most frequently predicted multimer interface, the GCase active sites are partially buried, suggesting that Sap C might disrupt the multimer by binding near the active site.
\end{abstract}

\section{Tremor in Parkinson's Patients Using Deep Brain Stimulation and Cueing}

\author{
Tjitske Heida T ${ }^{1, \bullet, \AA}$, Yan $Z^{1}{ }^{1}$ and Richard van Wezel ${ }^{1,2}$ \\ ${ }^{1}$ MIRA Institute, University of Twente, The Netherlands \\ ${ }^{2}$ Donders Institute, The Netherlands
}




\begin{abstract}
Rest tremor is the most common and easily recognized symptom of Parkinson's disease (PD), but many questions still exist with regard to its origin and the neuronal pathways involved. The occurrence of action tremor is less well recognized in PD, but often more disabling. The cerebello-thalamocortical loop has been suggested to indirectly contribute to the expression of parkinsonian tremor. The observed overactivation in cerebellar loops has also been associated with a higher ability in PD patients to perform repetitive movements that are cued by auditory or visual stimuli. Deep brain stimulation (DBS) is currently an accepted treatment for advanced PD. DBS operates with different clinical efficacy based on the specific motor deficit, which may also be task-specific. While DBS directly influences neuronal activity patterns in the basal ganglia, it may be expected that modulation of the cerebellar loops may have an additional effect on parkinsonian tremor if both loops are involved in tremor generation and expression. We explored whether rest and action tremor react differently to clinically effective and less effective DBS using quantitative tremor analysis methods. In addition, we have tested whether the combination of DBS and auditory cueing has an enhanced effect on action tremor reduction. Compared to the resting condition and the performance of self-paced hand or foot movements, the number of extremities showing tremor was significantly reduced under external cueing conditions when stimulation was "on". With DBS “off", only the lower cueing frequencies $(1.6$ and $3.2 \mathrm{~Hz}$ ) provided a beneficial effect.
\end{abstract}

\title{
Progress in Development of Anti-parkinson Drug with Unique Specificity
}

\author{
Judit Ovadi ${ }^{-}$ \\ Hungarian Academy of Sciences, Hungary
}

\begin{abstract}
Neomorohic Moonlighting Proteins perform distinct functions at physiological and pathological conditions without alterations of gene level; their prototype is the disordered Tubulin Polymerization Promoting Protein (TPPP/p25) which modulates the dynamics and stability of the microtubule system via its bundling and tubulin acetylation promoting activities. These physiological functions are mediated its direct associations with tubulin/microtubules as well as tubulin deacethylases, HDA6 and SIRT2. In normal brain TPPP/p25 by expressing oligodendrocytes plays crucial role in the formation of projections on the course of differentiation requested for the axon ensheathment. Slicing of TPPP/p25 by siRNA or miR206 impedes the differentiation of oligodendrocytes favoring cell proliferation. TPPP/p25 does not have a well-defined 3D structure; it is an intrinsically disordered protein involved in the etiology of distinct CNS diseases: multiple sclerosis (demyelination disease), oligodendroglioma (tumor) or synucleinopathies. In the last case TPPP/p25 forms pathological complex with $\alpha$-synuclein, leading to the formation of inclusions such as Lewy body. The co-enrichments and co-localization of $\alpha$-synuclein and TPPP/p25 were visualized by confocal fluorescence microscopy in brain characteristic for Parkinson's disease and multiple system atrophy, in spite of the fact, that in normal brain these two hallmark proteins are expressed distinctly in neuron and oligodendrocytes, respectively. The binding segment on TPPP/p25 involved in the physiological and pathological interactions has been identified at molecular and cell levels. The dissimilarity of these binding motives responsible for the neomorphic moonlighting feature of TPPP/p25 has significant innovative impact in anti-Parkinson drug research.
\end{abstract}

\section{Mitochondrial Fission at the Nerve Terminal in Susceptible Neurons}

\author{
Ken Nakamura ${ }^{1,2, \square, 2,}$, Amandine Berthet ${ }^{1}$, Lauren Y. Shields ${ }^{1,2}$, Hwajin Kim ${ }^{1}$, Dominik Haddad ${ }^{1}$, Elyssa B. Margolis ${ }^{2}$, \\ Lei Zhu ${ }^{1}$, Hiromi Sesaki ${ }^{3}$, Lennart Mucke ${ }^{1,2}$ and Eric J. Huang ${ }^{2}$
}

${ }^{1}$ Gladstone Institute of Neurological Disease, USA

${ }^{2}$ University of California, San Francisco, USA

${ }^{3}$ Johns Hopkins University, USA

\begin{abstract}
Changes in the activity of the central mitochondrial fission protein dynamin-related protein 1 (Drp1) may contribute to the pathophysiology of several neurologic diseases. However, the normal functions of mitochondrial fission in neurons are poorly understood, which limits our understanding of the nature and significance of mitochondrial changes in disease. To gain insight into the normal roles of mitochondrial fission in susceptible populations of neurons, we created mouse models in which Drp1 has been deleted in either midbrain dopamine (DA) neurons, or in CA1 and other forebrain neurons. We found that both neuronal populations require mitochondrial fission for normal function; however, DA neurons in the substantia nigra, and their projections, are uniquely susceptible to Drp1 loss, likely, in part, because they require Drp1 to target mitochondria to their axons. Indeed, we show that neurons require mitochondrial fission to maintain normal levels of mitochondrial-derived ATP at the
\end{abstract}


nerve terminal, but not at the cell body. These bioenergetic deficits occurred equally in boutons with and without mitochondria, indicating that ATP is rapidly dispersed in axons and that the deficits in axonal bioenergetics are not caused by regional energy gradients. Instead, our findings suggest that loss of Drp1 compromises the intrinsic bioenergetic function of axonal mitochondria, revealing a mechanism by which disrupting mitochondrial dynamics can cause selective dysfunction of axons.

\title{
Sulfanylphthalimides: A Novel Class of Dual-acting Compounds for the Treatment of Parkinson's and Alzheimer's Disease
}

\author{
Mietha M. Van der Walt ${ }^{1, \varpi, 4}$, Gisella Terre'Blanche ${ }^{1}$, Anel Petzer ${ }^{2}$ and Jacobus P. Petzer ${ }^{1}$ \\ ${ }^{1}$ Pharmaceutical Chemistry, School of Pharmacy, North-West University, South Africa \\ ${ }^{2}$ Centre of Excellence for Pharmaceutical Sciences, North-West University, South Africa
}

\begin{abstract}
In complex neurological diseases, such as Parkinson's disease (PD) and Alzheimer's disease (AD), recent advances suggest that dual-target directed drugs may be advantageous over single-target treatments. The adenosine antagonists (A1 and A2A subtypes) and monoamine oxidase (MAO) B selective inhibitors are considered to be important targets for the development of new therapies for PD and AD. Such dual-target directed compounds may act synergistic in the treatment of PD: antagonism of the $\mathrm{A} 1$ and $\mathrm{A} 2 \mathrm{~A}$ receptors may facilitate dopamine release and signaling, while MAO-B inhibition may reduce the central catabolism of dopamine. Additionally, it may be reasoned that dual-target directed compounds may find therapeutic value in the treatment of $\mathrm{AD}$ : antagonism of the $\mathrm{A} 1$ receptor may be beneficial in the treatment of cognitive dysfunction, while antagonism of the A2A receptor and MAO-B inhibition may exhibit neuroprotective effects. The present work represents the first extensive report on the novel sulfanylphthalimide analogues, a previously unknown non-xanthine class of adenosine receptor antagonists and MAO-B inhibitors. The results document that the sulfanylphthalimide analogues are selective for the adenosine $\mathrm{A} 1$ receptor over the $\mathrm{A} 2 \mathrm{~A}$ receptor subtype, with a number of compounds also possessing $\mathrm{MAO}-\mathrm{B}$ inhibitory properties. Among the compounds evaluated, 5-[(4-methoxybenzyl) sulfanyl]phthalimide was found to possess the highest binding affinity to adenosine A1 receptors with a $\mathrm{Ki}$ value of $0.369 \mu \mathrm{M}$. This compound is reported to also inhibit MAO-B with an IC50 value of $0.020 \mu \mathrm{M}$. In conclusion, the sulfanylphthalimides may serve as lead compounds for the design of nonxanthine dual-target directed compounds for treating neurological disorders.
\end{abstract}

\section{Clinical Features and Diagnosis of Chronic Traumatic Encephalopathy}

\author{
Philip Montenigro ${ }^{1,2}$ \\ ${ }^{1}$ Boston University School of Medicine, USA \\ ${ }^{2}$ Chronic Traumatic Encephalopathy Center, USA
}

\begin{abstract}
Chronic traumatic encephalopathy (CTE) is a neurodegenerative disease associated with repetitive brain-injury. Despite sharing similarities with other diseases, like Alzheimer's disease, CTE is distinct both pathologically and clinically. The clinical features of CTE are often progressive, with dramatic changes in mood, behavior, and cognition, often resulting in dementia. Some cases develop motor impairments, such as Parkinsonism. To date, the only definitive means to diagnose CTE is through post-mortem autopsy. However, the ability to diagnose CTE during life is necessary to develop epidemiologic investigations, identify risk factors, and initiate treatment trials. To address this gap, new clinical research diagnostic criteria for CTE have been proposed (Montenigro 2014). Efforts to validate the utility of the proposed criteria are currently underway. In this presentation an overview of the history and current state of knowledge of CTE will be provided. First, the historical origins of CTE will be revealed and the earliest reported case-studies summarized. Next, a review of neuropathological investigations and recent research into the clinical manifestations of CTE will be considered. Lastly, new clinical diagnostic criteria for CTE will be summarized and preliminary findings from ongoing investigations discussed.
\end{abstract}




\title{
The Role of SNCA Gene in Lewy Body Spectrum Disorders: Genetic Variations, Expression and Splicing
}

\author{
Ornit Chiba-Falek ${ }^{1, \varpi, \dot{ }}$, Michae ${ }^{1}$ W. Lutz ${ }^{1}$, Robert Saul ${ }^{2}$ and Allen D. Roses ${ }^{1,3}$ \\ ${ }^{1}$ Duke University School of Medicine, USA \\ ${ }^{2}$ Polymorphic DNA Technologies, USA \\ ${ }^{3}$ Zinfandel Pharmaceuticals Inc., USA
}

\begin{abstract}
Synucleinopathies are a group of neurodegenerative disorders that share a common neuropathological characteristic composed of intracellular protein aggregates, known as Lewy bodies (LB), in affected brains. The LBs consist largely of alphasynuclein (SNCA) protein. Our bypothesis is that the genetic variants reported to be associated with complex neurodegenerative diseases, including Parkinson's disease (PD) and dementia with LB (DLB), modulate directly, or tag causal variants that modulate the expression of critical disease genes, and that altered gene expression is a major determining mechanism of complex disease susceptibility. SNCA gene has been identified as a highly significant genetic risk factor for PD and DLB, however the precise genetic variants within $S N C A$ locus underlying the observed genetic association, and the functional effects these variants exert to promote disease susceptibility, remain largely undescribed. The talk will present advances in filling in these gaps and will focuses on the role of the $S N C A$ gene in LB spectrum disorders, including LB variant of Alzheimer's disease (LBvAD) and DLB. Our team embarked on an effort to identify non-coding structural variants (e.g. STR, SSR and indel) within $S N C A$ and explore their functional consequences in a broad spectrum of disorders that exhibit LB pathology. Using a bioinformatic algorithm followed by deep sequencing analyses we have recently identified genetic variants that are associated with LB risk and with alterations in SNCA expression profile. These findings may have future implications for drug discovery and development of genetic-biomarker signatures.
\end{abstract}

\section{The Role of Sirtuins in Alzheimer's Disease: A New Therapeutic Target}

\author{
Y1l1z Dincer ${ }^{\varpi}$ \\ Istanbul University, Turkey
}

\begin{abstract}
Alzheimer's Disease (AD) is a neurodegenerative disorder characterized by neurological dysfunction resulting in memory loss, cognitive and functional decline. The hallmarks of disease are the accumulation of $\beta$-amyloid (A $\beta$ ) neuritic plaques and neurofibrillary tangles formed by hyperphosphorylated tau protein. AD is a very common disorder and advancing age of the global population dramatically increases the incidence of $\mathrm{AD}$. The present therapies are symptomatic and do not alter disease progression. Researches have focused on noval, effective therapies. Sirtuins are a family of nicotinamide adenine dinucleotide (NAD+)-dependent protein deacetylases. They play regulator role in multiple pathways such as mitochondrial energetic, cellcycle regulation, oxidative stress resistance, insulin secretion and inflammation. Seven sirtuins (SIRT1-7) were described in humans. Each enzyme has a distinct subcellular localization, substrate specificity and biological activity. SIRT1 and SIRT2 are the sirtuins that have been extensively studied in AD. Most of the recent studies point to opposing effects of SIRT1 and SIRT2: SIRT1 up-regulation is protective, SIRT2 activation is toxic to neuronal cells. SIRT1 has been shown to suppress AD by reducing $A \beta$ production in cells, primary neurons, and mouse models. SIRT1 deficiency has been shown to lead to accumulation of phosphorylated tau and $\mathrm{A} \beta$ in mouse models of $\mathrm{AD}$ and in $\mathrm{AD}$ patients. SIRT2 inhibition has been shown to reduce hyperphosphorylated tau and to restore cognition in AD transgenic mice. Therapies that are targeted towards modulation of SIRT1 and SIRT 2 activities may be effective in slowing down or preventing neurodegeneration and subsequent cognitive impairment observed in AD.
\end{abstract}

\section{Cognitive Rehabilitation in Alzheimer's Disease: Concepts, Evidence and Challenges}

\author{
Martine Simard ${ }^{1,2, \varpi,\llcorner}$, Laurence Brunelle-Hamann ${ }^{1,2}$ and Stéphanie Thivierge ${ }^{1,2}$ \\ ${ }^{1}$ School of Psychology, University Laval, Canada \\ ${ }^{2}$ Institut universitaire en santé mentale de Québec, Canada
}




\begin{abstract}
The literature on Alzheimer's disease (AD) describes three types of cognitive interventions in addition to reality orientation therapy: cognitive stimulation, cognitive training and cognitive rehabilitation. Cognitive stimulation involves a set of group activities and discussions, and targets general cognitive and social functioning. Cognitive training addresses specific aspects of cognition using guided practice on standardised tasks. Cognitive rehabilitation targets specific functional difficulties relevant to everyday life in individually-tailored interventions using cognitive techniques. Cochrane reviews have reported insufficient evidence to support cognitive training in $\mathrm{AD}$. Cognitive stimulation, however, has been found to improve cognition as measured by short cognitive tests, but not to ameliorate instrumental activities of daily living (IADL). According to the Adaptive Control of Thoughts model (Anderson, 1982), executive functions, episodic and working memory are involved in the first phases of procedural learning that underlie IADL. This model thus supports the utilization of memory training techniques for rehabilitation of IADL. The presentation will illustrate this point using the results of a block-randomized cross-over controlled study that has assessed the effectiveness of a cognitive rehabilitation program using two episodic memory techniques to relearn forgotten IADL in $20 \mathrm{AD}$ patients. The trained group, compared with the untrained group, has significantly improved its performance on the direct measure of training (IADL). Improvements were maintained during 3 months. Furthermore, patients registered a reduction of delusions during the second half of the study, whereas aberrant motor behaviors increased more in the treatment condition than in the control condition. Limitations and future directions of cognitive rehabilitation in $\mathrm{AD}$ will be discussed.
\end{abstract}

\title{
Impact of APOE $\& 4$ on Medial Temporal Lobe Structures, Implications for Prevention of Alzheimer's Disease
}

\author{
Joy L. Taylor ${ }^{1,2, \varpi, ょ, ~ B l a k e ~ K . ~ S c a n l o n ~}{ }^{1,2}$, Michelle Farrel1 ${ }^{1,2}$, Beatriz Hernandez ${ }^{1,2}$, Maheen M. Adamson ${ }^{1,2}$, J. Wesson \\ Ashford ${ }^{1,2}$, Greer M. Murphy Jr. ${ }^{2}$ and Michael W. Weiner ${ }^{3,4}$ \\ ${ }^{1}$ Veterans Affairs Palo Alto Health Care System, USA \\ ${ }^{2}$ Stanford University School of Medicine, USA \\ ${ }^{3}$ San Francisco VA Medical Center, USA \\ ${ }^{4}$ University of California-San Francisco, USA
}

\begin{abstract}
After age, $\mathrm{APOE} \varepsilon 4$ is the major risk factor for late onset Alzheimer's disease (AD). APOE $\varepsilon 4$ increases $\mathrm{AD}$ risk by lowering age of symptom onset, but the mechanisms are unclear. APOE $\varepsilon 4$ carriers tend to accumulate cerebral amyloid (A $\beta)$ at an earlier age, consistent with slower clearance of A $\beta$. APOE $\varepsilon 4$ has also been linked to impaired neurogenesis. Consequently, there is growing interest in how APOE $\varepsilon 4$ influences brain development, particularly in AD-vulnerable brain regions such as the hippocampus. Interestingly, evidence for structural differences related to APOE $\varepsilon 4$ have been reported in infants and adolescents. Given the considerable evidence suggesting that APOE $\varepsilon 4$ affects both early brain development and A $\beta$-mediated risk of $\mathrm{AD}$, we performed a longitudinal MRI study involving cognitively normal adults of a wide age range. Subjects (25 APOE $\varepsilon 4$ carriers and $31 \varepsilon 3 / \varepsilon 3$ homozygotes) were initially aged $51-75$ years. We used a growth curve approach, which can provide information on $\varepsilon 4$-related differences initially and later in life. Longitudinally measured hippocampal volume was the primary outcome; nearby medial temporal regions were secondary outcomes. APOE $\& 4$ carriers had significantly smaller initial hippocampal volumes than $\varepsilon 3$ homozygotes. Rate of hippocampal atrophy was not greater in the APOE $\varepsilon 4$ group, even though age-related atrophy was detected in the overall sample. The longitudinal results add to the growing evidence that APOE $\varepsilon 4$ is associated with smaller hippocampal size early in life. Findings will be discussed in terms of Alzheimer's disease prevention and treatment strategies.
\end{abstract}

\section{Modulators of Proteasome Function and $\beta$-amyloid Toxicity}

\author{
Wail M. Hassan ${ }^{\square,\llcorner}$, Brandon Schmitt and Aaron B. Taylor \\ University of Wisconsin - Milwaukee, USA
}

\begin{abstract}
$\beta$-amyloid $(\mathrm{A} \beta)$ plays a central role in the pathogenesis of Alzheimer's disease (AD). Enhanced $A \beta$ production and impaired clearance are associated with increased AD risk. Degradation by the proteasome is one of the main mechanisms of intracellular A $\beta$ clearance. We have previously shown that AIP-1 reduces A $\beta$ toxicity and accumulation in a nematode model of Alzheimer's disease. In this model, AIP-1 is induced in the tissues of Caenorbabditis elegans in response to transgenic expression of human
\end{abstract}


$\mathrm{A} \beta$. This protein, as well as its mammalian homologues, has been shown to associate with the 19S regulatory subunit of the proteasome and play a role in modulating proteasomal function. There are two mammalian homologues of AIP-1, AIRAP and AIRAPL. We were interested to know whether either or both of these proteins were protective against $A \beta$ toxicity. To test this hypothesis, we overexpressed each protein individually in A $\beta$-expressing worms and showed that AIRAPL, but not AIRAP, exhibited a protective effect similar to that of AIP-1. Ongoing research addresses the putative protective role of AIP-1 mammalian homologues in mammalian tissue culture cells. In this presentation, I will summarize our most recent data in this line of research.

\title{
Granulovacuolar Degeneration and Tau Fibrillary Pathology
}

\author{
Christoph Kohler ${ }^{\square, \imath}$ and Maja Dinekov \\ Institute II for Anatomy, University of Cologne, Germany
}

\begin{abstract}
Granulovacuolar degeneration (GVD) is a neurodegenerative change that appears as granule-containing vacuoles in the cytoplasm of neurons. In addition to extracellular senile plaques and intraneuronal neurofibrillary tangles (NFTs) that consist of abnormal fibrils of the microtubule-associated protein tau, severe GVD in the hippocampus is a histopathological hallmark of Alzheimer's disease (AD). A staging system for GVD has recently been established. Granulovacuolar inclusions are present in neurons together with hyperphosphorylated tau and can be labeled with markers of the activated unfolded protein response (UPR), a stress response that becomes activated by the accumulation of unfolded proteins in the endoplasmic reticulum. We studied the presence of GVD and the UPR in transgenic $\mathrm{pR} 5$ mice that express the longest human tau isoform together with the pathogenic mutation P301L and in APPSLxPS1mut mice that develop an A $\beta$ plaque pathology. Tau was hyperphosphorylated in many neurons already in young pR5 mice. A subset of these neurons developed an advanced stage of tau hyperphosphorylation and early tau fibrillary pathology. These neurons displayed GVD, but we rarely saw GVD in neurons with mature NFTs or early tau hyperphosphorylation. We did not find neurons with GVD in 20 months-old APPSLxPS1mut mice and only exceptionally in very old non-transgenic mice. The granulovacuolar inclusions in pR5 mice were labeled with markers of the activated UPR, similar to what has been observed by others in studies on human autopsy cases. These findings suggest that GVD and activation of the UPR accompany early stages of NFT formation in pR5 mice.
\end{abstract}

\section{Neurotensin and Alzheimer's Disease: Therapeutic Potential of NTS1 Agonists}

\author{
Saobo Lei \\ University of North Dakota, USA
}

\begin{abstract}
Neurotensin (NT) is a 13-amino-acid peptide distributed in the brain including the entorhinal cortex (EC), a structure that is crucial for learning and memory and undergoes the earliest pathological alterations in Alzheimer's disease (AD). Although NT is implicated in modulating cognition, the cellular and molecular mechanisms whereby NT modifies cognitive processes and the potential therapeutic roles of NT in AD are unknown. We studied the effects of NT on neuronal excitability and spatial learning in the EC where high density of NT receptors is expressed. Brief application of NT induced persistent increases in action potential firing frequency which could last for at least 1h. NT-induced facilitation of neuronal excitability was mediated by down-regulation of TREK-2 K+ channels and required the functions of NTS1, phospholipase C and protein kinase C. Microinjection of NT or NTS1 agonist, PD149163, into the EC increased spatial learning as assessed by the Barnes Maze Test. Activation of NTS1 receptors also induced persistent increases in action potential firing frequency and significantly improved the memory status in APP/PS1 mice, an animal model of AD. Our study identifies a cellular substrate underlying learning and memory and suggests that NTS1 agonists may exert beneficial actions in an animal model of AD.
\end{abstract}

\section{Proteolytic Cleavage of Apolipoprotein E4 as the Keystone for the Heightened Risk Associated with Alzheimer's Disease}

\author{
Troy T. Rohn ${ }^{\square, ょ, ~ J u l i a ~ E . ~ L o v e ~ a n d ~ R y a n ~ J . ~ D a y ~}$
}

Boise State University, USA 


\begin{abstract}
Alzheimer's disease $(\mathrm{AD})$ is a progressive neurodegenerative disease characterized by microscopic lesions consisting of beta-amyloid plaques and neurofibrillary tangles (NFTs). The majority of cases are defined as sporadic and are likely caused by a combination of both genetic and environmental factors. Of the genetic risk factors identified, the $34 \mathrm{kDa}$ protein, apolipoprotein (apo) E4, is of significance importance as $A P O E 4$ carriers account for $65-80 \%$ of all $\mathrm{AD}$ cases. Although apoE4 plays a normal role in lipoprotein transport, how it contributes to $\mathrm{AD}$ pathogenesis is currently unknown. One potential mechanism by which apoE4 contributes to disease risk is its propensity to undergo proteolytic cleavage generating $\mathrm{N}$ - and $\mathrm{C}$-terminal fragments. In this presentation, we will highlight our recent results supporting a role of apoE4 proteolysis as a potential mechanism leading to $\mathrm{AD}$ risk. Data will also presented examining apoE proteolysis in other neurodegenerative diseases including vascular dementia and Pick's disease.
\end{abstract}

\title{
A Potential Function of Secreted Exosomes in Sequestering Alzheimer's Amyloid- $\beta$ Peptide
}

\author{
Yasuyuki Igarashi ${ }^{\square, \imath}$ and Kohei Yuyama \\ Hokkaido University, Japan
}

\begin{abstract}
Increased amyloid- $\beta$ peptide $(A \beta)$ in brain is linked to the pathogenesis of Alzheimer's disease (AD). Exosome, an extracellular lipid vesicle, contains several proteins related to neurodegenerative disorders, including $A \beta$. However, the role of the exosomes in $\mathrm{AD}$ pathogenesis is largely unknown. In this study, we examined the effects of the exosomes on $\mathrm{A} \beta$ binding and clearance in $\mathrm{AD}$ pathogenesis. We collected the exosomes from culture medium of $\mathrm{N} 2 \mathrm{a}$ cells or mouse primary cortical neurons and used them for both in vitro and in vivo studies. For exosome infusion study, transgenic mice expressing the human APP (amyloid precursor protein) bearing familial AD mutations were used. We intracerebrally infused the exosomes continuously for two weeks. We demonstrated that the exosomes were abundant in glycosphingolipid (GSLs) and coupled with A $\beta$ in a GSLdependent manner. The exosome-bound $A \beta$ was then incorporated into brain-resident phagocytes, microglia, for degradation, suggesting that the exosomes contribute to $A \beta$ clearance. Moreover, we found that the exosome production was modulated by sphingolipid metabolism. Inhibition of sphingomyelin synthase activity enhanced exosome secretion and accelerated $A \beta$ clearance in a transwell study. Consistent with the notion of in vitro study, administration of the exosomes into mouse brains resulted in marked reductions in $\mathrm{A} \beta$ levels, amyloid depositions, and $\mathrm{A} \beta$-mediated synaptotoxicity in $\mathrm{AD}$ model mice. Our data revealed that the exosomes act as potent scavengers for $A \beta$. Improving $A \beta$ clearance by upregulation of endogenous exosome secretion would be a novel strategy for AD therapy.
\end{abstract}

\section{Insulin Degrading Enzyme and Alzheimer Disease and Type 2 diabetes}

\author{
Hugo Gutierrez-Hermosillo ${ }^{1,2, \varpi, \varkappa}$ and Enrique Diaz de Leon-Gonzalez ${ }^{3}$ \\ ${ }^{1}$ UMAE No.1 CMN Bajio, IMSS, Mexico \\ ${ }^{2}$ Hospital Aranda de la Parra, Mexico \\ ${ }^{3}$ IMSS, Mexico
}

\begin{abstract}
On the last few decades we have witnessed an interesting transformation on the population pyramids throughout the world, in the USA it is expected that by the year 2020 there will be a $16 \%$ in prevalence of AD among those older than 65 years. It is know that diabetic patients have an increased risk for $\mathrm{AD}$, not completely understood the association: Therefore we carried out a case control study to evaluate the association between these 2 diseases. Our general objective was to determine the association between Alzheimer disease in diabetic patients and the $\mathrm{C}$ allele of the insulin degrading enzyme (IDE), in Mexican patients. 97 patients were included, 50 belonged to the case group and 47 to the control group. Regarding the physical examination and the results of labs there were no differences among the two groups. We found a $98 \%$ of prevalence of the IDE among the sample studied, with a well defined difference among the two groups studied. After analyzing the results we found an association between homozygote's for the CC genotype and dementia with an estimated risk of OR 2.5 on the bivariate test, and on the multivariate the OR was estimated 3.3. There was an under expression of the APOE allele. Therefore we concluded that the evidence has shown an association between the $\mathrm{C}$ allele of the IDE and cognitive impairment, in the studied population, even after controlling the confounders.
\end{abstract}




\title{
Rationale and Discussion into Parallels on Depression and Alzheimer's Disease and Different Genomic Vulnerability Leading to Therapeutic Utilities
}

\author{
Roberto Rodrigues ${ }^{\square,\llcorner}$, George Perry and Robert Petersen \\ University of Texas San Antonio, USA
}

\begin{abstract}
This presentation is a brief report of the conclusions in which we have engaged from considering that Alzheimer's disease, cognitive impairment and aging can progress with analogous molecular and/or clinical signaling/phenotypes, with no frontiers between their genotypes or phenotype. We have also proposed that chronic depression, with symptoms or not of anxiety/stress, may interact with these cognitive manifestations - already product of genomic vulnerability - at any stage of the disease, once there is also a genomic susceptibility related to a series of genes in depression and mutants with no clear identification until now. Two main notions have helped us to arrive at those studies and proposals. The first one consists of simple statistical observation of the progressive incidence of Alzheimer's disease on the normal population from the age of 65 to $100+$ years when incidence doubles every 5-10 years. We have observed that in a simulated closed cohort of normal elders - i.e. no other individual enters, no one from the cohort dies till 100+ years of age - the statistical count on this view is less confounding than the usual one, where many individuals die during the progress of disease. In conclusion, we would find that from such individuals in the hypothetical cohort only $17-20 \%$ would be normal at 100 years of age under traditional diagnostic parameters. Moreover, about $80 \%$ of the subjects consequently could be diagnosed as $\mathrm{AD}$ carriers, suggesting that Alzheimer's disease may be a kind of normality of aging - senility of AD type (SAT), 80\% - and, inversely, normality of aging would be the exception or four times less than AD - senility of no Alzheimer type(SNAT), 20\%. The second notion implies that clinical observation of both Alzheimer's disease and Depression may evidence a "spectrum" of symptoms. As some studies have shown, this spectrum initiates on normal individuals by way of absent or very mild and bearable syndromes; in other subjects the incidence of more penetrating symptoms raise leading finally to disease; and some others go to the AD last phases or are Major Depression carriers. Next, we present also some main molecular signaling phenotypes in these two illnesses and show an interacted neurochemical processing on brain areas. We suggest that the macromolecular reactions in Depression constitute a "transversal cross-talking signaling"; and in AD "dynamic or longitudinal cross-talking signaling”; both interact, particularly through oxidative stress. We conclude that the unique difference between the two pathologies may be genomic vulnerability briefly address about some future derivative neuropsychiatric treatments to be studied.
\end{abstract}

\section{Effects of Natural Cerebrolysin on the Early Intervention of Alzheimer's Disease and its Molecular Mechanism}

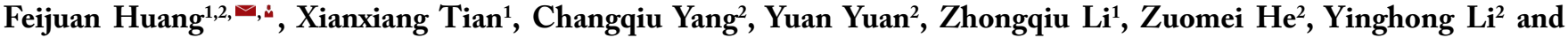 \\ Zhengzhi Wu ${ }^{1,2}$ \\ ${ }^{1}$ Shenzhen Institute of Gerontology, China \\ ${ }^{2}$ Shenzhen Institute of Integrated Chinese and Western Medicine, China
}

\begin{abstract}
Alzheimer disorder $(\mathrm{AD})$, a progressive neurodegenerative disorder mainly characterized by cognitive decline and memory deficits, is the most common cause of dementia in the elderly. Currently, the application of traditional Chinese Medicine, such as huperzine A, ginkgo flavonoids, in the treatment of Alzheimer disorder has benefited many advantages over the conventional medicine obtained many achievements. Under the Chinese Medicine theory of the holism and syndrome differentiation, we developed a new recipe-the Natural Cerebrolysin (NC), a mixture drug extracted from Ginseng, rhizoma gastrodiae and gingko leaf at 2:1:1 ration for the treatment of mild cognitive impairment. In vitro experiments, the results showed that NC could protect B104 neurons against the neurotoxicities induced by beta-amyloid peptides. The expression of GRP78 were significantly increased while the Caspase-12 protein expression was significantly decreased after marrow mesenchymal stem cells treated with $\mathrm{NC}$. The $\mathrm{AD}$ model rats were set up by hippocampus injection of beta-amyloid peptide and the memory impairment of the $\mathrm{AD}$ model rats was validated by Morris water maze tests. Results showed that the learning and memory ability of rats in the NC group were significantly improved, neuronal apoptosis, deposition of beta amyloid and phosphorylated tau protein in hippocampus were decreased, meanwhile the expression of endoplasmic reticulum stress marker proteins PERK, CHOP were significantly reduced and the expression of GRP78/Bip increased, suggesting that NC's protective role in AD model rats involved in anti-apoptosis and anti-endoplasmic reticulum stress mechanism.
\end{abstract}




\title{
Testing a Structural Equation Model of Language-based Cognitive Fitness with Longitudinal Data
}

\author{
Elizabeth Moxley-Paquette ${ }^{1,2, \varpi, \dot{a}}$ and Gary Burkholder ${ }^{2}$ \\ ${ }^{1}$ Wasdell Academy Ontario, Canada \\ ${ }^{2}$ Walden University, USA
}

\begin{abstract}
Development of language is taken for granted by most. Problems with language development can result in stress for the individual and family; there is a challenge in that the contemporary education system assumes children have appropriate skills when they begin school. The purpose of the study is to further test a theoretical model of language readiness known as language-based cognitive fitness, which includes measures associated with structural concepts of language involving verbal reasoning ability, visual synthesis, and active analysis that explained $91 \%$ of the variance in achievement, with longitudinal data. The sample includes children from a private school who received an extensive battery of tests at admission and annually thereafter. Scores from 11 cognitive measures were used in a structural equation modeling framework to test the model derived from cross-sectional data with longitudinal data over three time points. Results from this longitudinal analysis demonstrated language-based cognitive fitness to be an interaction of verbal reasoning abilities, visual synthesis, and active analysis depending on the time period. There is a hint of hierarchical structure but it is a secondary consideration to the core model. Implications for positive social change include an improved understanding of the language structures responsible for language deficits and how these relate to overall cognitive fitness; and, the potential for interventions crafted to help children more quickly make up language deficits.
\end{abstract}

\section{Using the MMSE as a Cognitive Screener among Turkish and Moroccan Migrants}

\author{
Lennaert A.R. Zwart ${ }^{1, \varpi, `, ~ M i r j a m ~ G o u d s m i t ~}{ }^{1}$,Jos P. van Campen ${ }^{1}$, Caroline J. Rijkers ${ }^{1}$ and A. W. Wind $^{2}$ \\ ${ }^{1}$ Slotervaart Hospital, The Netherlands \\ ${ }^{2}$ Dutch Society of General Practitioners, The Netherlands
}

\begin{abstract}
The Dutch population of first generation Turkish and Moroccan migrants is ageing. Among them hypertension and diabetes mellitus are frequent findings, which will probably cause an increased incidence of dementia. The language barrier, low education and cultural differences make the diagnosis more difficult. To what extent the MMSE can be used as a cognitive screener in this population will be investigated in this retrospective study. Patients received standard diagnostics, a professional translator addressed the language barrier. Correspondence was scored on characteristics of dementia, a procedure was used to diagnose without including the MMSE score. The optimal cut-off was calculated with the Youden Index and Area under the ROC (AUROC).106 patients were included, 61\% had no education. Average MMSE-score was 16, with dementia 12, without 19. The AUROC was 0.85 , optimal cut-off was 18 with a Youden index of 0.61 . By using a professional translator the MMSE can possibly be used as a cognitive screener in this population. However, the cognitive skills addressed by the MMSE require an education and language skills. Developing a test that is independent of education and language barrier will probably be better.
\end{abstract}

\section{Prevalence of Domain-specific Cognitive Impairment in Patients with COPD}

\author{
Fiona Cleutjens ${ }^{1, \varpi, \AA,}$ Martijn Spruit ${ }^{1}$, Lowie Vanfleteren ${ }^{1}$, Frits Franssen ${ }^{1}$, Jeanette Dijkstra ${ }^{2}$, Rudolf Ponds ${ }^{2}$, Emiel \\ Wouters $^{1,3}$ and Daisy Janssen ${ }^{1}$ \\ ${ }^{1}$ Department of Research and Education, CIRO+, Centre of Expertise for Chronic Organ Failure, The Netherlands; \\ ${ }^{2}$ Department of Medical Psychology, Maastricht UMC+/School for Mental Health and Neurosciences (MHeNS), The Netherlands \\ ${ }^{3}$ Department of Respiratory Medicine, Maastricht UMC+, The Netherlands
}

\begin{abstract}
Background: Chronic obstructive pulmonary disease (COPD) has been associated with cognitive impairment. This may influence quality of life, physical activity level, treatment adherence, and hospital admissions. To date, the prevalence of cognitive impairment in COPD remains unknown. Further, it remains unknown which cognitive domains are affected. The aim of this study was to investigate the prevalence of domain-specific cognitive impairment in patients with COPD.
\end{abstract}


Methods: A detailed neuropsychological testing battery was administered in clinically stable COPD patients entering pulmonary rehabilitation. Six core tests used in the Maastricht Aging Study (MAAS), a large Dutch longitudinal study involving healthy individuals, were used to assess overall cognitive impairment ( $Z$ score below -1SD for at least two core tests). Compound $Z$ scores were constructed for psychomotor speed, planning, memory, and cognitive flexibility, and compared with the compound $\mathrm{Z}$ scores of MAAS, or normative data in scoring if compound scores of MAAS were not available. One-way ANOVA was used to compare cognition between patients with mild, moderate, severe or very severe airflow obstruction.

Results: 183 COPD patients (mean age 63.6 (9.4) years; 53\% men; mean FEV 54.8 (23.0) \% pred.) were included. 42\% showed overall cognitive impairment, and deficits in multiple domains were present: psychomotor speed (10\%), planning (11\%), memory (28\%), and cognitive flexibility (28\%). There were no statistically significant differences in cognitive functioning between patients with mild, moderate, severe or very severe airflow obstruction.

Conclusions: Irrespective of disease severity, cognitive impairment is prevalent in patients with COPD and presumably involves memory and cognitive flexibility.

\title{
Trends with Corticosteroid Use in Males with Duchenne Muscular Dystrophy Born 1982-2001
}

\author{
Deborah J. Fox ${ }^{1, \varpi, ఓ}$, Anil Kumar ${ }^{1}$, Nancy A. West ${ }^{2}$, A. Gregory DiRienzo ${ }^{3}$, Katherine A. James ${ }^{4}$, Joyce Oleszek ${ }^{5}$, and the \\ Muscular Dystrophy Surveillance, Tracking, and Research Network (MD STARnet) \\ ${ }^{1}$ New York State Department of Health, Albany, USA \\ ${ }^{2}$ Department of Epidemiology, University of Colorado School of Public Health, USA \\ ${ }^{3}$ Department of Epidemiology and Biostatistics, State University of New York at Albany, USA \\ ${ }^{4}$ Colorado Department of Public Health and Environment, USA \\ ${ }^{5}$ Department of Physical Medicine and Rehabilitation, University of Colorado School of Medicine, Children's Hospital Colorado, USA
}

\begin{abstract}
The benefit of corticosteroid use on motor power and muscular activities for boys with Duchenne muscular dystrophy (DMD) has been shown since the early 1980s. Following its introduction, however, treatment was generally advocated only until males became non-ambulatory as the focus was on prolonging ambulation. The 2010 Care Considerations, developed by panels of experts in the care of DMD, recommend starting corticosteroid treatment at the plateau phase, after developmental improvements have leveled off. Our study examines trends in corticosteroid use for males with DMD by birth year, race/ethnicity, and knowledge of a DMD family history. Firstborn males $(n=521)$ selected from a population-based surveillance system of DMD were analyzed using Kaplan Meier and regression methods. Comparing males born 1982 to 1986 with males born 1997 to 2001 , steroid use increased from $54 \%$ to $72 \%$ and mean age at steroid initiation decreased from 8.2 to 7.1 years. Hispanics and non-Hispanic Black males used steroids less frequently and delayed initiation compared to white males. Compared to males without a DMD family history, males with known family history were half as likely to use steroids - an unanticipated finding. Duration of steroid use increased over time and age at initiation decreased as expected. Racial/ethnic disparities exist for steroid use and should be addressed to improve outcome and quality of life for boys with DMD. Further study is warranted on reasons why males with a known family history were half as likely to use steroids.
\end{abstract}

\section{A Human Model: Changing the Focus on Autism Research}

\author{
Alysson Renato Muotri, ${ }^{1,2}$ \\ ${ }^{1}$ School of Medicine, Department of Pediatrics, University of California San Diego, USA \\ ${ }^{2}$ Department of Cellular \& Molecular Medicine, Stem Cell Program, Rady Children's Hospital San Diego, USA
}

\begin{abstract}
The unavailability of live human brain cells for research has blocked progress toward understanding mechanisms behind autism spectrum disorders (ASD). A human model, using reprogrammed patient somatic cells offers an attractive alternative as it captures a patient's genome in relevant cell types. Despite current limitations, the disease in-a-dish approach allows progressive time-course analyses of target cells, offering a unique opportunity to dissect cellular and molecular alterations before symptomatic onset. Understanding current pitfalls of this model is crucial for correct data interpretation and extrapolation of conclusions to the human brain. Innovative strategies to collect biological material and clinical information from large patient cohorts are important to increase the statistical power that allows extraction of information from the noise resulting from variability introduced by reprogramming and differentiation methods. Working with large patient cohorts is also important
\end{abstract}


to understand how brain cells derived from the diverse human genetic background respond to specific drugs, opening the possibility of a personalized medicine for ASD. I will present how induced pluripotent stem cells can be used to model ASD, revealing new insights into the fundamental mechanism behind autism.

\title{
Recognition of Homocysteine as a Risk Factor for Peripheral Neuropathy in Clinical Practice
}

\section{Jin Jun Luo}

Temple University School of Medicine, USA

\begin{abstract}
Introduction: Neuropathy may be caused by numerous etiologies, such as metabolic, infectious, inflammatory, toxic (including adverse effects of certain drugs and radiation), malnutritional, inherited, or autoimmune-mediated mechanisms. However, a large percentage (32-70\%) of all neuropathies remains "idiopathic" after a routine clinical investigation. Patients who were diagnosed with idiopathic neuropathy may have a heretofore unidentified etiology, which is less than optimally investigated. Recent clinical studies disclosed that an elevated plasma level of homosysteine (eHcy) exaggerates the prevalence of neuropathy in diabetics and exacerbates the preexisting diabetic neuropathy. The authors recently reported that eHcy is an independent risk factor for development of peripheral neuropathy and the electrophysiologic features of isolated eHcy-induced peripheral neuropathy (IHIN) are a mild large fiber sensorimotor neuropathy. However, IHIN is largely unrecognized clinically.
\end{abstract}

Objective: To demonstrate the spectrum of IHIN in adults.

Methods/Results: The authors' own clinical and neurophysiologic studies were reviewed to demonstrate that: (1) eHcy is an independent risk factor for development of IHIN; (2) clinical features of IHIN are of predominant sensory deficits; (3) the electrophysiologic features of IHIN are a mild large fiber sensorimotor neuropathy with mixed mild demyelination and distal axonal degeneration; and (4) central conduction slowing in IHIN disclosed by neurophysiologic study may be relevant to the pathophysiologic background for eHcy-induced central processing decline such as seen in dementia.

Summary/Conclusion: eHcy may cause peripheral and central nervous system dysfunction. Understanding the features of IHIN will help better manage the patients with eHcy and eHcy-related neurologic disorders.

\section{Pathobiologic Alterations in Preclinical Alzheimer's Disease}

\author{
Howard J Federoff ${ }^{1, \bullet,\llcorner, ~ M a s s i m o ~ F i a n d a c a ~}{ }^{1}$, Mark Mapstone ${ }^{2}$ and Amrita Cheema ${ }^{1}$ \\ ${ }^{1}$ Georgetown University Medical Center, USA \\ ${ }^{2}$ University of Rochester School of Medicine and Dentistry, USA
}

\begin{abstract}
Our group has studied a longitudinal cohort of seniors, $>70$ yo, to discover and validate peripheral blood measures that can accurate predict which cognitively normal subjects will progress to amnestic Mild Cognitive Impairment (aMCI) or Alzheimer disease $(\mathrm{AD})$ in a three year period. We initially reported on plasma metabolites which are now more accurate as a diagnostic. In addition, we have studied PBMC transcripts by RNAseq and plasma proteomics by aptamer capture. In this presentation, I will link these different data elements and describe the pathobiologic alterations that are observed in preclinical AD and discuss potential significance.
\end{abstract}

\section{Neuroinflammation and Chronic Neurodegeneration after Traumatic Brain Injury: New Therapeutic Target}

\author{
Alan I. Faden 0 \\ University of Maryland School of Medicine, USA
}

\begin{abstract}
It has long been claimed that prior traumatic brain injury (TBI) increases the subsequent incidence of Alzheimer disease (AD). However, recent larger epidemiological studies indicate a relationship to subsequent dementia but not to AD. There
\end{abstract}


is also a well-recognized association between repeated mild TBI and progressive cognitive decline or other neuropsychiatric abnormalities. The latter was first described in boxers as dementia pugilistica, and has received widespread attention in relationship to high contact sports. The term chronic traumatic encephalopathy (CTE) has been used to define a "specific" entity marked by neurobehavioral changes and deposition of phosphorylated tau protein. Less well appreciated with regard to post-traumatic neurodegeneration is the role of sustained neuroinflammation, even though this association has been recognized pathologically for decades. More recent experimental work, as well as clinical neuroimaging studies has underscored the relationship between chronic neuroinflammation and progressive brain neurodegeneration, while providing mechanistic support. Manifested by extensive microglial and astroglial activation, such chronic traumatic brain inflammation (CTBI), or perhaps better termed chronic traumatic inflammatory encephalopathy (CTIE), appears likely to be the most important cause of post-traumatic neurodegeneration and related cognitive decline in terms of prevalence. Perhaps even more critically, emerging preclinical studies indicate that persistent neuroinflammation and associated neurodegeneration may be treatable weeks to months after the initiating insult(s).

\title{
Oxidative Stress in Rett syndrome: Cause or Consequence?
}

\author{
Joussef Hayek ${ }^{\square}$ \\ Child Neuropsychiatry Unit, University General Hospital, Azienda Ospedaliera Universitaria Senese, Italy
}

\begin{abstract}
Rett syndrome (RTT), a rare neurodevelopmental disorder affecting almost exclusively females, mainly caused by a mutation in the $\mathrm{X}$-linked methyl-CpG binding protein 2 (MECP2) gene. High circulating levels of oxidative stress (OS) markers in patients suggest the involvement of OS in the RTT pathogenesis. In fact, OS hypothesis is able to explain several features of RTT: OS imbalance is related to $M e C P 2$ gene mutation type, as well as natural history and clinical heterogeneity of the disease. Our recent study on oxidative brain damage in Mecp2 mutant mouse models provide direct evidence of the link between Mecp2 deficiency, OS and RTT pathology. Notably, our findings demonstrate the rescue of the brain oxidative homeostasis in brainspecifically Mecp2-reactivated mice, indicating the potential reversibility of the disease. Interestingly, our recent observations indicated that lipid homeostasis is also perturbed in MECP2 patients with a high serum cholesterol and an oxidative-mediated loss of HDL receptor SRB1 (scavenger receptor class B, type 1) in RTT fibroblasts. The results of this study point out as the down-regulation of SRB1 could explain some of the clinical features of RTT such as hyperlipidemia, lung infections and respiratory bronchiolitis. Further efforts in the near future are needed in order to dissect the "black box" of the molecular events likely linking the MECP2 gene mutation to OS derangement and subsequent disease expression and to identify potential new targets and interventional strategies aimed at improving the quality of life of these patients, affected by a so far incurable disease.
\end{abstract}

\section{Examining the Implementation of a Social Engagement Intervention for Children with Autism in Public School Settings}

\author{
Jill Locske ${ }^{-}$ \\ University of Pennsylvania, USA
}

\begin{abstract}
Although inclusion of children with autism is increasing, inclusion is necessary but not sufficient to improve social functioning. Few evidence-based interventions have been successfully implemented in schools because schools often face many challenges when using these programs. There is growing evidence that these interventions rarely are adopted or successfully implemented in public schools, in part because of the lack of fit between the intervention and the needs and capacities of the school setting. Conducting research in partnership with schools increases the potential that the results will be more relevant to the setting, that schools will actually use the intervention, and that its use will result in positive outcomes for students. There is little systematic information available regarding the barriers to implementation of complex interventions such as those addressing social engagement for children with autism. The present study used field notes from an implementation trial to explore barriers that emerged during the training of school personnel and subsequent implementation of a social skills intervention. A number of barriers at the individual (training) and school levels (policies surrounding recess, staffing, prioritization of competing demands, level of respect and support, and availability of resources) interfered with the continued use and sustainment of the intervention. We offer potential strategies to overcome these barriers and provide directions for future research in this critical area.
\end{abstract}




\title{
Evidence-based Practices for Children with Autism Spectrum Disorders and Deafness
}

\author{
Stacey Jones Bock ${ }^{\square, \AA}$ and Christina Borders \\ Illinois State University, USA
}

\begin{abstract}
One in 59 deaf students has an additional diagnosis of Autism Spectrum Disorder (ASD). Either one of these disorders has great impact on language, behavior, and socialization. When there is comorbidity, there is a tremendous impact on the child. There have been virtually no research studies available on interventions for this population. The field of ASD has over 50 years of research on intervention practices that prove effective on students with ASD. The field of deafness does not have this supporting literature. The field of ASD is certainly the place to begin because we cannot ignore the impact of ASD on a student who is deaf. There are 5 evidence-based practices that can be used as a starting place to use with students who are deaf with ASD. Some practices will need to be modified based upon individual student characteristics. This presentation will overview these practices which include functional behavior assessment, functional communication training, reinforcement, visual and environmental supports, and the use of choice. We will also provide future direction for not only intervention but research as well.
\end{abstract}

\section{Teaching Advance Movement Exploration Skills in Water to Children with Autism Spectrum Disorders}

\author{
Mehmet Yanardag1,®, «, Mert Erkan², Ilker Yılmaz ${ }^{2}$, Ela Arıcan² and Ayten Duzkantar ${ }^{1}$ \\ ${ }^{1}$ Research Institute for Individuals with Disability, Anadolu University, Turkey \\ ${ }^{2}$ Faculty of Sport Sciences, Anadolu University, Turkey
}

\begin{abstract}
This study evaluated the effectiveness of the 'most to least' prompting (MLP) procedure on the teaching of advance movement exploration skills in water to children with autism spectrum disorders (ASD). Three 6-year old children with ASD, participated in the study and were taught 3 different aquatic skills, essential for movement exploration in water and swimming, in a one-to-one training format at three sessions per week. A multiple probe design across behaviors was used and was replicated across subjects to analyze the effects of MLP. The results of the study showed that MLP was effective in teaching advance movement exploration skills in water to children with ASD. Performing the exploration skills in water was continued after the training process during maintenance and generalization probe sessions. In addition, social validity results reflected that parents' opinions were positive on the learning skills in terms of functionality, beginning swimming and participation in other aquatic settings for their children. The enjoyable intervention and appealing setting are recommended to increase the repertoire of leisure skills and level of physical activity for children with ASD.
\end{abstract}

\section{Treatment of Autism with Low-dose Phenytoin}

\author{
Philip Bird" \\ Gosforth Clinic, Australia
}

\begin{abstract}
The drug treatment of autism spectrum disorders is often poorly tolerated and has limited benefit for the core social deficits. I will present the novel use of a low dose of the anti-epileptic, phenytoin to enhance social functioning in a 19-yearold Caucasian man with autism spectrum disorder treated with stimulant medication since early childhood. He experienced long-standing difficulties in establishing and maintaining relationships and reading social cues. Within 10 minutes of a single sublingual low dose of phenytoin there was an immediate observable improvement in his eye contact and enhanced integration of both verbal and non-verbal communication. These benefits were associated with his adherence to the low-dose phenytoin therapy which was maintained for over 18 months of follow-up. While acknowledging this is a single case, I have also observed similar improvements in other patients with social cognitive deficits treated with low dose phenytoin and other low dose anti-epileptic medications. I will describe my clinical experience and hypothesize that low-dose phenytoin may enhance GABA neurotransmission thereby correcting the GABA deficit which has been thought to be involved in autism and related developmental conditions.
\end{abstract}




\title{
The Role of Neuroinflammation in Tau Pathology; Therapeutic Perspective
}

\author{
Maj-Linda B. Selenica, ${ }^{1,2, \varpi, ¿, ~ H a y k ~ D a v t y a n ~}{ }^{3,4}$, Steven B. Housley ${ }^{2,5}$, Awa Sanneh ${ }^{1,2}$, Joseph Liu ${ }^{1,2}$, Laura J. Blair ${ }^{2,6}$, Anne \\ Gillies $^{6}$, Bryce A. Nordhues ${ }^{2,6}$, Bo Zhang ${ }^{2,6}$, Jason E Gestwicki ${ }^{6}$, Kevin Nash ${ }^{2,5}$, Marcia N. Gordon ${ }^{2,5}$, Dave Morgan ${ }^{2,5}$ and $^{2}$ \\ Chad A. Dickey ${ }^{2,6}$
}

${ }^{1}$ College of Pharmacy, University of South Florida, USA

${ }^{2}$ USF Health, Byrd Alzheimer Institute, USA

${ }^{3}$ Institute for Molecular Medicine, USA

${ }^{4}$ Institute for Memory Impairments and Neurological Disorders, UCI, USA

${ }^{5}$ Morsani College of Medicine, University of South Florida, USA

${ }^{6}$ University of Michigan, USA

\begin{abstract}
Accumulation of the microtubule associated protein tau in the brain is linked to a number of neurodegenerative diseases termed tauopathies. The most common of these is Alzheimer's disease (AD). Mice with transgenic insertion of tau mutations (i.e. P301L) have provided invaluable pre-clinical tools to study tau pathogenesis. Studies using these mouse models have shown that removing tau could be beneficial for disease symptoms. Developments in tau-targeted immunotherapeutic strategies have suggested that both active and passive immunization against tau can be advantageous. Using active immunization paradigm we have created an epitope map that revealed the emergence of a novel tau immunogen by peptide array approach. Our studies also suggest that active vaccination reduced levels of inflammation in CNS of transgenic mice. Evidence of brain inflammation has been repeatedly shown in $\mathrm{AD}$ animal models and postmortem brain tissue of $\mathrm{AD}$ patients. Currently, we have undertaken studies to understand the role of inflammation in a transgenic animal model of tauopathy. Stimulation of CNS with inflammatory molecules provokes microglia activation in CNS that is translated into increases in levels of phosphorylated and aggregated but not soluble tau forms. This study and others suggest that a synergistic effect between inflammation and tau pathology seem to take place in the diseased brain, albeit the exact mechanism is unknown. Targeting the mechanistic pathways involved in the transmission of pathology by glial cells could be an appealing approach.
\end{abstract}

\section{Plaque Array and Particles Targeted Proteomics Based Identification of Serum Biomarkers for Alzheimer's Disease}

\section{Shanmugavel Madasamy}

Plaxgen Inc., USA

\begin{abstract}
A hallmark of Alzheimer's disease (AD) is abnormal accumulation of amyloid plaques in regions of brain and cerebral arteries. $\mathrm{AD}$ remains mostly unresponsive to currently available therapeutics. Part of the challenging scientific task is due to lack of a predictive method to detect early stage of $\mathrm{AD}$ when therapeutic intervention may prove effective. Thus the development of a biomarker-based approach will significantly aid in the early diagnosis of AD. Here we describe a novel plaque array method based serum biomarker identification. The plaque array method employs flow cytometry in combination with mass spectrometry for efficient detection, sorting and proteomics analysis of serum derived plaque particles. As a result, 195 serum proteins that participate in the process of in vitro plaque particle formation have been identified. Among the 195 proteins, $68.2 \%$ of them overlapped in abeta-42, cholesterol, tau-275 and $\alpha$-synuclein plaque particles. Significantly, $22.5 \%$ of the proteins identified as bound to abeta-42 plaque particles generated in $\mathrm{AD}$ serum were unique when compared with cholesterol, $\alpha$-synuclein and tau plaque particles. In age-matched controls, $15 \%$ of them showed in vitro insoluble abeta- 42 particles formation and also $59 \%$ of the identified constituents from AD serum were present in the insoluble plaque particles of the control. In conclusion, by applying the plaque array method and targeted proteomics approach we have identified serum response proteins against an array of plaque forming aggregates related to AD pathogenesis. With further clinical validation, this assay may provide a novel, noninvasive means for the early detection and stratification of AD.
\end{abstract}




\title{
Epileptic Encephalopathy Mutations in KCNQ2 Disrupt Axonal Enrichment of KCNQ Potassium Channels and Excitability in Hippocampal Neurons
}

\author{
John P. Cavaretta ${ }^{1}$, Dhruv Joshi ${ }^{1}$, Weilun Pang ${ }^{1}$, Shuwei Wang ${ }^{1}$, Mary Hong ${ }^{1}$, Kwan Young Lee ${ }^{1}$ and Hee Jung Chung ${ }^{1,2 \boxminus, \varkappa}$ \\ ${ }^{1}$ Department of Molecular and Integrative Physiology, School of Molecular Cellular Biology, University of Illinois at Urbana- \\ Champaign, USA \\ ${ }^{2}$ Neuroscience Program, University of Illinois at Urbana-Champaign, USA
}

\begin{abstract}
KCNQ channels are voltage-gated potassium channels composed of KCNQ2 and KCNQ3 subunits. Highly concentrated at the axonal surface where action potentials initiate and propagate, they potently inhibit repetitive and burst firing of action potentials, which is the hallmark for neuronal hyperexcitability leading to seizures. Indeed, KCNQ2 and KCNQ3 mutations cause benign familial neonatal convulsion and myokymia. We have shown that some of these mutations impair axonal enrichment of KCNQ channels by disrupting KCNQ2 binding to calcium-binding protein calmodulin, and blocking their trafficking from the endoplasmic reticulum (ER) to the axonal surface. Recently, de novo mutations in KCNQ2 were discovered in patients with epileptic encephalopathy, severe symptomatic drug-resistant epilepsy with severe psychomotor retardation. Half of these mutations were found in the KCNQ2 C-terminal tail that interacts with calmodullin. Here, we show that epileptic encephalopathy mutations in KCNQ2 disrupt the axonal enrichment of KCNQ channels by reducing KCNQ2 binding to calmodulin and retaining them in the ER. Since the ER resident chaperones mediate the retention of misfolded proteins in the ER and this process requires calcium in the ER lumen, we are currently testing if depletion of calcium from the ER lumen could promote the trafficking of these trapped mutant KCNQ channels from the ER back to the axonal surface. These data and on-going experiments will provide an insight into the etiology of KCNQ2-associated epileptic encephalopathy, and may lay a firm foundation for targeting the ER chaperones as a possible therapy for epilepsy.
\end{abstract}

\section{$\beta$-Amyloidogenic Peptides Protect against Herpes Simplex Virus-1 Infections}

\author{
Karine Bourgade ${ }^{\square}$ \\ University of Sherbrooke, Quebec, Canada
}

\begin{abstract}
The causes of Alzheimer's disease (AD) are still under debate. However, experimental data and epidemiological studies suggest that Herpes Simplex Virus-1 (HSV-1) infection is one of the risk factors. HSV-1 stays latent in trigeminal neurons but can be reactivated under low immune status of the host. Extracellular senile plaques in brains of Alzheimer's patients contain the fibrillar form of $\beta$-amyloidogenic peptides $(A \beta)$ 1-40 and 1-42. Interestingly, herpetic encephalitis caused by Herpes viruses affects the same brain regions as $\mathrm{AD}$. However, the question remains whether $\mathrm{A} \beta$ fufill undiscovered physiological roles. In this connection, $A \beta$ display anti-bacterial and anti-yeast activities in vitro. We showed that A $\beta$ 1-40 and 1-42 also displayed antiviral activity against HSV-1 replication without being internalized. The action of A $\beta$ directly targeted HSV- 1 outside the cells by a mechanism that may involve interference with the integrity of HSV- 1 envelope. Therefore, A $\beta$ may belong to a novel class of antimicrobial peptides that protect the brain against enveloped viruses such as HSV-1. In this connection, the neuron cell line $\mathrm{H} 4$ produced time-dependent $\mathrm{A} \beta$ in response to HSV- 1 infection which was impaired by $\beta$-secretase inhibition. A $\beta$ also induced production of pro-inflammatory cytokines like IFNa, IL-1b and TNFa in U118-MG microglia cell line.
\end{abstract}

\section{Biomarkers and Disease Mechanisms in Neurological/Neurodegenerative Disorders}

\author{
Sadhana Sathaye ${ }^{\varpi, \Perp, ~ S a c h i n ~ P a t i l, ~ R u f i ~ T a m b e, ~ P a n k a j ~ J a i n, ~ P r i y a ~ G h u m a t k a r ~ a n d ~ V a i b h a v i ~ P e s h a t t i w a r ~}$ \\ Department of Pharm. Sci. and Tech., Institute of Chemical Technology, India
}

\begin{abstract}
Biomarker is an objectively measured characteristic of a normal as well as pathological biological process. Over the years, neurological and neurodegenerative diseases have been majorly described by clinical and neuropathological perspective. Identification and precise validation of the specific biomarkers would forestall or even prevent the progression of debilitating neurological disorders like Epilepsy, Parkinson's and Alzheimer's disease. Innovation and adoption of biomarkers would speed the development of new disease therapies through the use of "progression" markers to delineate the development and course
\end{abstract}


of a disease. Oxidative stress, mitochondrial dysfunction, neuroinflammation, apoptosis, altered electrical brain activity, etc. are pivotal players in the progression and intensification of neurological and neurodegenerative diseases like epilepsy and ADPD. Evaluation of archetype biomarkers like EEG, fMRI, SPECT, PET scans, GSK- $3 \alpha / \beta$, A $\beta$, tau, $\alpha$-synuclein, Tyrosine hydroxylase BDNF, GFAP, mitochondrial complex-I\&IV, mitochondrial PreP, TNF $\alpha$, caspase-3, etc. would prove to be very beneficial as early diagnostics and serve as newer therapeutic interventions. The role of nutrients, phytochemicals and new chemical entities in modulating these changes for arresting disease progression or alleviating the symptoms can be well applied for therapeutics of such disorders. It is imperative to identify valid biomarkers to develop patient tailored and individualized therapies offering a better quality of life to the patients. Optimal translation of preclinical findings to clinical studies necessitates robust and objective biomarkers capable of reducing the cost of discovery and validation of new therapies.

\title{
A Role for Inflammation in the Pathogenesis of Lyme Neuroborreliosis
}

\author{
Geeta Ramesh ${ }^{\varpi, ~}$ and Mario T. Philipp \\ Division of Bacteriology and Parasitology, Tulane National Primate Research Center, Covington, USA
}

\begin{abstract}
Lyme neuroborreliosis (LNB), caused by the spirochete Borrelia burgdorferi $(\mathrm{Bb})$, affects both the peripheral and the central nervous systems in 15\% of Lyme disease patients. In humans, LNB may present as meningitis and encephalomyelitis, cranial neuropathy, or acute radiculoneuritis. We hypothesized that $\mathrm{Bb}$ induces inflammatory mediators in glial and neuronal cells, and contributes to immune activation. Further, that this inflammatory context potentiates glial and neuronal damage and dysfunction. To test our hypothesis, we constructed an ex vivo model consisting of tissue explants of brain frontal cortex (FC) and dorsal root ganglia (DRG) of rhesus macaques, and allowed live Bb to penetrate these tissues. We identified intracellular cytokines and the phenotypes of producer cells in the above explants. Microarray analysis of total RNA from FC slices was performed. A causal role for inflammation in the pathogenesis of LNB was assessed by evaluating inflammatory changes in the brain, spinal cord and DRG, and spinal nerve conduction, in rhesus macaques that were inoculated intrathecally with live Bb and treated with one of two anti-inflammatory drugs, dexamethasone or meloxicam, or left untreated. We also set up cultures of rhesus DRG cells, and astrocytes and microglia from rhesus FC, as well as human oligodendrocytes, and incubated them with live $\mathrm{Bb}$. Culture supernatants were subjected to multiplex ELISA to detect immune mediators, while apoptosis was evaluated by the TUNEL assay. The above phenomena were also evaluated in the presence and absence of the anti-inflammatory drugs dexamethasone and meloxicam. The findings of these evaluations will be presented.
\end{abstract}

\section{ACAT1/SOAT1 as a Therapeutic Target for Alzheimer's Disease and Other Related Neurodegenerative Diseases}

\author{
T.Y. Chang ${ }^{\square, \imath}$ and Catherine C.Y. Chang \\ Department of Biochemistry, Geisel School of Medicine at Dartmouth, USA
}

\begin{abstract}
Acyl-coenzyme A:cholesterol acyltransferases/sterol O-acyltransferases 1 and 2 (ACATs/SOATs) are intracellular enzymes that convert free cholesterol to cholesterol esters for storage. Our laboratory identified the first ACAT gene (Acat1). ACAT1 is expressed in all tissues, while ACAT2 is mainly expressed in intestines and hepatocytes. ACATs are founding members of the membrane bound $\mathrm{O}$-acyltransferase enzyme family, which contain multiple membrane spanning domains, share similar catalytic sites, but are involved in a variety of biological processes. Our laboratory showed that in the 3xTG AD mouse (that expresses mutant human amyloid precursor protein hAPP, mutant presenilin-1, and mutant human Tau), Acat1 knockout reduced the contents of both hAPP and the Abeta peptide 1-42 (Abeta 1-42), and rescued the cognitive defects present in the AD mice. We next took an adeno-associated viral mediated infection approach to express anti-ACAT1 siRNA, and showed that Acat 1 knock down in the CNS of symptomatic 3xTG-AD mice caused significant reductions in hAPP and Abeta1-42 contents, to levels comparable to those found in the 3XTG-AD mice without Acat1. Most recently, we showed that Acat1 knockout or a small molecule ACAT1 inhibitor K604 stimulated autophagosome formation and increased lysosomal proteolysis in microglia and in neurons. A common event that occurs in several prevalent neurodegenerative diseases, including $\mathrm{AD}$ is the prominent presence of specific misfolded/aggregated proteins/peptides in certain region of the brain. The cellular clearance of misfolded/aggregated proteins/peptides involves autophagy-mediated lysosomal proteolysis. Overall, our results implicate that blocking ACAT1 may provide a novel way to benefit multiple neurodegenerative diseases including $\mathrm{AD}$.
\end{abstract}




\title{
Use of Paroxetine as a Neuroprotective Therapeutic
}

\author{
Joseph P. Steiner $匚$ \\ NIH/NINDS, USA
}

\begin{abstract}
There are severe neurological complications that arise from HIV infection, ranging from peripheral sensory neuropathy to cognitive decline and dementia for which no specific treatments are available. The HIV proteins secreted from infected macrophages, gp120 and Tat, are neurotoxic. We wished to screen for, identify and develop neuroprotective compounds relevant to HIV-associated neurocognitive disorders (HAND). We evaluated more than 2000 compounds that included FDA approved drugs for protective efficacy against oxidative stress-mediated neurodegeneration and identified many selective serotonin reuptake inhibitors (SSRIs) as potential neuroprotectants. Numerous SSRIs were then extensively evaluated as protectants against neurotoxicity as measured by changes in neuronal cell death, mitochondrial potential, and axodendritic degeneration elicited by HIV Tat and gp120 and other mitochondrial toxins. While many SSRIs demonstrated neuroprotective actions, paroxetine was potently neuroprotective ( $100 \mathrm{nM}$ potency) against these toxins in vitro and in vivo following systemic administration in a gp120 neurotoxicity model. Interestingly, the inhibition of serotonin reuptake by paroxetine was not required for neuroprotection, since depletion of the serotonin transporter had no effect on its neuroprotective properties. We determined that paroxetine interacts selectively and preferentially with brain mitochondrial proteins and blocks calcium-dependent swelling but had less effect on liver mitochondria. Additionally, paroxetine induced proliferation of neural progenitor cells both in vitro, as well as in vivo in gp120 transgenic animals. Therefore, SSRIs such as paroxetine may provide a novel adjunctive neuroprotective and neuroregenerative therapy to treat HIV-infected individuals and the associated HAND. In addition, paroxetine may also have broader application as a neuroprotective therapy to other neurological disorder as well.
\end{abstract}

\section{Oxysterols-multifaceted Players in Neurodegenerative Diseases}

\author{
Othman Ghribi \\ Department of Basic Sciences, University of North Dakota School of Medicine, USA
}

\begin{abstract}
Several line of findings point to a deleterious role of hypercholesterolemia and disturbances in cholesterol metabolism in neurodegenerative disease. Hypercholesterolemia is associated with increased formation of cholesterol oxidation products (oxysterols) as a mean to reduce excess levels of plasma cholesterol. Oxysterols can also increase by the action of oxidative stress that enhances oxidation of cholesterol to oxysterols. 24-hydroxycholesterol (24S-OHC) and 27-hydroxy cholesterol (27-OHC) are prominent members of the oxysterol family group. While $24-\mathrm{OHC}$ originates primarily from the brain, 27-OHC originates mostly from the circulation. We measured the concentrations of 27-OHC and 24-OHC in cholesterol-fed animal models for Alzheimer's disease and found that levels of both $24-\mathrm{OHC}$ and $27-\mathrm{OHC}$ markedly increase in the plasma. In the brain, the $24-\mathrm{OHC} / 27-\mathrm{OHC}$ ratio decreases. We also found that while $27-\mathrm{OHC}$ increases $\alpha$-synuclein and reduces tyrosine hydroxylase levels, 24-OHC reduces $\alpha$-synuclein and increases tyrosine hydroxylase levels in human neuroblastoma cells. Such data are of relevance to Parkinson's disease, a disease characterized by $\alpha$-synuclein accumulation and tyrosine hydroxylase levels reduction. As the concentrations of oxysterols are tightly regulated in the brain, a shift in the 24-OHC/27-OHC ratio may place the brain at risk of degeneration. As Alzheimer's disease and Parkinson's disease share overlapping features, we suggest that increased levels of 27-OHC and reduced levels of 24-OHC may be a common pathological marker for Alzheimer's and Parkinson's overlap.
\end{abstract}

\section{NADPH Oxidase Activation and 4-hydroxy-2-nonenal/aquaporin-4 Adducts as Possible New Players in Oxidative Neuronal Damage Presents in Drug-resistant Epilepsy}

\author{
Giuseppe Valacchi- \\ University of Ferrara, Italy
}




\begin{abstract}
A correlation between epilepsy and cellular redox imbalance has been suggested, although the mechanism by which oxidative stress (OS) can be implicated in this disorder is not clear. In the present study several oxidative stress markers and enzymes involved in OS have been determined. In particular, we examined the levels of 4-hydroxy-2-nonenal protein adducts (HNE-PA), a by-product of lipid peroxidation, and the activation of NADPH oxidase 2 (NOX2), as cellular source of superoxide (O2(-)), in surgically resected epileptic tissue from drug-resistant patients $(\mathrm{N}=50)$. In addition, we investigated whether oxidative-mediated protein damage can affect aquaporin-4 (AQP4), a water channel implicated in brain excitability and epilepsy. Results showed high levels of HNE-PA in epileptic hippocampus, in both neurons and glial cells and cytoplasmic positivity for p47(phox) and p67(phox) suggesting NOX2 activation. Interestingly, in epileptic tissue immunohistochemical localization of AQP4 was identified not only in perivascular astrocytic endfeet, but also in neurons. Nevertheless, negativity for AQP4 was observed in neurons in degeneration. Of note, HNE-mediated post-translational modifications of AQP4 were increased in epileptic tissues and double immunofluorescence clearly demonstrated co-localization of AQP4 and HNE-PA in epileptic hippocampal structures. The idea is that sudden, disorderly, and excessive neuronal discharges activates NOX2 with O2(-) production, leading to lipid peroxidation. The resulting generation of $\mathrm{HNE}$ targets $\mathrm{AQP} 4$, affecting water and ion balance. Therefore, we suggest that seizure induces oxidative damage as well as neuronal loss, thereby promoting neuronal hyperexcitability, also affecting water and ion balance by AQP4 modulation, and thus generating a vicious cycle.
\end{abstract}

Conclusions: With our findings there is clear evidence that genetic polymorphisms in UGT drug metabolizing enzymes contribute to variable pharmacokinetics having impact on the toxicity and plays a significant role in the steady state concentration of sodium valproate. This results can be applied in clinical practice for dosage individualization

\title{
Association of Prone Position with Sudden Unexpected Death in Epilepsy
}

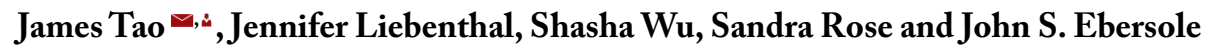

The University of Chicago, Chicago, USA

\begin{abstract}
Objective: Sudden unexpected death in epilepsy (SUDEP) usually occurs during sleep and in bed. Patients are commonly found in prone position. The objective of this study is to examine the association between prone position and SUDEP.

Methods: We conducted a systemic review and meta-analysis based on a literature search from databases Pub-med, Web of science and Scopus, using keywords "SUDEP" or "sudden unexpected death in epilepsy" or "sudden unexplained death syndromes in epilepsy." Twenty-five publications met the inclusion and exclusion criteria and were enrolled in this study.

Results: Body positions were documented in 252 SUDEP cases. Of these patients, $72.2 \%$ (95\% CI =66.3, 77.7) died in the prone position, whereas $27.8 \%(95 \% \mathrm{CI}=22.3,33.7)$ died in non-prone positions. Additionally, the prone position was reported in all 11 cases of video-EEG monitored SUDEP. Moreover, in a subgroup of 88 SUDEP cases where demographics and circumstances of death were documented, the prone position was observed in $85.7 \%$ (95\% $\mathrm{CI}=74.6,93.3)$ of patients under age 40 , but only in $60 \%(95 \% \mathrm{CI}=38.7,78.9)$ of patients above age 40 . Statistical analysis confirmed that the prone position was significantly more prevalent in the younger patient group, as compared to the older patient group (OR 3.9; 95\% CI=1.4, 11.4; $\mathrm{P}=0.009$ ).
\end{abstract}

Conclusion: There is a strong association between prone position and SUDEP, which suggested that prone position is a significant risk factor for SUDEP, particularly in patients under 40 years of age. As such, SUDEP may share mechanisms similar to sudden infant death syndrome (SIDS), therefore, is likely preventable.

\section{Prevalence of Drug Resistant Epilepsy in Adults with Epilepsy Attending a Neurology Clinic of a Tertiary Referral Hospital in Singapore}

\author{
Paul C. Ho ${ }^{1}{ }^{a, 4}$, Sing Teang Kong ${ }^{1}$, Choon Siang $\mathrm{Ho}^{1}$ and Shih-Hui Lim ${ }^{2,3,4}$ \\ ${ }^{1}$ National University of Singapore, Singapore \\ ${ }^{2}$ Singapore General Hospital, Singapore \\ ${ }^{3}$ National Neurosscience Institute, Singapore \\ ${ }^{4}$ Duke-National University of Singapore Graduate Medical School, Singapore
}




\begin{abstract}
The objective of study is to determine the proportion of population of adult people with epilepsy (PWE) in Singapore to have drug resistant epilepsy (DRE). 557 adult PWE who have attended the neurology specialist clinic of a tertiary referral hospital in Singapore were profiled for drug responses according to the definition for DRE as specified by the International League against Epilepsy (ILAE) 2010 consensus. This is a retrospective cohort study. Data collected included demographics, characteristics of seizure and epilepsy, blood biochemistry levels, electroencephalogram and brain imaging findings, and medication histories. The types and dosages of antiepileptic drugs (AEDs) used were retrieved from case notes and checked against pharmacy records. Each patient was counselled upon the diagnosis of epilepsy and taught to maintain a seizure diary. The dates and number of seizures were retrieved from these diaries at each visit. Treatment-related adverse effects were routinely assessed and hence, patients were assumed not to have treatment-related adverse effects when no relevant documentation was encountered. The prevalence rate of DRE in this clinic was $21.5 \%$. From multivariate analysis, patients with structuralmetabolic etiology, mental retardation, psychiatric illnesses and pre-treatment seizure frequency of more than once monthly were found to be more likely to have DRE $(p \leq 0.05)$. Although the influence of Indian ethnicity on the risk of DRE was only found in the univariate analysis, it warrants investigation in a larger cohort.
\end{abstract}

\title{
Choosing Anti-epilepsy Drugs by Seizure Characteristics: a Novel Concept
}

\author{
Paul H. McCabe \\ Geisinger Health Systemm, USA
}

\begin{abstract}
When the first 2 or 3 antiepilepsy drugs (AEDs) fail to stop a patient's seizures, we are left with the decision to try combination therapy or surgery. If surgery is not a viable option, then we are left with the former. Currently, the only advice of which two (or more) drugs to combine is to choose drugs with different mechanisms of action (i.e. "rational polytherapy"). Isobolography may suggest which AEDs may work better together. This data, however, is based on studies in animals. If data was available that could lead us to choose AED combinations based on the characteristics of a patient's epilepsy, it could lead to more effective and quicker seizure control. Both clinical features and study results are important in determining the type of epilepsy a patient has, but could they also play a role in determining the best treatment? Some possible observations may be a subset of temporal lobe epilepsy (TLE), frontal lobe epilepsy, and post-traumatic epilepsy. Due to cost and volume of patients needed to further investigate if certain combinations are more effective based on the characteristics of a patient's epilepsy, the best option would be collecting as much data as possible on as many patients as possible. Thus, having a national or international database to collect and analyze such data would be ideal. Such data could then also be used to evaluate other aspects of epilepsy, including genetic patterns.
\end{abstract}

\section{AMPA Receptor Antagonists for the Treatment of Epilepsy: a Novel Approach Based upon Auxiliary Proteins}

\author{
Jeffrey M. Witkin ${ }^{1,2, \square, 2, ~ A k i ~ K a t o ~}{ }^{1}$, Xingjie Ping ${ }^{2}$, JL Smith $^{2}$ and KM Gardinier ${ }^{1}$ \\ ${ }^{1}$ Lilly Research Labs, USA \\ ${ }^{2}$ Indiana University/Purdue University, USA
}

\begin{abstract}
AMPA receptors are a known target for the therapeutics of epilepsy for which blockade engenders antiepileptic efficacy. However, blockade of AMPA receptors globally in the brain results in characteristic side effects including dizziness and ataxia. Perampanel is the first AMPA receptor antagonist approved as an antiepileptic (2012). AMPA receptors are associated with auxiliary proteins including transmembrane AMPA receptor regulatory proteins (TARPS) that modulate the pharmacology and trafficking of AMPA receptors. We utilized these proteins to design an AMPA receptor antagonist that specifically targets AMPA receptors associated with TARP $\Delta-8$ but not TARP $\Delta-2$. TARP $\Delta-8$ has relatively high abundance in hippocampus and other forebrain areas, whereas, TARP $\Delta-2$ has highest regional localization in cerebellum. We hypothesized that an antagonist with selectivity of TARP $\Delta-8$ over TARP $\Delta-2$ would be an anticonvulsant (forebrain) without the motor side-effects (cerebellum) of non-TARP-dependent AMPA receptor antagonists which act across the entire brain. Whereas perampanel blocked AMPA-driven currents in rat and human hippocampus, cerebral cortex, and cerebellum, LY3130481, the first TARP $\Delta$-8-selective antagonist, only blocked effects in the hippocampus and cerebrum. In rodent models of acute and chronic (kindling) seizure provocation using chemicals or electrical stimuli, LY3130481, like perampanel was efficacious across a
\end{abstract}


broad range of assays but was devoid of motor side effects seen with perampanel. The efficacy of LY3130481 was shown to be dependent upon TARP $\Delta-8$ protein.

\title{
Alpha-synuclein Amyloid Interaction with Prion Protein: a Putative Overlap of Two Neurodegenerative Diseases
}

\author{
Giuseppe Legname \\ Scuola Internazionale Superiore di Studi Avanzati, Italy
}

\begin{abstract}
While the function of the cellular prion protein (PrPC) is still under debate, there are several reports indicating PrPC as being able to interact with $A \beta$ oligomers. Here, we investigate whether this is also true for $\alpha$-Synuclein ( $\alpha$-syn), protein involved in a group of pathologies collectively known as synucleinopathies. We formed recombinant mouse $\alpha$-syn amyloids using the same methodology employed to obtain synthetic mammalian prions. A combination of AFM (atomic force microscopy) and biochemical techniques was used to characterize the morphology and biochemical properties (such as resistance to proteinase $\mathrm{K}$ ) of $\alpha$-syn amyloids. Afterwards, we explored the uptake of $\alpha$-syn amyloids in neuroblastoma cell line: N2a cells which endogenously express PrPC (N2aWT), the overexpressing PrPC (N2aPrPFL), knocked out for PrPC (N2aKO), and scrapie infected N2a ( $\mathrm{ScN} 2 \mathrm{a})$ cells. Our results show that the uptake of $\alpha$-syn amyloids is lower in N2aKO if compared to control cells. Confocal microscopy and co localization with sub compartmental markers revealed that the $\alpha$-syn amyloids co-internalized with PrPC, accumulated and trafficked to lysosomes. Moreover, internalized $\alpha$ synamyloids were co-localizing with scrapie deposits in $\mathrm{ScN} 2$ acells. Further work was required to validate the importance of this interaction in disease progression invivo. Thus, we performed stereotaxic injections of $\alpha$-syn amyloids in substantia nigra pars compacta and striatum in FVB PrPWT and FVB PrPKO mice. Our findings suggest a role for PrPC in regulating of $\alpha$-syn uptake, thus, evidencing a link between the two neurodegeneration associated proteins. This study suggests an overlap between prion disease and Parkinson's disease.
\end{abstract}

\section{Expression of One Important Chaperone Protein, Heat Shock Protein 27, in Neurodegenerative Diseases}

\author{
Xuekai Zhang, ${ }^{1, \varpi, \varkappa}$, Jing Shi ${ }^{1}$, Jinzhou Tian ${ }^{1}$, Andrew C Robinson ${ }^{2}$, Yvonne S. Davidson ${ }^{2}$ and David M. Mann ${ }^{2}$ \\ ${ }^{1}$ The 3rd Department of Neurology, Dongzhimen Hospital, University of Chinese Medicine, China \\ ${ }^{2}$ Institute of Brain, Behaviour and Mental Health, Clinical and Cognitive Neuroscience Research Group, University of Manchester, UK
}

\section{Abstract}

Introduction: Many neurodegenerative diseases are characterized by accumulations of misfolded proteins that can colocalise with chaperone proteins (for example, heat shock protein 27 (HSP27)), which might act as modulators of protein aggregation.

Methods: The role of HSP27 in the pathogenesis of neurodegenerative disorders such as frontotemporal lobar degeneration (FTLD), Alzheimer's disease (AD) and motor neuron disease (MND) was investigated. We used immunohistochemical and Western blot analysis to determine the distribution and amount of this protein in the frontal and temporal cortices of 175 subjects, which composed of 72 patients with FTLD, 46 with AD, 25 with MND and 32 controls. FTLD fulfilled LundManchester criteria, AD fulfilled NINCDS-ADRDA criteria, and MND fulfilled El Escorial revised criteria for MND diagnosis.

Results: HSP27 immunostaining presented as accumulations of granules within neuronal and glial cell perikarya. Patients with AD and FTLD were affected more often, and showed greater immunostaining for HSP27, than patients with MND and controls. In FTLD, there was no association between HSP27 and histological type. The neuropathological changes of FTLD, $\mathrm{AD}$ and MND were not immunoreactive to HSP27. Western blot analysis revealed higher HSP27 expression in FTLD than in controls, but without qualitative differences in banding patterns.

Conclusions: The pattern of HSP27 immunostaining observed may reflect the extent of ongoing neurodegeneration in affected brain areas and is not specific to FTLD, AD or MND. It may represent an accumulation of misfolded, damaged or unwanted proteins, awaiting or undergoing degradation. 


\title{
Amphetamine and $\beta$-phenylethylamine Cause Locomotor Effects through Activation of an Amine-gated Chloride Channel
}

\author{
Lucia Carvelli" \\ University of North Dakota School of Medicine, USA
}

\begin{abstract}
Amphetamine is a psychostimulant which is thought to generate its effects by promoting release of dopamine through reverse activation of the dopamine transporters. However, some amphetamine-mediated behaviors persist in dopamine transporters knockout animals, suggesting the existence of alternative targets. We found that in C. elegans, a large fraction of the behavioral effects of amphetamine is mediated by activation of the newly identified amine-gated chloride channel LGC-55. Our in vitro and in vivo results demonstrate that amphetamine generates behavioral effects by both increasing the extracellular dopamine levels through the dopamine transporter and by activating the LGC-55 channels. Furthermore, our data demonstrate that the endogenous trace amine $\beta$-phenylethylamine ( $\beta$ PEA) activates the LGC-55 channels and promotes behavioral effects more efficiently than amphetamine. Interestingly, we found that amphetamine potentiates the activation of the LGC-55 by $\beta \mathrm{PEA}$. Since $\beta \mathrm{PEA}$ is an endogenous amine, our data support the hypothesis that amphetamine interfere with the endogenous activation of LGC-55 by $\beta$ PEA. Though no mammalian homologue of the LGC- 55 receptors has been identified to date, a preliminary screen through the human protein data base has revealed the existence of 4 orphan proteins sharing 30-45\% identity with the LGC-55 at the amino acid level. Whether these receptors are activated by amphetamine or $\beta$ PEA needs to be established by future studies. Notwithstanding, our findings uncover alternative pathways engaged by amphetamine, and urge rethinking of the molecular mechanisms underlying the effects of this highly addictive psychostimulant drug.
\end{abstract}

\section{Association of Serotonin Transporter Gene (5HTT) Polymorphism and Juvenile Myoclonic Epilepsy: A Case-control Study}

\author{
Eman H. Esmail ${ }^{1, \square, \varkappa, ~ D a l i a ~ M . ~ L a b i b ~}{ }^{1}$ and Walaa A. Rabie ${ }^{2}$ \\ ${ }^{1}$ Neurology Department, Faculty of Medicine, Cairo University, Egypt \\ ${ }^{2}$ Clinical and Chemical Pathology Department, Faculty of Medicine, Cairo University, Egypt
}

\begin{abstract}
Background: Serotonin levels might alter susceptibility to seizures. Serotonin transporter (5HTT) gene polymorphisms were found to be associated with some forms of epilepsy.

Objectives: We tried to examine an association between 5HTT VNTR allele variants in serotonin transporter gene and epileptogenesis in cases of Juvenile myoclonic epilepsy (JME). Methods: We conducted a case-control candidate gene study evaluating the frequencies of 5HTT VNTR allele variants using the SYBR Green real time PCR with melting curve analysis in JME patients and healthy subjects. 40 cases with JME were selected from the Epilepsy Outpatient Clinic of Kasr Al Ainy Hospital, Cairo University, classified according to the electroclinical classification of the ILAE. The control group consisted of 40 healthy Egyptian subjects.
\end{abstract}

Results: The less efficient transcriptional genotypes for 5-HTT polymorphisms were more frequent in JME patients (OR: 9.33, C.I.: 2.85- 30.60; p value < 0.001).

Conclusion: In our study we detected an association between the presence of 5-HTTVNTR less transcriptional efficient genotypes and JME, which suggests that modulation of the serotoninergic system might be implied in epileptogenesis in JME.

\section{Post Status Epilepticus Treatment with the Cannabinoid Agonist WIN 55,212-2 Prevents Chronic Epileptic Hippocampal Damage in Rats}

\author{
Roberto Di Maio $^{1,2, \square, \varkappa}$, Jason R. Cannon ${ }^{3}$ and J. Timothy Greenamyre ${ }^{1}$ \\ ${ }^{1}$ Pittsburgh Institute for Neurodegenerative Diseases and Department of Neurology, University of Pittsburgh, USA \\ ${ }^{2}$ RiMED Foundation, Palermo, Italy \\ ${ }^{3}$ School of Health Sciences, Purdue University, USA
}




\begin{abstract}
Repeated seizures are often associated with development of refractory chronic epilepsy, the most common form of which is temporal lobe epilepsy. $\mathrm{G}$-protein-coupled cannabinoid receptors $\left(\mathrm{CB}_{1}\right.$ and $\mathrm{CB}_{2}$ receptors) regulate neuronal excitability and have been shown to mediate acute anticonvulsant effects of cannabinoids in animal models. However, the potential of cannabinoids to prevent chronic neuronal damage and development of epilepsy remains unexplored. We hypothesized that treatment with a $\mathrm{CB}$ receptor agonist after an episode of status epilepticus - but before development of spontaneous recurrent seizures - might prevent the development of functional changes that lead to chronic epilepsy. Using the rat pilocarpine model, a therapeutic approach was simulated by administering the CB agonist, WIN55,212-2 after an episode of status epilepticus. Epileptic behavior was monitored during development of spontaneous recurrent seizures for up to 6 months. Histology, neurochemistry, redox status and NMDA receptor subunit expression were assessed at 6 months after pilocarpine-induced seizures. Sub-acute treatment with WIN 55,212-2 (for 15 days starting 24 hours after PILO injection) dramatically attenuated the severity, duration and frequency of spontaneous recurrent seizures. Further, in contrast to vehicle-treated animals, hippocampi from WIN55,2122-treated animals showed: normal thiol redox state, normal NR2A and NR2B subunit expression, preservation of GABAergic neurons and prevention of abnormal proliferation of GABAergic progenitors. This study shows for the first time that, after a known inciting event, treatment with a compound targeting $\mathrm{CB}$ receptors has the potential to prevent the epileptogenic events that result in chronic epileptic damage.
\end{abstract}

\title{
Complex Phenomenon of Suicide: Neurobiology and Clinical Aspects of the Unmet Public Health Dilemma
}

\author{
Mariyah Z. Hussain ${ }^{1, \square, \AA, ~ W a s h d a v e ~}{ }^{2}$, Sara Rasheed Dar ${ }^{3}$, Saeed Ahmed ${ }^{4}$, Suneeta Kumari ${ }^{5}$, Rizwan Ahmed ${ }^{6}$, Mustafa \\ Qureshi ${ }^{7}$, Satneet Singh $^{8}$ and Tazeen Azfar ${ }^{9}$
}

${ }^{1}$ Foundation University Medical College, Pakistan

${ }^{2}$ Sindh Medical College, Pakistan

${ }^{3}$ FMH College of Medicine and Dentistry, Pakistan

${ }^{4}$ Nassau University Medical Center, USA

${ }^{5}$ Howard University Hospital, USA

${ }^{6}$ Liaquat Medical E Dental College, Pakistan

${ }^{7}$ Brookdale University Hospital, USA

${ }^{8}$ Cooper University Hospital, USA

${ }^{9}$ Baqai Medical University, Pakistan

\begin{abstract}
Suicide is a complex phenomenon determined by the interaction among various factors such as neurobiology, stressful events, personal familial history, and other socio-cultural environment factors. However, the majority of suicides typically occur in the context of psychiatric disorders or due to the presence of severe medical challenges. Additionally, the global distress (the socioeconomic conditions due to the existing economic crisis) has also contributed to the increased rates of suicidal ideation and behaviors. Suicide remains a critical health challenge, where statistics show that about one million people across the world commit suicide every year. Literature and published statistics show that over $90 \%$ of those individuals who commit suicide suffer from mental disorders prior to their death. These high figures call for an alarming situation where the clinicians have to adhere to more responsibility and systematic approach which will enable them to recognize and prevent the catastrophic events leading to suicide. Therefore, the purpose of this article is to discuss available evidence regarding various aspects like prevalence of suicide, its theoretical advances, risk assessment, prevention strategies, neurobiological findings, and treatment strategies. We provide a critical assessment of the published literature in the effort to assist clinicians to effectively encounter suicidal patients in their daily practices.
\end{abstract}

\section{Anti-fibrillogenic and Fibril Destabilizing Effects of Metal Ions for Goat Brain Cystatin (GBC) Fibrils in vitro}

\author{
Waseem Feeroze Bhat ${ }^{\square,\llcorner}$ and Bilqees Bano \\ Department of Biochemistry, Faculty of Life Sciences, Aligarb Muslim University, India
}

\begin{abstract}
Transition metals such as zinc, iron and copper are emerging as major contributors to the complex chemistry and biology
\end{abstract}


of the brain in stages of health, aging and disease, because of the unique physiology, specialization, and complexity of brain tissue. The relationship between the development of neurodegenerative diseases and particular metal ions is very complicated and complex. Metal ions, especially copper, zinc and iron play very important roles in neurodegeneration having impact on both protein structure (misfolding) and oxidative stress. The present study focuses to highlight how metal ions $(\mathrm{Cu} 2+, \mathrm{Fe} 3+$ and $\mathrm{Zn2+}$ ) influence amyloid formation at low $\mathrm{pH}$ and on preformed amyloid fibrils. By employing thioflavin T assay, 4,4-Bis(1anilinonaphthalene 8-sulfonate), Congo red, $\mathrm{CD}$ and microscopy to elucidate the effect of $\mathrm{Cu} 2+, \mathrm{Fe} 3+$ and $\mathrm{Zn} 2+$ on $\mathrm{GBC}$ aggregation at low $\mathrm{pH}$. Results show that $\mathrm{Cu} 2+$ and $\mathrm{Fe} 3+$ inhibit fibril formation of $\mathrm{GBC}$ by promoting amorphous aggregates. While as $\mathrm{Zn} 2+$ exclusively promotes the fibril formation at low $\mathrm{pH}$ resulting in the formation of more ordered aggregates. Furthermore, the combined results of these complementary methods also suggested that $\mathrm{Cu} 2+$ and Fe $3+$ destabilize the $\beta$-sheet secondary structure of pre-formed amyloid fibrils of GBC.

\title{
Neuropharmacological Review of the Nootropic Herb Bacopa monnieri
}

\author{
Sebastian Aguiar ${ }^{\square, 2}$ and Thomas Borowski \\ Pitzer College Department of Neuroscience, Claremont Colleges, USA
}

\begin{abstract}
This review synthesizes behavioral research with neuromolecular mechanisms putatively involved with the low-toxicity cognitive enhancing action of Bacopa monnieri (BM), a medicinal Ayurvedic herb. BM is traditionally used for various ailments, but is best known as a neural tonic and memory enhancer (nootropic). Numerous animal and in vitro studies have been conducted indicating potential medicinal properties. Several randomized, double-blind, placebo-controlled trials have substantiated BM's nootropic utility in humans. There is also evidence for potential attenuation of dementia, Parkinson's disease, and epilepsy. Current evidence suggests BM acts via the following mechanisms-anti-oxidant neuroprotection (via redox and enzyme induction), acetylcholinesterase inhibition and/or choline acetyltransferase activation, amyloid- $\beta$ reduction, increased cerebral blood flow, and neurotransmitter modulation (acetylcholine [ACh], 5-hydroxytryptamine [5-HT], dopamine [DA]). BM appears to exhibit low toxicity in model organisms and humans; however, long-term studies of toxicity in humans have yet to be conducted. This review will integrate molecular neuroscience with behavioral research.
\end{abstract}

\section{Signaling in the Brain: Novel Targets for Neuroprotection?}

\author{
Francisco S. Cayabyab ${ }^{\square}$ \\ University of Saskatchewan, Canada
}

\begin{abstract}
For decades, it has been suggested that the excitatory nerve transmitter glutamate mediates most of the nerve excitotoxicity after stroke, but antagonists of glutamate receptors have been unsuccessful as neuroprotective agents in clinical trials. The neuromodulator adenosine is also known to be elevated after stroke, and recent studies from our lab suggest that prolonged stimulation of adenosine receptors may alter the composition of specific glutamate receptors. Specifically, we found that in our hypoxia/reperfusion injury model as well as in our in vivo stroke model, the adenosine A1 receptors induce changes in AMPA receptors (a class of glutamate receptors) that lead to AMPA receptors expressed on neuronal membranes with enhanced calcium permeability. We propose that this provides the cellular basis for the increased neurodegeneration in vulnerable brain regions, including the memory-forming center called the hippocampus. We also recently determined that hippocampal long term potentiation, a cellular substrate of learning and memory, shows significant attenuation in stroked animals compared to sham-operated control animals. We have also identified several intracellular signaling pathways downstream of adenosine A1 receptor signaling that could provide the basis for the development of novel stroke therapeutic drugs. Our on-going work may provide an explanation for the decades old mystery of delayed neuronal cell death that occurs days or weeks following the initial stroke injury.
\end{abstract}




\title{
The Value of Adding Somatosensory Stimulation to Mirror Therapy on Muscular, Sensorimotor and Daily Function in Stroke Patients: a Randomized, Placebo-controlled Study
}

\author{
Ching-yi Wu ${ }^{1, \bullet,\llcorner, ~ Y a-y u n g ~ L e e ~}{ }^{1}$, Keh-chung $\operatorname{Lin}^{2}$ and Yu-wei Hsieh ${ }^{1}$ \\ ${ }^{1}$ Chang Gung University, Taiwan \\ ${ }^{2}$ National Taiwan University, Taiwan
}

\begin{abstract}
Mirror therapy (MT) is an effective intervention approach to improve upper extremity motor functions for patients after stroke. However, the effects of MT on muscular properties and daily function are limited. To augment the treatment effects of MT, a hybrid intervention approach - MT combined with mesh glove (MG) afferent stimulation (MT+MG) - has been proposed. This single-blind, randomized, placebo-controlled study aimed to investigate the intervention effects of MT+MG on muscular properties, sensorimotor functions, and daily function in patients with stroke. Forty-eight individuals with chronic stroke participated in this study. The participants were randomly allocated to 3 groups: MT, MT+MG, and MT with sham MG stimulation (MT+Sham). The participants received training 1.5 hours/day, 5 days/week for 4 weeks. The outcome measures were muscular properties (muscle tone and stiffness), sensorimotor functions (Fugl-Meyer Assessment, revised Nottingham Sensory Assessment, and Box and Block test), and daily functional activity (Functional Independence Measure). The results showed that $\mathrm{MT}+\mathrm{MG}$ group demonstrated improved muscular properties, while the MT and MT+Sham groups did not. The participants in the MT+MG and MT+Sham groups, compared with those in the MT group, showed significantly greater improvement in the Box and Block test and the Functional Independence Measure. No other significant differences were found among the 3 groups. In summary, MT combined with MG may facilitate improvements in muscular properties, manual dexterity, and daily function.
\end{abstract}

\section{Stroke after Coronary Artery Bypass Surgery with and without Cardiopulmonary Bypass}

\author{
Javed Iqbal ${ }^{1, \square, \dot{a}}$ and Abdul Rehman Abid ${ }^{2}$ \\ ${ }^{1}$ Punjab Institute of Cardiology, Pakistan \\ ${ }^{2}$ Hamad Medical Corporation, Qatar
}

\begin{abstract}
Objectives: Stroke is a devastating complication of coronary artery bypass grafting (CABG). This study was carried out with the aim that the development of off pump coronary artery surgery decreases the incidence/adverse outcome due to extra corporeal circulation.

Methods: The data utilized in this study was extracted from a randomized controlled trial that was conducted from January 2006 to March 2007 at Punjab Institute of Cardiology. One hundred patients were included in 'on pump' group-A, and 100 patients in 'off pump' group-B.

Results: The mean age of the patients in group-A was $53.51 \pm 9.96$ years and in group-B it was $51.59 \pm 10.30$ years. Renal failure occurred in $21 \%$ patients of group-A as compared to $10 \%$ of group-B while Acute Myocardial Infarction occurred in significantly higher number of patients in group-B (11\%) as compared to group-A (2\%). Off pump was associated with significant decrease in rate of stroke $1.5 \%$ as compared to on pump 3.5\%. Similarly there was decreased in the risk of deaths after stroke $0 \%$ as compared to on pump $1.5 \%$ mortality after stroke. In group A $6(3.5 \%)$ patients developed stroke where as in group-B $2(1.5 \%)$ patients developed stroke out of these 6 patients there were 2 mortalities after stroke in group-A while in group-B there was no mortality after stroke within 30 days postoperatively.
\end{abstract}

Conclusion: Off pump CABG is associated with significantly decreased rate of stroke in comparison with the on-pump CABG.

\section{Impact of Bone Fracture on Stroke Recovery}

\author{
Hua Su叫 \\ University of California, USA
}




\begin{abstract}
Stroke is the leading cause of disability in adults and an important risk factor for bone fracture. Alternatively, stroke is one of the most devastating complications of bone fracture. In the United States, approximately 70,000 stroke victims suffer from bone fracture within the first year after their stroke. Reported date showed that about $0.2 \%$ to $4.1 \%$ patients suffer from stroke after hip surgery. However, the impact of bone fracture on stroke recovery has not been fully studies. We have studied the influence of bone fracture shortly before or after ischemic stroke on stroke recovery in a mouse model. We found that bone fracture increases alarmins and pro-inflammatory cytokines in the blood and increase stroke related injury as well as functional deficits through augmenting the neuroinflammatory response. Mice with stroke and bone fracture have more severe functional deficits, larger infarct sizes, and more CD68+ macrophages in the peri-infarct region than mice that have stroke only. Bone fracture also increases oxidative stress in the injury and promotes pro-inflammatory polarization (M1) of macrophage. Antiinflammatory therapies reduce the negative impact of bone fracture on stroke recovery. In a clinical study, we found hip fracture patients with advance age are prone to have ischemic stroke and higher CHADS2 score and neutrophil counts are risk factors for post-fracture ischemic stroke. Prior bone fracture history is an independent risk factor for ischemic stroke. These findings could help doctors identifying fracture patients that at risk of post-fracture stroke and providing preventive therapies to these patients before surgical fixation.
\end{abstract}

\title{
Cost-effectiveness of Dabigatran and Rivaroxaban Compared with Warfarin for Stroke Prevention in Patients with Atrial Fibrillation
}

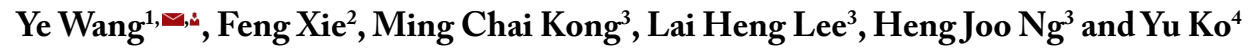 \\ ${ }^{1}$ Brigham and Women's Hospital, Harvard Medical School, USA \\ ${ }^{2}$ McMaster University, Canada \\ ${ }^{3}$ Singapore General Hospital, Singapore \\ ${ }^{4}$ Taipei Medical University, Taiwan
}

\begin{abstract}
Purpose: This study aimed to evaluate the cost-effectiveness of dabigatran and rivaroxaban compared with warfarin for the prevention of stroke in patients with atrial fibrillation (AF) in Singapore.

Methods: A Markov model was constructed to compare the lifetime costs, quality-adjusted life-years (QALYs) and incremental cost-effectiveness ratios (ICERs) of dabigatran 110 and $150 \mathrm{mg}$, rivaroxaban $20 \mathrm{mg}$ and adjusted-dose warfarin from the perspective of the Singapore healthcare system, using clinical data from published studies, utilities from a patientreported survey and costs from hospital databases. The target population was a hypothetical cohort of 65 -year-old AF patients with no contraindications to anticoagulation.

Results: In the base-case analysis, the QALYs were 8.75 with warfarin, 8.73 with dabigatran $110 \mathrm{mg}$, 8.82 with dabigatran $150 \mathrm{mg}$, and 9.33 with rivaroxaban. The costs were Singapore dollar (SG\$) 34,648 for warfarin, SG\$54,919 for dabigatran 110 $\mathrm{mg}, \mathrm{SG} \$ 50,484$ for dabigatran $150 \mathrm{mg}$ and SG $\$ 51,975$ for rivaroxaban. The ICER of rivaroxaban versus warfarin was $\mathrm{SG} \$ 29,697$ (US\$26,727) per QALY. Rivaroxaban and warfarin had extended dominance over the high-dose dabigatran. The low-dose dabigatran was dominated by warfarin. Deterministic sensitivity analyses showed that the ICER of rivaroxaban versus warfarin was sensitive to cost of rivaroxaban and utilities for rivaroxaban and warfarin. Probability sensitivity analysis demonstrated that the probability of rivaroxaban being the optimal choice was $97.8 \%$ and $99.5 \%$ at a willingness-to-pay threshold of SG\$65,000 (US\$58,500) and SG\$130,000 (US\$117,000) per QALY, respectively.
\end{abstract}

Conclusion: Rivaroxaban may be a cost-effective alternative to warfarin for the prevention of stroke in patients with AF in Singapore.

\section{CIMT:Theory of Research in Neurorehabilitation for Patients with Severe Brain Injury}

\author{
Ylenia Tripovic ${ }^{1, \varpi,\llcorner}$, Daniela Griguoli ${ }^{1}$, Francesco Formenti ${ }^{1}$ and Angela Marchese ${ }^{2}$ \\ ${ }^{1}$ Centro Adelphi, Italia \\ ${ }^{2}$ Osp. S. Spirito ASL RME, Italia
}

\begin{abstract}
The CIMT is a rehabilitative method devised by Prof. E. Taub applied on patients with brain stroke borrowing rigidly protocol CIMT, a multidisciplinary team of doctors, psychologists, occupational therapists and physiotherapists, has experienced
\end{abstract}


at the Day Centre Adelphi (Rome, Italy). The study is based on the observation of the results after application of induction therapy constrictive hemiplegic upper limb in a patient of 38 years, as a result of multiple injuries in road accidents (2003) (initial GCS = 3) has currently hemiplegia dx. The clinical case has met the criteria for inclusion in the protocol, applied for 15 consecutive days, 8 hours a day, in structured treatment settings and at home with the support of family. The motivation of the patient has resulted in encouraging results than the functional recovery of fine motor hemiplegic limb, specifically, the dominance in writing activities.

\title{
CIMT in Patients with an Outcome of Brain Injury after Stroke vs Post TBI
}

\author{
Francesco Formenti $^{1, \varpi,\llcorner}$, Ylenia Tripovic $^{1}$, Daniela Griguoli ${ }^{1}$ and Angela Marchese ${ }^{2}$ \\ ${ }^{1}$ Centro Adelphi, Italia \\ ${ }^{2}$ Osp. S. Spirito ASL RME, Italia
}

\begin{abstract}
This study proposes the application of the Protocol CIMT (Taub, 1993) to assess whether the outcome of patient with an outcome of brain injury after cerebral stroke, versus post TBI can be modified as a result of the application of the Protocol. The study was performed at the "Day Centre Adelphi" dedicated to integrated rehabilitation of GCA in Rome. Of a total of 30 patients referred regularly to the hospital, was selected a sample of 2 subjects ( 1 man, 1 woman, same age) whose characteristics have satisfied the inclusion criteria required (Mini Mental State, MAL); the sample was evaluated with five scales (WMFT, MAL, Barthel's Index, Abilhand scale) pre and post testing. The method involves the application of the CIMT Protocol for 15 days, (constriction of the healthy limb, and activation hemiplegic limb) through the execution of specific exercises, monitored by a multidisciplinary team (doctor, psychologist, TO and Physiotherapist), with the times so provided: 5 hours a day (therapy sessions at the centre), and 4 hours out of the centre (home skill assignment) for 15 days. Initial data argue in favor of a better average score for patients with an outcome of head injury (TBI) compared with post stroke. Further R.C.T. are desirable to confirm or refute this orientation.
\end{abstract}

\section{Detection of Atrial Fibrillation in Stroke Patients}

\section{Gerben J.J. Plas $₫$ ¿, Heleen M. den Hertog and Paul J.A.M. Brouwers}

Medisch Spectrum Twente, The Netherlands

\begin{abstract}
Atrial fibrillation (AF) is a strong risk factor for first ever stroke and stroke recurrence. Oral anticoagulation therapy with vitamin $\mathrm{K}$ antagonists or novel oral anticoagulants are very effective to prevent ischemic stroke in patients with $\mathrm{AF}$. Current stroke guidelines recommend at least 24 hours of electrocardiographic (ECG) monitoring besides a routine 12-channel ECG on admission. Since AF is often transient in nature, diagnosis may be missed with this approach. How AF should be diagnosed after stroke and by which methods and time frame is still a matter of debate. Recent studies suggests that silent AF is identified in approximately $25 \%$ of patients after cryptogenic stroke. Prolonged monitoring can improve these numbers. The additional detection rate of AF with prolonged ambulatory Holter monitoring for $1-7$ days is $10.7 \%$. Six months additional recording by mobile cardiac outpatient telemetry, external loop recording, or implantable loop recording shows an additional detection rate of $17 \% \mathrm{AF}$ in patients after stroke. This presentation will give an overview of detection techniques and rates of atrial fibrillation in patients after recent stroke. Moreover, the detection rate of an external loop recorder and factors to improve the diagnostic yield will be discussed.
\end{abstract}

\section{The Enigma of Relationship between Anemia and Pediatric Stroke; a Prospective Study Evaluating Relationship between Iron Status Parameters and Hematologic Indices with Pediatric Stroke}

\author{
Manish Parakh ${ }^{\varpi, \AA}$, Beerbal Khillery, Vanny Arora, Manisha Gurjar, S. Sridevi and Bindu Deopa \\ Dr. SN Medical College, India
}




\title{
Abstract
}

Anemia especially IDA is linked with all types of stroke but the correlation between various RBC indices and Iron status parameters with pediatric stroke has not been clearly established.

Objectives: To evaluate relationship between hematological indices and iron status with neurologic outcome, infarct size, number and location in children with Pediatric stroke. 55 children with acute stroke were enrolled. $74 \%$ patients had anemia, $66 \%$ had mild to moderate anemia and $8 \%$ had severe anemia. Low Serum ferritin $(<15 \mathrm{ng} / \mathrm{ml})$ was present in $22.6 \%$ patients. $17.1 \%$ patients with AIS had low serum Ferritin. Neurologic outcome at discharge was better in patients with normal platelet count at the time of stroke and poor in patients having severe anemia. During follow up from 3 month to 24 month, complete recovery was seen in $69.23 \%$ patients (vs none in severe anemia) and severe deficit in $7.69 \%$ patients not having anemia (vs $50 \%$ in severe anemia). In patients with normal serum ferritin complete recovery was seen in $58.33 \%$ (vs $42.85 \%$ in children with low serum ferritin) and severe deficit in $16.66 \%$ patients (vs no severe deficit but mild to moderate deficit in $57.14 \%$ patients with low serum ferritin). Area of diffusion restriction (ADR) on MRI brain was measured in 16 (8 male and 8 female) children with stroke as a surrogate marker of severity of infarction. No statistically significant correlation was seen between any of the RBC indices, total Platelet Count and serum Iron status indicators with ADr. in the whole cohort, in the group of patients having Serum Ferritin levels $<15$ and $>15 \mathrm{ng} / \mathrm{ml}$.

Conclusion: Anemia and low Ferritin levels are associated with poor neurologic outcome in Pediatric Stroke but there seems to be no correlation between the various RBC indices, total Platelet count and Iron status indicators with ADR.

\section{Atrial Fibrillation is Associated with Disturbed Glucose Metabolism in Patients with Ischemic Stroke and TIA}

\author{
E. Osei ${ }^{1, \sqsubset, \varkappa, ~ A . A . M . ~ Z a n d b e r g e n ~}{ }^{2}$, G.J.J. Plas ${ }^{1}$, P.J.A.M. Brouwers ${ }^{1}$, P.J. Koudstaal ${ }^{3}$, D.W.J. Dippel ${ }^{3}$ and H.M. den Hertog $^{1}$ \\ ${ }^{1}$ Medisch Spectrum Twente, Netherlands \\ ${ }^{2}$ Ikazia Ziekenhuizen, Netherlands \\ ${ }^{3}$ Erasmus Medisch Centrum, Netherlands
}

\section{Abstract}

Objectives: The prevalence of atrial fibrillation (AF) is increased in type 2 diabetes. Whether prediabetes and newlydiagnosed diabetes are risk factors forAF is controversial. We aimed to assess the prevalence of AF in patients with ischemic stroke or TIA with prediabetes, newly-diagnosed diabetes and pre-existent diabetes compared with normal glucose metabolism.

Methods: We included 1380 patients with TIA or ischemic stroke. In all nondiabetic patients, 2-hour post-load glucose levels were determined on day 2-5. We defined prediabetes and newly-diagnosed diabetes according to current guidelines. Preexistent diabetes was defined as the use of oral or parenteral anti-diabetic medication prior to admission. AF was defined as a combination of known AF prior to admission and registered AF during admission on an electrocardiogram or on 24-hour cardiac rhythm observation.

We studied the occurrence of AF in groups with different glucose metabolism. Multiple logistic regression was applied to study the associations between the glucose groups and AF.

Results: 114 patients (8\%) were known to have AF, and 38 (3\%) had de novo AF. 369 patients (27\%) had prediabetes, 274 (20\%) newly-diagnosed diabetes, and 293 (22\%) pre-existent diabetes. AF was more often present in patients with prediabetes $(10 \%, \mathrm{p}=0.010)$, newly-diagnosed diabetes $(15 \%, \mathrm{p}<0.001)$, and pre-existent diabetes $(12 \%, \mathrm{p}=0.001)$ than in those with normal glucose metabolism (5\%). Prediabetes (aOR 1.7; 95\% CI 0.8-3.6), newly-diagnosed diabetes (aOR 2.4; 95\% CI 1.2-5.0), and pre-existent diabetes (aOR 1.9; 95\%CI 0.9-4.0) were associated with AF.

Conclusion: Prediabetes and newly-diagnosed diabetes are risk factors for AF.

\section{VIT D3 as a Prognostic Factor in Cerebro Vascular Stroke}

\author{
N.S. Sampath Kumar ${ }^{\square, \varkappa}$, B. Venu Gopal and T. Sunanda \\ Narayana Medical College and Hospital, India
}

\begin{abstract}
Introduction: Stroke is the second most common cause of death and major cause of disability worldwide. Serum vit D3 is
\end{abstract}


one of the modifiable risk factor for stroke. There are few studies regarding role of vit D3 as a prognostic factor in Stroke.

Objectives: In this study, we investigated the risk factors for the stroke and role of vit D3 as a prognostic factor in Stroke.

Material and Methods: A total of 160 patients, who had cerebro vascular stroke, diagnosis was confirmed by CT and MRI, were evaluated prognosis by NIHSS score at the time of admission as well as discharge, $3 \& 6$ months after discharge and serum vit D3 was measured in all patients, plotted the prognosis against the controls.

Results: Out of total 160 patients of stroke, 129 had intracerebral infarct \& 31 had intracerebral hemorrhage. In stroke patients 40 had normal serum vit D3 levels ( $\geq 25$ micro grams per litre), 120 had low serum vit D3 levels (<25micro grams per litre). However there was significant low level of serum vit D3 in intracerebral infarct than intracerebral hemorrhage( $p$ value $=0.0496$ ). There were no significant relationship of low level serum vit $\mathrm{D} 3$ with prognosis of stroke.

Conclusion: The present study concluded that low level serum vit D3 seems to be a significant risk factor for stroke incidence particularly in intra cerebral infarct than hemorrhage. In addition to conventional risk factors, there were no significant relationship of low level serum vit D3 with prognosis stroke.

\title{
Public Knowledge of Cardiovascular Disease and its Risk Factors in Kuwait: A Cross- Sectional Survey
}

\author{
Abdelmoneim Awad ${ }^{\square, 2}$ and Hala Al-Nafisi \\ Department of Pharmacy Practice, Faculty of Pharmacy, Kuwait University, Kurwait
}

\begin{abstract}
Background: To design effective primary and secondary prevention programs, an assessment of a population's prior CVD knowledge is of paramount importance. This study was performed to assess the level of knowledge towards CVD types, warning symptoms of heart attack or stroke, and CVD risk factors.
\end{abstract}

Methods: A descriptive cross-sectional survey was performed using a pretested self-administered questionnaire on a sample of 900 randomly selected Kuwaiti individuals.

Results: The response rate was $90.7 \%$. Respondents' knowledge about types of CVD, heart attack or stroke symptoms was low. Almost $60 \%$ of respondents did not know any type of CVD, and coronary heart disease was the commonest identified type (29.0\%). Two-fifths of participants were not aware of any heart attack symptoms, and the most commonly known were chest pain (50.4\%) and shortness of breath (48.0\%). Approximately half of respondents did not recognize any stroke symptoms, and the most commonly recognized were 'confusion or trouble speaking' (36.4\%) and 'numbness or weakness' (34.7\%). Respondents' knowledge regarding CVD risk factors was moderate. The commonest factors identified by over four-fifths of participants were smoking, obesity, unhealthy diet and physical inactivity. In the multivariate logistic regression analysis, independent predictors of better level of CVD knowledge were females, age 50-59 years, high level of education, regular eating of healthy diet, and had a family history of CVD.

Conclusions: There are deficiencies in CVD knowledge among Kuwaiti population, which could turn into insufficient preventative behaviors and suboptimal patient outcomes. There is an apparent need to establish more wide-spread and effective educational interventions.

\section{Stimulation of NMDA Receptors in the Recovery Phase after Transient Ischemia in Rats: Outcomes and Mechanisms}

\author{
Anat Biegon ${ }^{\square, 2}$ and Jasbeer Dhawan \\ Stony Brook University, USA
}

\begin{abstract}
Ischemic stroke triggers a massive but transient glutamate efflux and activation of NMDA receptors, followed by long lasting loss of NMDA receptor function. We have recently reported that D-cycloserine (DCS), a partial NMDA agonist improves neurological and cognitive outcome in transient focal ischemia; although the mechanisms mediating these effects are not entirely clear. The present studies were designed to examine the effects of DCS on neuroinflammation, NMDA receptor density, BDNF and aromatase levels following transient focal ischemia in rats.
\end{abstract}


Rats subjected to a transient $(90 \mathrm{~min})$ Middle Cerebral Artery Occlusion (MCAO) were administered DCS (10 mg/kg, $\mathrm{N}=14$ ) or vehicle (PBS, N=14) 24 hours post reperfusion. Eight rats served as controls. Animals were killed more than 4 weeks after MCAO. Consecutive coronal cryosections were processed for quantitative autoradiography with the neuroinflammation marker [3H]PK11195 and the NMDAR antagonist [3H]MK801; and quantitative immunohistochemistry was used to assess $\mathrm{BDNF}$ and aromatase expression in the same brains.

DCS treatment reversed the decrease in NMDA receptor density and increased hippocampal BDNF, with no effect on neuroinflammation. Aromatase expression in cortical and striatal regions was increased by MCAO and reduced by DCS.

In conclusion, Single administration of DCS given $24 \mathrm{hrs}$ after ischemia normalized NMDAR density and increased BDNF in hippocampus of ischemic rats, suggesting that BDNF contributes to beneficial effects of DCS in stroke acting via a neuro-restorative rather than a neuroprotective mechanism.

\title{
Save YourPatients. Save YourPractice. FillYourPockets. The Power of Pharmacogenetics Testing
}

\author{
Cicely Everson $\square$ \\ American Academy of Anti-Aging Medicine, USA
}

\begin{abstract}
In the U.S. there are over 2 million adverse drug reactions (ADR) hospitalizations annually, with 106,000 of those resulting in death. Adverse drug reactions are considered the fourth leading cause of death in the United States. ADR's are reported to cost as much as $\$ 136$ billion dollars annually. The more drugs a patient takes, the higher the risk is of an ADR. Patients taking over 11 drugs have a $96 \%$ chance of an $\mathrm{ADr}$ and the average Medicare patient is on 5-8 drugs. In this seminar, the importance of pharmacogenetics testing and improved patient outcome is explored. The physician who offers pharmacogenetics testing is the physician who prescribes safer and more effective medicine, reduces the risk of side effects and overdoses, minimizes drug interactions, and knows very clearly how to find the right dose. Pharmacogenetics is the study of genetic differences or variations in metabolic pathways which can affect individual responses to drugs. This test predicts a patient's likelihood to experience an adverse event or not respond to a given drug. It can be used to greatly increase the safety and effectiveness of drug therapy. The patient becomes more confident in his physician's ability to find the right medication for his condition vs. the current trial and error approach in effect today. It is no surprise that several drugs can cause severe or life-threatening reactions in patients with certain genetic variations, and pharmacogenetics testing creates a personalized approach - for a safer patient and a safer practice.
\end{abstract}

\section{Pathological Patterns in Relapsing-remitting and Chronic Models of Multiple Sclerosis and the Therapeutic Effect of Glatiramer Acetate}

\author{
Rina Aharoni \\ The Weizmann Institute of Science, Israel
}

\begin{abstract}
Multiple sclerosis (MS) is a complex disease involving autoimmune inflammation as well as axonal and neuronal pathology. Yet, the roles of inflammatory versus degenerative processes in MS pathology are still controversial. Novel treatment strategies aim to act within the central nervous system (CNS) to reduce the inflammation and induce neuroprotection and repair processes. We analyzed the in situ pathological manifestations in two animal models of MS, the relapsing-remitting and the chronic experimental autoimmune encephalomyelitis (EAE), using immunohistochemistry, electron microscopy and advanced magnetic resonance imaging (MRI). The effect of treatment by glatiramer acetate (GA, Copaxone) on CNS damage and repair was studied in both models. Extensive analysis revealed multiple damages with different occurrence in the two EAE models. Hence, demyelination was characteristic to relapsing-remitting EAE, whereas in the chronic model axonal degeneration and neuronal loss were mainly manifested. In GA-treated mice, less demyelination as well as reduced axonal and neuronal injuries were found, even when treatment was applied in a therapeutic schedule, after the disease exacerbation, suggesting repair processes. Indeed, quantitative analysis revealed significant elevation in remyelination in GA-treated compared to untreated mice. Furthermore, neurogenesis, namely the birth of new progenitor neuronal stem cells was induced following GA-treatment. These cumulating findings support the notion that neuroprotective repair process in the CNS can be up-regulated by therapy.
\end{abstract}




\title{
Acute Pancreatitis: Possible Association of Dimethyl Fumarate for the Treatment of Relapsing-remitting Multiple Sclerosis
}

\author{
Hongxiu Luo ${ }^{\varpi, \AA}$, Harsh Bhatt, Shaza Mohamad, Eric Uhrik, Shuvendu Sen, Teena Mathew and Abdalla Yousif \\ Department of Internal Medicine, Raritan Bay Medical Center, USA
}

\begin{abstract}
Multiple sclerosis (MS) is a chronic inflammatory immune-mediated disease of the central nervous system with autoimmune demyelination and axon injury. Approximately $85 \%$ of the patients experience the relapsing and remitting MS (RRMS). Dimethyl fumarate (DMF, Tecfidera) was approved by the FDA in March 2013 for treatment of RRMS. The safety and tolerability of DMF was investigated in two phase III clinical trials, and its common adverse events include gastrointestinal, elevated hepatic aminotransferases, flushing and decreased white blood cell and lymphocyte count. However, Tecfidera-induced acute pancreatitis (AP) has never been reported. Herein, we describe a case of AP which was suspected to be induced by this new medication. The patient was a 43-year-old Hispanic female with 2-year history of RRMS. She developed acute pancreatitis 4 weeks after the treatment with Tecfidera $240 \mathrm{mg}$ PO twice a day. She had Cholecystectomy 5 year ago. The magnetic resonance cholangiopancreatography (MRCP) turned out to be negative for microlithiasis or biliary sludge with normal common bile duct and pancreatic duct caliber. The results of laboratory test included negative alcohol, normal serum levels of calcium and triglyceride. Patient's symptoms improved and lipase level turned to normal 3 days after we discontinued Tecfidera. In conclusion, Tecfidera-induced AP is easily masked and misdiagnosed, because gastrointestinal-related symptoms are the common side effects of Tecfidera. More clinical studies should be involved regarding the safety and tolerability of DMF.
\end{abstract}

\section{Cinnarizine for the Prophylaxis of Migraine Associated Vertigo: A Retrospective Study}

\author{
Foad Taghdiri ${ }^{1, \varpi, ¿, ~ M a n s o u r e h ~ T o g h a ~}{ }^{2}$, Soodeh Razeghi Jahromi ${ }^{3}$ and Farshid Refaeian ${ }^{4}$ \\ ${ }^{1}$ Iranian Center of Neurological Research, Neuroscience Institute, Tehran University of Medical Sciences, Iran \\ ${ }^{2}$ Department of Neurology, Neurology Ward, Sina Hospital, Tehran University of Medical Sciences, Iran Iranian Center of Neurological \\ Research, Neuroscience Institute, Tehran University of Medical Sciences, Tebran, Iran \\ ${ }^{3}$ Multiple Sclerosis Research Center, Neuroscience Institute, Tehran University of Medical Sciences, Tehran, Iran \\ ${ }^{4}$ Iranian Center of Neurological Research, Neuroscience Institute, Tehran University of Medical Sciences, Tehran, Iran
}

\section{Abstract}

Objective: to assess the efficacy and safety of cinnarizine for the prophylaxis of migraine associated vertigo in the vestibular migraine and migraine with brainstem aura.

Background: Vestibular migraine and migraine with brainstem aura are two principal clinical syndromes that frequently are associated with migraine. Since cinnarizine is a well-tolerated calcium channel blocker which has acceptable effect on both vertigo and migraine headache, we carried out this study to evaluate the efficacy and safety of this medication in vestibular migraine and also migraine with brainstem aura associated with vertigo.

Methods: This was a retrospective, single-center, open-label, investigation of the effects of cinnarizine on vestibular migraine and migraine with associated with vertigo. We assessed the change in monthly frequency of vertigo and also frequency, duration and intensity of migraine attacks after one, two and three months of cinnarizine administration.

Results: The mean frequency of vertigo and also the mean frequency, duration and intensity of migraine headaches per month were reduced significantly after three months of cinnarizine therapy (all $\mathrm{p}<0.001)$.

Conclusion: This study suggests that cinnarizine is safe and effective in reducing both headache and vertigo aspects of "migraine plus vertigo" among the patients who suffer from either vestibular migraine or migraine with brainstem aura associated with vertigo.

\section{Analytic Information Processing Style and Neurophysiological Correlates: Focus on Migraine and Cluster Headache}

\author{
Marzia Buonfiglio ${ }^{\square, \AA}$ and Francesco Di Sabato \\ Sapienza University of Rome, Italy
}

Journal of Neurology \& Experimental Neuroscience | Volume 1 Supplement 1, 2015 


\begin{abstract}
Analytic information processing style is characterized by the habit of continually evaluating and processing stimuli dealing with details and fine points. It has been linked to a continuous arousal state and higher brain activation (increased generalized gamma activity). A strong correlation between this specific information processing and both migraine and cluster headache $(\mathrm{CH})$, has been shown by our previous works, suggesting a potential role of analytic style in their pathogenesis. Moreover, we recently aimed to investigate a possible correlation between analytic style and lack of habituation, given that the latter is a trait marker of the "migraineur brain". We have demonstrated, for the first time, a significant relationship between lack of neural habituation and analytical information processing style, suggesting that it may be related to a disruption of brain energy balance. Given that habituation normalizes during migraine attack, the latter has been considered a defense mechanism against sensory overload, possibly linked to analytic cognitive style. Furthermore, we aimed to investigate cognitive behavior style of $30 \mathrm{CH}$ patients matched with a group of migraineurs without aura (MO)and healthy controls, evaluating analytic/global and visual/auditory dimensions of information processing style by Sternberg and Amos tests. Our results confirmed previous evidence and highlighted a predominant auditory analytic style in $\mathrm{CH}$, significantly different from the $\mathrm{MO}$ group, which scored higher in visual analytic style (ANOVA, $\mathrm{p}<0,0001$ ). This is a novel finding that warrants more in-depth research from a neurophysiological, neurobiological, and epigenetic perspective.
\end{abstract}

\title{
Changing Definitions of Euphoria in Multiple Sclerosis: A Short Report
}

\author{
Amy Duncan®^, Susan Malcolm-Smith, Ozayr Ameen and Mark Solms \\ University of Cape Town, South Africa
}

\begin{abstract}
A recent interest in euphoria in multiple sclerosis (MS) has resulted in a wealth of literature on this topic. However, a marked change in the definition of this symptom appears to have taken place since its first descriptions in the mid-19th century. This short report will demonstrate that the 'euphoria' being studied today may not be the same state as that originally observed and described in MS patients and some implications of this possibility are discussed.
\end{abstract}

\section{Cytokines in MS-Possible Targets for Immune Therapies}

\author{
Anastasiya Trenova" \\ Medical University - Plovdiv, Bulgaria
}

\begin{abstract}
The complex interactions between the central nervous system (CNS) and the immune system are mainly mediated via synthesis and secretion of cytokines. Violation of the fine balance between different cytokine subpopulations is undeniable factor in the development of pathological process in multiple sclerosis (MS). Experimental and clinical studies examining the impact of individual cytokine on the course of MS often give conflicting results, which demonstrates the compound connections of cytokines with each other and with the CNS. Yet there is little data on the role of cytokines in the advanced stages of the disease when neurodegeneration came to the fore. Efforts to modulate autoimmune process in MS have shown that effective treatments alter cytokine expression in the central nervous system (CNS) and in activated mononuclear cells, indicating that they are important therapeutic targets. The widely approved immune treatment with interferon-beta, glatiramer acetate, cytostatic drugs, but also experimental therapeutic agents as estradiol, statins and vitamin D have been proved to exert their beneficial effect partially via influence on cytokine production. Increasing knowledge of the role of cytokines in MS will create opportunities for a more precise determination of targets for immunologically active drugs in order to improve their effectiveness.
\end{abstract}

\section{BMT Monitors Neuropsychiatric Disorders with Bio-impedance Units}

\author{
Yakov Naisberg- \\ AMCHA-Netanya Branch, National Israeli Center for Psychosocial Support of Survivors of the Holocaust and the Second Generation, \\ Israel
}




\begin{abstract}
Background: The brain is an electro-energy generating source. My neuropsychiatric expertize predicted that: a) Microscopic defects on linear, parallel or/and cross regional ion channels of neuronal membranes across neuronal electric pathways (NEPs), may display different bio-impedance patterns in patients with positive and negative signs in schizophrenia. b) Spontaneous ionic discharges across a regional solitary or multiplex accumulated electrostatic field sources reaching sub seizure threshold with a low bio-impedance level in epilepsy. c) Chronic stress-distress strength cause singular or plural neuronal loop pathogenesis. It over-stimulate neuronal ionic information-processing fibers (wiring) to generate extra quantity of metabolic energy, heating up and extending brain tissue, pressurizing it against scull surfaces, with further cerebral edema (on ocular fundus) and cortical spreading depression (on EEG) in migraine. Hence, biophysical mind technology (BMT) is most likely to hold high potentials in treating and identifying $\mathrm{a}, \mathrm{b}$ and $\mathrm{c}$ pathology.
\end{abstract}

Methods: A small team with an electronic and computer software engineer built the BMT with multiplex EEG-like electrodes. Identical to EEG it operated on 10 to 20 montage of electrodes with harmless noninvasive induced through the scalp currents. BMT operated due to International medical devices regulation and local and central Helsinki committee. The first investigated cluster consisted of chronic hospitalized subjects, [16 with positive signs and 17 with negative signs of schizophrenia], 18 with epilepsy and 16 healthy controls. These patients were routinely treated with psychiatric and neurological drugs and measured with bio-impedance units under a double blind method. Many years later the second investigated cluster of severe migraine patients (30 experimental and 30 SHAM controls) underwent BMT with a treatment module and diagnostic module regimens, separately having different electro-diagnostic noninvasive units' for analysis with the same BMT.

Results: Bio-impedance curves estimated for schizophrenia with positive and negative signs differ in their amplitude. An interesting point was observed; patients due to protocol had sessions and they asked for extra, because of feeling better. It pinpointed on a favorable therapeutic effect. Epilepsy amplitude pattern was below amplitude of healthy control subjects. In relation to migraine, all patient receiving electrical bio-impedance units in the standardized treatment design, displaying a gradual fall in the impedance level as contrasted to bio-impedance SHAM control group display non-changing pattern of bioimpedance along the course of the trial.

Conclusion: This feasibility study indicates on high potentials of the BMT method. Since the study requires replication on larger samples engaging a multicenter research, the device must be accommodated with standardize treatment and diagnostic modules enabling objectively receiving non-invasive electrical bio-impedance dosimeter units. Such standardize practice, should broadly engage practitioners in objective measurements with safe and reliable parameters, advancing their practice with research in promoting personalize efficacy.

\title{
Caregiving Immediately after Stroke: A Study of Depressive Symptoms in Caregivers of Older Adults
}

\author{
Eeeseung Byun ${ }^{1, \varpi, \varkappa}$, Barbara Riegel ${ }^{2}$, Marilyn Sommers $^{2}$, Nancy Tkacs ${ }^{2}$ and Lois Evans ${ }^{2}$ \\ ${ }^{1}$ University of California, USA \\ ${ }^{2}$ University of Pennsylvania, USA
}

\begin{abstract}
Depression in caregivers puts them at high risk for subsequent morbidity and mortality. Little has been reported, however, about factors affecting depressive symptoms in the early post-stroke period, a time when stress and uncertainty about recovery of stroke survivors and a new role as caregiver are high. We conducted a prospective, longitudinal study and recruited 63 caregivers $(67 \%$ female, mean age $57 \pm 14$ years, $60 \%$ children) and their stroke-survivor relatives $(37 \%$ male, mean age $76 \pm 8$ years) from acute care settings. Caregivers were enrolled by 2 weeks post-stroke and revisited 4 weeks later. We measured caregivers' depressive symptoms using the Patient Health Questionnaire-9 (range: 0-24, higher scores = more severe symptoms) and collected caregiver and stroke-survivor characteristics. We also measured stress with the Perceived Stress Scale and salivary cortisol and uncertainty with the Perception of Uncertainty in Illness Scale. Unadjusted linear mixed models with repeated measures of depressive symptoms were computed. Although caregivers had mild depressive symptoms ( \pm \pm 6 at both time points), about $30 \%$ had at least moderate depressive symptoms at both time points. Caregiver uncertainty $(p<0.001)$, perceived stress $(p<0.001)$ but not cortisol, race $(p=0.022)$, income $(p=0.001)$, time spent to care $(p=0.039)$, coping $(p<0.001)$, social support $(p=0.006)$, and stroke-survivor functional status $(p=0.003)$ were associated with depressive symptoms. Factors confirmed in our study may help with timely identification of a subset of caregivers who are at highest risk for depressive symptoms and suggest areas for potential intervention.
\end{abstract}




\title{
A New Assessment for Episodic Memory. Episodic Memory Test and Caregiver's Episodic Memory Test
}

\author{
T. Ojea Ortega ${ }^{\square, \varkappa}$, M.M. Gonzalez Alvarez de Sotomayor and J. Romero Imbroda \\ Servicio de Neurología, Hospital Regional Universitario Carlos Haya, Spain
}

\begin{abstract}
Introduction and Objectives: to present the episodic memory test and the caregiver's episodic memory test, both accepted in 2012, and to define their characteristics through three different studies.

Methods and Results: The test consists of 10 questions about everyday facts that occurred the same day, the day before or more than a day before. It is highly sensitive and specific when distinguishing AD from episodic aMCI and from normal ageing, and it has well-defined cut-off values. A caregiver can administer it yielding similar results. An evolutionary comparative study showed that the episodic memory test detects $\mathrm{AD}$ in patients earlier than the MIS and the MMSE.

Conclusions: The episodic memory test is a simple test non-influenced by the patient's cultural level. It can be administered by the caregiver and detects AD earlier than the MIS and the MMSE, which help us conclude that verbal and visual memory are not equivalent to episodic memory.
\end{abstract}

\section{Collision or Convergence? Beliefs and Politics in Neuroscience Discovery, Ethics and Intervention}

\author{
Ben Paylor ${ }^{1, \varpi, \Perp, ~ H o l l y ~ L o n g s t a f f ~}{ }^{2}$, Fabio Rossi ${ }^{1}$ and Judy Illes ${ }^{2}$ \\ ${ }^{1}$ Biomedical Research Centre, University of British Columbia, Canada \\ ${ }^{2}$ National Core for Neuroethics, University of British Columbia Hospital, Canada
}

\begin{abstract}
Discovery and interventions for neurological disorders have a unique capacity to galvanize public opinion over issues of access, human rights, decision-making, and the definition of disease. The neuroscience community has a duty to explicitly recognize and address the powerful emotive capacity of neurological disease to galvanize stakeholders. The community must take heed of the importance of collective understanding surrounding experimental therapies as they emerge and inspire action that promotes understanding. Targeted communication strategies using the tools of knowledge translation, social media, and deliberative decision making will lead to better framed messages that co-originate with patient groups affected by neurological disorders, advance accurate reporting on regulatory principles for clinical trials, and mitigate controversies that are both sensitive and fundamental at an individual and societal level. . This presentation will highlight five cases where beliefs and politics prevailed over evidence and ethics. In doing so, it will examine lessons about the communication of risk and the power of public influence on science, society, and policy.
\end{abstract}

\section{Impact of Dopamine on T Cell Behavior of MS Patient}

\author{
Cleonice Bento $\square$ \\ Federal University of the State of Rio de Janeiro, Brazil
}

\begin{abstract}
Multiple sclerosis (MS) is a severe autoimmune disease that attacks the central nervous system. Some studies have indicated the involvement of Th1 and Th17 cells in the immunopathogenesis of MS. When they reach to the central nervous system, these cells are exposed to new conditions, such as the presence of neurotransmitters, including dopamine (DA). In this context, our recent findings have demonstrated that DA significantly elevated the polyclonal expansion of MS-derived T cells. Regarding the type of cytokine produced, this catecholamine increased not only the IFN- $\gamma$ but also Th17-related cytokines (IL-6, TNF- $\alpha$, IL-21 and IL-17). In contrast, DA reduced the release of anti-inflammatory cytokines IL-10 and TGF- $\beta$. Additionally, DA diminished the ability of corticoid in reducing IL-17 production by T cells from MS patients. Finally, in our system, the ability of DA in amplifying the production of IL-6 and IL-17 and reducing the release of IL-10 in cell cultures from MS patients was particularly dependent on CD4+ T cells and signaling through IL-6 receptor. Taken together, our results indicate that DA may
\end{abstract}


play a deleterious impact on MS pathogenesis by increasing Th17 cytokines release and reducing effectiveness of glucocorticoids actions.

\title{
Poster Abstracts:
}

\section{MTRNR2L12: A Candidate Blood Marker of Early Alzheimer's Disease-like Dementia}

\author{
Miroslaw Bik-Multanowski ${ }^{1, £, \AA}, J^{2}$ acek J. Pietrzyk ${ }^{1}$, Alina Midro ${ }^{2}$, Anna Madetko-Talowska ${ }^{1}$ and Agnieszka Grabowska ${ }^{1}$ \\ ${ }^{1}$ Department of Medical Genetics, Jagiellonian University Medical College, Poland \\ ${ }^{2}$ Department of Medical Genetics, Medical University, Poland
}

\begin{abstract}
Introduction: Histological changes constantly reported in autopsied brain tissue of adult patients with Down syndrome are identical with those observed in Alzheimer's disease. Interestingly, many adults with Down syndrome never present with cognitive deterioration whereas in others, Alzheimer's disease-like dementia develops already in the fifth decade of life.

The aim of the study was to identify variations of genome expression in blood that could serve as markers of early dementia in adults with Down syndrome.
\end{abstract}

Methods: Cognitive assessment was performed in a cohort of 48 adults with Down syndrome with subsequent microarraybased analysis of whole genome expression in leukocytes. The participants of the study were stratified with regard to their age and cognitive status to allow for comparison of group-specific expression profiles.

Results: Analysis of microarray data revealed highly significant differences between groups of younger patients with severe cognitive disability and of older patients without dementia with regard to expression of MTRNR2L12 gene, which is known to be associated with Alzheimer's disease. Subsequent comparison of another subgroup of younger patients with documented cognitive deterioration and the above group of older patients without dementia gave very similar results.

Conclusion: The findings of the study suggest a protective role of MTRNR2L12 in development of early Alzheimer's disease-like dementia in adults with Down syndrome. Further studies should be performed to evaluate potential usefulness of this marker in patients with Alzheimer's disease.

The study was sponsored by the Polish National Science Centre (DEC-2011/03/B/NZ5/01328).

\section{The Contributions of Game Therapy in Motor Performance of Individual with Cerebral Palsy}

\author{
Thiago Da Silva Dias ${ }^{1,2,3, \square, \varkappa, ~ K a r o l i n e ~ F a r o ~ D a ~ C o n c e i c ̧ a ̃ o ~}{ }^{2,3}$ and Rafael Luiz Morais Da Silva ${ }^{1,2,3}$ \\ ${ }^{1}$ MCPHS University, USA \\ ${ }^{2}$ Pará State University, Brazil \\ ${ }^{3}$ Nucleus of Assistive Technology and Accessibility Development, Brazil
}

\begin{abstract}
The videogame Nintendo $\mathrm{Wii}^{\circledR}$ has shown to have many contributions as a rehabilitation tool for individuals with neurological disorders. In this perspective, we hypothesized that the game therapy (GT), which involves using video games as a rehabilitation tool, would greatly contribute in the treatment of an individual with Cerebral Palsy (CP). This is a case study based on a broader experimental study, in which we used a pretest-posttest design to measure the participant's motor skills. The standardized protocol GMFM-88 (Gross Motor Function Measure) was applied before and after 10 GT sessions using the game Aladdin Magic Racer. The results supported our hypothesis, since the participant showed an improvement of $10.06 \%$ in the total score of the GMFM-88 after the GT sessions. Therefore, using the Nintendo Wii ${ }^{\circledR}$ contributed in the treatment by improving the participant's performance and, consequently, promoting a motor improvement. In addition, the motor tasks performed during the GT sessions enabled the emergence of motor patterns underlying activities involving the coordination of muscle groups (mainly wrist and elbow). In sum, the Nintendo Wii showed satisfying results, which were supported by a standardized assessment protocol. This suggests that the game therapy might be effective as a program of motor skills development for individuals with Cerebral Palsy and can be part of a rehabilitation program focused on these subjects regarding motor development.
\end{abstract}




\title{
Analysis of iPSC-derived Dopaminergic Neuron Susceptibility to Influenza and Excitotoxicity in Non-affective Psychosis
}

\author{
Jamileh Ahmed \\ University of South Florida, USA
}

\begin{abstract}
H1N1 virus susceptibility of iPSC-derived DA neurons from schizophrenia patients and controls will compared. C57/ BL-6 fibroblasts were reprogrammed into iPSCs using a lenti-viral vector containing SOKM genes. Pluripotency verification with the AP assay and immunocytochemistry ensured iPSC presence. The experimental outcome of ISPCs from DA neuron differentiation will be discussed in the Results section. Fibroblasts from patients and controls will be reprogrammed into iPSCs using a sendai virus vector containing SOKM. IPSCs will be characterized using the AP assay, immunocytochemistry, and RTPCR. IPSCs will then be differentiated into DA neurons. Gene methylation will be compared for both groups with customdesigned microarrays.
\end{abstract}

\section{The Effects of Mirror Therapy on the Gait of Subacute Stroke Patients: A Randomized Controlled Trial}

\author{
Myoung-kwon Kim ${ }^{1, \varpi, \mathfrak{i}}$ and Sang-gu Ji ${ }^{2}$ \\ ${ }^{1}$ Daegu Univeristy, Jilyang, Republic of Korea \\ ${ }^{2}$ University Hospital, Republic of Korea
}

\begin{abstract}
Objective: To investigate the effect of mirror therapy on the gait of patients with subacute stroke.

Subjects: Thirty-four patients with stroke were randomly assigned to two groups: a mirror therapy group (experimental) and a control group.

Interventions: The stroke patients in the experimental group underwent comprehensive rehabilitation therapy and mirror therapy for the lower limbs. The stroke patients in the control group underwent sham therapy and comprehensive rehabilitation therapy. Participants in both groups received therapy 5 days per week for 4 weeks.

Main Measures: Temporospatial gait characteristics, such as single stance, stance phase, step length, stride, swing phase, velocity, and cadence, were assessed before and after the 4 weeks therapy period.

Results: A significant difference was observed in post-training gains for the single stance (10.32 SD 4.14 vs. 6.54 SD 3.23), step length (8.47 SD 4.12 vs. 4.83 SD 2.14), and stride length (17.03 SD 6.57 vs 10.54 SD 4.34) between the experimental group and the control group $(p<0.05)$. However there were no significant differences between two groups on stance phase, swing phase, velocity, cadence and step width $(P>0.05)$.
\end{abstract}

Conclusion: We conclude that mirror therapy may be beneficial in improving the effects of stroke on gait ability.

\section{Which Type of Cane is the Most Efficient, Based on Oxygen Consumption and Balance Capacity, in Chronic Stroke Patients?}

\author{
Yeon- Gyu Jeong ${ }^{1}$ and Jung-Wan Koo ${ }^{2, \varpi, \AA}$ \\ ${ }^{1}$ Graduate School of the Catholic University of Korea, Republic of Korea \\ ${ }^{2}$ Department of Occupational and Environmental Medicine, Seoul St. Mary's Hospital, The Catholic University of Korea, Republic of \\ Korea
}

\begin{abstract}
Canes are widely prescribed as walking aids, but little is known about the effects of canes on the physiological cost of walking. The purpose of this study was to investigate the differences in oxygen consumption associated with the gaits of hemiplegic patients in terms of balance capacity according to the type of cane used. Twenty-nine patients with chronic stroke were divided into poor-balance $(n=15)$ and relatively-better-balance groups $(n=14)$ based on a cutoff score of 49 on the Berg Balance Scale
\end{abstract}


(BBS). Each patient completed three consecutive days of walking with a randomly assigned single-point cane, quad cane, or hemi-walker. We measured the oxygen expenditure and oxygen cost using a portable gas analyzer and heart rate during a 6-min walk test (6MWT) and a 10-m walk test (10MWT). The oxygen expenditure, gait endurance, and gait velocity were higher with the single-point cane $(p<0.01)$ than with any of the other cane types, and the oxygen costs were lower $(p<0.01)$ with the single-point cane among the patients with relatively better balance. The oxygen cost for the quad cane was lower $(p<0.01)$ than that found for any the other cane types among the patients with relatively poor balance. Our study revealed that single-point canes require less oxygen use at a given speed and permits greater speed the same oxygen consumption for hemiplegic patients with good balance. Walking aids with a greater base support may be more suitable than those with a smaller base support for patients with relatively poor balance.

\title{
The Effect of Smartphone-based Speed-interactive Treadmill Exercise Using Body Motion Tracking Technology on Gait in Stroke Patients
}

\author{
Changho Song ${ }^{1, \bullet, \dot{i}}$ and Kyoungjin Lee ${ }^{2}$ \\ ${ }^{1}$ Sahmyook University, South Korea \\ ${ }^{2}$ Texas Woman's University, USA
}

\begin{abstract}
The purpose of this study was to investigate the effects of smartphone-based speed-interactive treadmill exercise for improving of gait ability in chronic stroke patients. Thirty-four patients who experienced a stroke more than 6 months previously and who could walk independently participated in this study. Participants were randomly assigned to a speed-interactive treadmill exercise group (SITEG, $n=18$ ) or treadmill exercise group (TEG, $n=18$ ). SITEG received three 35 -min sessions per week of speed-interactive treadmill exercise for 6 weeks and TEG received three 35-min sessions per week of treadmill exercise without speed-interactive device for 6 weeks. Outcome measures were included the spatiotemporal gait parameter, gait stability and gait symmetry. SITEG and the TEG showed improvements in the spatiotemporal gait parameter, gait stability, but greater improvements were shown by the SITEG $(\mathrm{p}<.05)$. Smartphone-based speed-interactive device approach is feasible method to improve gait ability in chronic stroke patients.
\end{abstract}

\section{HIV-1 Vpr Disrupts Axonal Transport through Induction of $\alpha$-Synuclein}

\author{
Ying Wang ${ }^{\square, \imath}$ and Bassel E Sawaya \\ Temple University, USA
}

\begin{abstract}
Around 50\% of HIV-infected patients have neurological disorders with the mechanism unknown. Disruption of mitochondrial axonal transport, essential for the maintenance of synaptic and neuronal integrity and function, has long been known as a probable marker of neurodegenerative diseases. The effect of $\mathrm{HIV}-1$ proteins on mitochondrial disruptions remains unclear, therefore we sought to examine the impact of $\mathrm{HIV}-1$ viral protein $\mathrm{R}(\mathrm{Vpr})$ on mitochondrial functions. Using primary neuronal cultures, we demonstrated that $\mathrm{Vpr}$ reduced the motility of mitochondria, ATP production and mitochondrial biogenesis. Reduction in mitochondria motility was associated with alteration of microtubule stability, demonstrated by fragmented distribution of acetyl- $\Delta$-tubulin and increased tau aggregates, leading to impaired function of neurons. Loss of microtubule stability was accompanied with elevated expression of $\Delta$-synuclein protein. While overexpression of $\Delta$-synuclein mimicked the effect of $\mathrm{Vpr}$ in reducing acetyl- $\Delta$-tubulin, silence of it blocked the effect of $\mathrm{Vpr}$. Addition of recombinant $\mathrm{Vpr}$ also led to deregulation of Miro-1 and Parkin, proteins involved in mitochondrial trafficking. Using immunohistochemistry and western blot, similar results were obtained in hippocampus isolated from 18-month-old (aging) mice but not with 5-month-old (young) mice. This latest in particular and our data in general led to the conclusion that HIV-1 Vpr protein altered neuronal functions through inhibition of mitochondria transport and may accelerate neuronal ageing.
\end{abstract}

\section{Vitamin D Immunomodulatory Potential in Multiple Sclerosis}

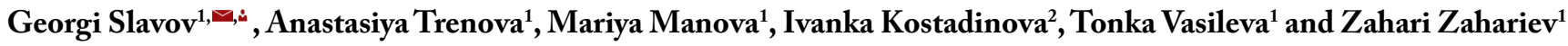 \\ ${ }^{1}$ Department of Neurology, Medical University, Bulgaria \\ ${ }^{2}$ Department of Pharmacology, Medical University, Bulgaria
}

Journal of Neurology \& Experimental Neuroscience | Volume 1 Supplement 1, 2015 


\begin{abstract}
Multiple sclerosis (MS) is an autoimmune disease of unknown etiology whose treatment is of limited efficiency. As it has been suggested that myelin destruction model, the clinical manifestation and the potential of therapeutic response in MS are correlated, it is quite justifiable that we study various factors (genetic, hormonal, and environmental) that take part in the autoimmune process in order to improve the control over the disrupted immune regulation. Results from epidemiological and clinical studies suggest that changes in vitamin D serum concentrations are correlated with the magnitude of the risk of developing MS, the phases of relapse and remittance and with gender differences in vitamin D metabolism. Experimental and clinical studies also have established that 25-hydroxy vitamin $\mathrm{D}(25(\mathrm{OH}) \mathrm{D})$ and 1,25 -dihydroxy vitamin $\mathrm{D}(1,25(\mathrm{OH}) 2 \mathrm{D})$ exert an immunomodulatory effect in the central nervous system and peripheral organs of the immune system. The standard reference range of vitamin $\mathrm{D}$ concentration in serum is $50-80 \mathrm{nmol} / 1$ it provides normal calcium metabolism. Issues that are discussed include the vitamin $\mathrm{D}$ serum concentration needed to suppress the aberrant immune response in MS patients; a subgroup of MS patients suitable for vitamin D treatment, the vitamin D being applied in optimally effective and safe dosage. MS prevalence rate in Bulgaria has increased two-fold in 17 years but this is a rather short interval to be able to assume that the gene pool of the population changes. Thus further studies on possible interactions between different environmental factors and their role in the disease pathogenesis are necessary.
\end{abstract}

\title{
Memory-enhancing and Neuro-protective Actions of N-Palmitoyl Serotonin in Scopolamine-Induced Amnesia Mice
}

\author{
Mee Ree Kim, ${ }^{\varpi}$, A Young Min and Choon Nan Doo \\ Department of Food and Nutrition, Chungnam National University, Korea
}

\begin{abstract}
$N$-Palmitoyl-5-hydroxytryptamines (Pal-5HT), an endocannabinoid, has recently been reported to express anti-allergic and anti-inflammatory actions, and ameliorate glutamate-induced cytotoxicity in HT-22 cells, independent of cannabinoid receptors. In this study, we examined the effect of Pal-5HT on deficits of learning and memory induced by scopolamine in mice. Memory performance was evaluated using the Morris water maze test and passive avoidance test. Additionally, acetylcholinesterase (AChE) and choline acetyltransferase (ChAT), oxidative stress biomarkers, and histological examination of neuronal cells in hippocampus were evaluated. The expression of brain derived neurotrophic factor (BDNF), phosphorylation of cAMP response element-binding protein ( $\mathrm{p}-\mathrm{CREB}), \mathrm{NAD}(\mathrm{P}) \mathrm{H}$ dehydrogenase [quinone] 1 (NQO-1), GCLC, iNOS and COX-2 were determined by western blotting. Immunoreactivity of BDNF was evaluated by double-labeling immunofluorescence.Pal5HT significantly improved amnesia, based on the Morris water maze test and the passive avoidance test. Pal-5HT effectively regulated cholinergic function. Pal-5HT suppressed oxidative stress, by elevating activities of antioxidant enzymes such as SOD, GPx, GR or NQO-1 as well as by lowering MDA level. Pal-5HT significantly prevented increased expression of iNOS and COX-2. Besides, neuronal cell death in hippocampal CA1 and CA3 regions were suppressed by Pal-5HT. Pal-5HT recovered expression of $\mathrm{p}-\mathrm{CREB}$ and BDNF, a neurotrophin in hippocampus. These results showed that Pal-5HT ameliorated memory and learning deficits through regulation of cholinergic function, activation of antioxidative defense systems, anti-inflammatory action as well as restoration of BDNF and p-CREB expression. It is suggested that Pal-5HT may be a potential candidate to treat some neurodegenerative diseases related to the deficit of memory such as AD.
\end{abstract}

\section{Transcranial Direct Current Stimulation (tDCS) Therapy for Major Depressive Disorder in HIV Population: A Safe, Effective Non-invasive Promising Treatment as Compared to Conventional Antidepressant Therapy}

\author{
Saeed Ahmed ${ }^{1}$, Hooria Manzoor ${ }^{2}$, Mariyah Z. Hussain ${ }^{3, \varpi, ょ, ~ S a t n e e t ~ S i n g h ~}{ }^{4}$, Sharmeen Amin ${ }^{5}$, Rizwan Ahmed ${ }^{6}$, Mustafa \\ Qureshi ${ }^{7}$, Swati Sood ${ }^{8}$ and Tazeen Azfar ${ }^{9}$ \\ ${ }^{1}$ Nassau University Medical Center, USA \\ ${ }^{2}$ Saint Vincent's Medical Center, Transplant Research Institute, USA \\ ${ }^{3}$ Foundation University Medical College, Pakistan \\ ${ }^{4}$ Cooper University Hospital, USA \\ 5 Jinnah Sindh Medical University Karachi, Pakistan \\ ${ }^{6}$ Liaquat Medical and Dentistry College, Pakistan \\ ${ }^{7}$ Brookdale University Hospital, USA \\ ${ }^{8}$ Dr. Rajendra Prasad Government Medical College, India
}


${ }^{9}$ Baqai Medical University, USA

\begin{abstract}
Major depressive disorder (MDD) is one of the most prevalent psychiatric disorders among the HIV-positive population. For long periods of time, MDD has been treated with conventional antidepressant therapy in these patients. Novel antidepressant therapy for Major depression in HIV infected adults is associated with various side effects such as sleep disturbance, nervousness, insomnia, weight loss, and sexual dysfunction that could lead to non-adherence to antiretroviral therapy. In order to uphold compliance and prevent patients from serious adverse effects of antidepressant therapy; emerging noninvasive innovative transcranial direct current stimulation (tDCS) could serve as a better alternative. tDCS has shown promising results; it is an effective, safe therapeutic strategy with an immediate onset of action as compared to conventional SSRI therapy. The application of tDCS is fairly an easy one and the device for conducting this treatment modality is rather highly portable. In addition to its convenient application, subjects who have been treated with (tDCS) in study settings, found it tolerable with minimum inconvenience and without developing chronic and profound adverse effects. Transcranial direct current stimulation does not have a limited role in addressing MDD alone in this population, however, it could be critical in helping these patients by decreasing their cravings for alcohol, smoking, and improve their cognition. This paper will further review this innovative treatment option and will also suggest tDCS to be studied in larger clinical trials as it could serve a huge role in developing better understanding of patient experiences regarding its tolerability, safety, and efficacy. Similarly, it will also provide important evidence to clinicians for development of better practices in this area.
\end{abstract}

\title{
Parkinson's Disease and Psychosis Treated with Clozapine
}

\author{
Raja Mahabaleshwar Hiremani ${ }^{\square, \varkappa}$, Arun Gupta and Christine Newland Smith \\ Flinders Medical Centre/Southern Adelaide Local Health Network, Australia
}

\begin{abstract}
Neuropsychiatric symptoms are a part of the non-motor symptoms related to Idiopathic Parkinson's disease (PD). The prevalence of psychosis in PD varies from 15.8 to $75 \%$ across various studies. Parkinson's disease associated psychosis (PDP) could manifest with visual hallucinatory experiences and delusional symptoms. The risk factors for psychosis in Parkinson's disease includes older age at disease onset, age greater than 65 years, longer duration of illness, more advanced disease, rapid eye movement sleep behavior disorder, higher doses of dopamine agonists, cognitive decline and reduced visual acuity in addition to other factors. Treatment of psychosis involves adjusting doses of Dopaminomimetic agents, improving sensory stimulation and antipsychotic medications. We present a patient with early onset (age of onset 54 years) Parkinson's disease who developed psychosis after 10 years of PD onset. He mainly had paranoid delusions as compared to delusions of jealousy, which is more common. His psychotic symptoms failed to respond to maximum recommended dose of Quetiapine $200 \mathrm{mg}$ BD but improved significantly with a trial of Clozapine. On clozapine $50 \mathrm{mg}$ his serum clozapine level was $50 \mathrm{ng} / \mathrm{mL}$.
\end{abstract}

\section{Increasing Self-awareness in Students with Autism}

\author{
Christine K. Duff \\ University of Central Florida, USA
}

\begin{abstract}
Neurobiological underpinnings of the limbic system have been linked with Autism, Joint Attention, concepts of 'self', and 'self-awareness'. Mizuno, Liu, Williams, Keller, Minshew and Just (2011), related the precuneous to 'self', and anterior insula to 'self-awareness'. Rosenberg and MacMaster (2009) reported Joint Attention was supported by the amygdala; and Mosconi, Cody-Hazlett, Poe, Gerig, Gimpel-Smith, and Piven (2009) found enlarged amygdalae in individuals with Autism.

Current descriptors of Autism include social difficulties that may be symptomatic of a debility in development of 'Self'(Duff and Flattery, 2014, published in the Journal of Autism and Developmental Disorders). A teaching methodology introduced Joint Attention strategies with reflected mirror images to help develop and increase awareness of 'self' in six students with Autism. These mirror based strategies measurably increased 'self-awareness' in students along four-step framework of Mirror Self Awareness. Concurrent associated third, second and first person pronoun acquisition appeared to indicate activation of limbic system function.
\end{abstract}




\title{
The Impact of Sleep Quality on Cognitive Functioning in Veterans "with Complex Medical Problems"
}

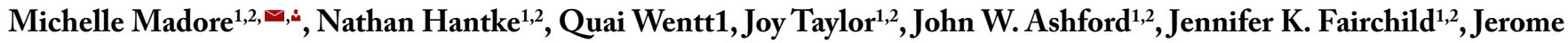 \\ Yesavage $^{1,2}$ and Maheen Adamson ${ }^{1,2}$ \\ ${ }^{1}$ War Related Illness Eऽ Injury Study Center (WRIISC) E VA Palo Alto Healthcare System, USA \\ ${ }^{2}$ Psychiatry E Behavioral Sciences, Stanford University School of Medicine, USA
}

\begin{abstract}
Poor sleep quality and insomnia are frequently reported concerns with prevalence rates ranging from 12 to $40 \%$ in older adults. Studies have shown that poor sleep quality continues to significantly impact cognitive performance in healthy older adults even after controlling for potentially confounding co-morbidities. Nebes and colleagues (2009) found that older adults with poor sleep quality performed worse compared to older adults with good sleep quality on measures of working memory, abstract problem-solving, attentional set-shifting and general cognitive status. The goal of the current study was to delineate the impact of self-reported sleep quality on cognitive functioning. We hypothesized poor sleep quality would account for difficulties in learning and memory. Sixty-eight Veterans (age: mean $=47.97, \mathrm{SD}=10.59$, range $=29-67$ ) with a history of complex medical problems were administered a cognitive screening battery. Self-report measures of post-traumatic stress disorder (PTSD), pain and sleep quality were also collected. Average scores on these measures suggested that these Veterans were experiencing moderate levels of symptoms consistent with PTSD, significant pain complaints and overall poor sleep quality. Sequential multiple regression analyses revealed that while both PTSD symptoms and poor sleep quality predicted performance for story learning, poor sleep was a better predictor of performance on this measure. These findings support the idea that sleep quality negatively impacts cognitive functioning. Furthermore, the results suggest in the context of multiple medical conditions, it may be helpful to provide treatments targeting improved sleep quality early in treatment planning, particularly in the aging Veteran population.
\end{abstract}

\section{Genetics and Migraine: Study on the Relationship between COMT and Migraine}

\author{
Svetlana Kantorovich ${ }^{\varpi}$ ¿, Natasha Anand, Brian Meshkin, Daniel A Schwarz, Bilikis Akindele, John Hubbard and \\ Tobore Onojighofia
}

Proove Biosciences, USA

\begin{abstract}
Background: Recent studies have implicated genes of the dopaminergic system in migraine. Catechol-O-methyltransferase $(\mathrm{COMT})$ is an enzyme that plays an important role in the metabolism of dopamine, epinephrine, and norepinephrine, and its genetic polymorphism influences the levels of these neurotransmitters and their metabolites.
\end{abstract}

Objective: The objective of this study is to investigate the role of the COMT polymorphism in genetic susceptibility to migraine.

Subjects: 49 subjects across 8 clinical research sites in the US. 24 diagnosed with migraine [ICD9 codes 346 series), mean age (44), males (2), females (22)], and 25 controls matched for race, gender and age.

Methods: Subjects were genotyped using Taqman ${ }^{\circledR}$ SNP Genotyping Assays (Life Technologies, Carlsbad, CA) for panel of 12 single nucleotide polymorphisms (SNPs) in genes encoding for proteins expressed in the mesolimbic reward pathway. These genes include: 5HT2a, 5-HTTL, COMT, ANKK1/DRD2, DRD1, DRD4, DAT, DBH, MTHFR, OPRK1, GABA-A receptor gamma2, and OPRM1.

Results: A cross tab analysis using IBM SPSS v21 found significant association between only COMT Val158Met (rs4680) and subjects with migraine [COMT: Dominant Model (G/G vs. G/A-A/A), Pearson chi-square p=0.011, Two sided fisher's exact 0.024 ]. COMT wild type $(\mathrm{G} / \mathrm{G})$ showed higher prevalence in subjects with migraine compared to controls. Logistic regression found that COMT G/G is more associated with migraine compared to the controls $(\mathrm{p}=0.016 \mathrm{OR} 5.923)$.

Conclusion: This study suggests that COMT Val158Met (rs4680) may play a role in genetic predisposition to migraine. Findings in this study will hopefully help improve understanding on the role of genetics in migraine. 


\title{
Genetics and Fibromyalgia:Study on the Relationshipbetween 5HT2aand Fibromyalgia
}

\author{
Svetlana Kantorovich ${ }^{\varpi} \Perp$, Natasha Anand, Brian Meshkin, Daniel A. Schwarz, Bilikis Akindele, John Hubbard and Tobore \\ Onojighofia
}

Proove Biosciences, USA

\begin{abstract}
Background: Fibromyalgia (FM) is a disorder characterized by widespread musculoskeletal pain, fatigue, memory loss, and mood issues. Genetics may play a role in risk of FM given that the odds of having FM are significantly increased for people who have an immediate family member afflicted with FM.
\end{abstract}

Objective: The objective of this study is to determine associations between genetics and risk of FM.

Subjects: 185 subjects across 10 clinical research sites in the US. 94 diagnosed with Fibromyalgia [ICD9 codes 729.1, mean age (49), males (20), females (74)], and 91 controls matched for age, race and gender.

Methods: Subjects were genotyped using Taqman ${ }^{\circledR}$ SNP Genotyping Assays (Life Technologies, Carlsbad, CA) for a panel of 12 single nucleotide polymorphisms in genes encoding for proteins expressed in the mesolimbic reward pathway. These genes include: 5HT2a, 5-HTTL, COMT, ANKK1/DRD2, DRD1, DRD4, DAT, DBH, MTHFR, OPRK1, GABA-A receptor gamma2, and OPRM1.

Results: A cross tab analysis using IBM SPSS v21 found a significant association between only 5-HT2a (rs7997012) and FM (5HT2a: Dominant Model (G/G vs. G/A-A/A) Pearson Chi-Square p=0.042, Two sided Fisher's Exact 0.044). Variants of 5-HT2A (G/A-A/A) were significantly more prevalent in subjects with FM compared to controls. Logistic regression found that 5HT2a variants are more associated with FM compared to the controls ( $\mathrm{p}=0.043$, OR 1.899).

Conclusion: This study suggests that 5-HT2a (rs7997012) may play a role in genetic predisposition to FM. Findings in this study will hopefully help further understand the role of genetics in FM and guide more comprehensive pharmacological approaches.

\section{Genetics and Insomnia: Study on the Role of DAT1 in Risk of Insomnia}

\author{
Svetlana Kantorovich ${ }^{\varpi,\llcorner, ~ N a t a s h a ~ A n a n d, ~ B r i a n ~ M e s h k i n, ~ D a n i e l ~ A ~ S c h w a r z, ~ B i l i k i s ~ A k i n d e l e, J o h n ~ H u b b a r d ~ a n d ~ T o b o r e ~}$ \\ Onojighofia
}

Proove Biosciences, USA

\section{Abstract}

Background: Evidence from studies suggests a degree of heritability for insomnia and for a reactivity of sleep patterns to stressful events. However, little is known of the role of genetics on insomnia, one of the most common sleep disorders.

Objective: The objective of this study is to determine the influence of genetics in insomnia.

Subjects: 86 pain patients from 10 research sites in the U.S. 42 diagnosed with Insomnia (ICD 9 Code 780.52) and 44 controls matched for age, gender and race.

Methods: Subjects were genotyped using Taqman ${ }^{\circledR}$ SNP Genotyping Assays (Life Technologies, Carlsbad, CA) for a panel of 12 single nucleotide polymorphisms in genes encoding for proteins expressed in the mesolimbic reward pathway. These genes include: 5HT2a, 5-HTTL, COMT, ANKK1/DRD2, DRD1, DRD4, DAT, DBH, MTHFR, OPRK1, GABA-A receptor gamma2, and OPRM1.

Results: A chi square test using SPSS V21 found only DAT1 (rs27072) to have a significant association with insomnia [DAT1: Dominant model (C/C vs. C/T-T/T), $\mathrm{p}=0.023$, Two sided Fisher's exact 0.030]. Further analysis using a binomial logistic regression $(\mathrm{p}=0.021)$ found that DAT1 $\mathrm{C} / \mathrm{C}$ is more associated with subjects with insomnia compared to a combination of the $\mathrm{C} / \mathrm{T}-\mathrm{T} / \mathrm{T}$ variations $(\mathrm{p}=0.026 \mathrm{OR}=3.176)$.

Conclusion: This study suggests that DAT1 may play a role in insomnia. As such, in addition to changes in both sleep mechanisms and stress-response-related gene-environment interactions influencing insomnia, this study hopes to help improve the understanding of the role of genetics in insomnia. 


\title{
Bifunctional Modulators of A $\beta$ Aggregation as Potential Agents for Alzheimer's Disease Therapy
}

\author{
Ming-Kuan Huª, , Chin-Lan Fu and Yei-Yang Yeh \\ School of Pharmacy, National Defense Medical Center, Taiwan
}

\begin{abstract}
Amyloid- $\beta(A \beta)$ peptide and its metal-mediated oligomerized states have been proposed to play an important role of the pathogenesis of Alzheimer's disease. Interruption of these metal-mediated amyloid peptides using metal-chelating functionalities implied considerable promise of a strategy for the treatment of the disease. Presented here are stilbene analogs that contain amyloid-binding and metal-chelating molecular motifs, which exhibited effective modulation on $A \beta$ aggregation in this study.

Two metal-mediated $A \beta$ aggregation studies were carried out in a buffer solution at $\mathrm{pH}$ 7.4. We investigated their abilities for the inhibition of forming metal-induced amyloid aggregation and the effects for the disruption of metal-induced aggregated fibrils. The degree of A $\beta$ aggregation in the presence of these stilbene analogs was also investigated by transmission electron microscopy. The resulting metal-chelating abilities and the images are presented.
\end{abstract}

\section{Rhinocerebral Mucormycosis with Top of Basilar Artery Syndrome}

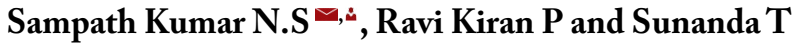 \\ Narayana Medical College and Hospital, India
}

\begin{abstract}
Mucormycosis is caused by fungi of the order mucorales and it is one of the fulminant and fatal mycotic infections which extends to the orbit and brain with high mortality rate. Mucormycosis is commonly reported in immunocompromised patients such as poorly controlled diabetes mellitus, blood dyscrasias, malnutrition, neutropenia, iron overload, organ transplant, and immunosuppressive therapy. Mucormycosis rises with an increase in incidence of diabetes mellitus and HIV infection leading to immunocompromised status of the patient. Mucormycosis has got rapid progression with high mortality, hence early recognition and aggressive treatment increase the survival rate. Here we report a case of rhinocerebral mucormycosis in an uncontrolled diabetic patient. Our aim is to highlight the varied forms of vascular dissemination of rhinocerebral mucormycosis resulting in ischemic arterial syndromes especially rare posterior ischemic circulatory strokes through dissemination of mucormycosis from sphenoid bone to clivus by bony erosion and to the basilar artery, thus causing the top of basilar artery syndrome.
\end{abstract}

\section{Eosinophilic Meningitis in a Child with Thalamic Infarct}

\author{
Suchetha Rao ${ }^{1, \varpi, \varkappa}$, Jayashree K ${ }^{1}$, Shivanada Pai $^{2}$ and Nutan Kamath ${ }^{1}$ \\ ${ }^{1}$ Department of Pediatrics, Kasturba Medical College, Manipal University, India \\ ${ }^{2}$ Department of Neurology, Kasturba Medical College, Manipal University, India
}

\begin{abstract}
Introduction: Eosinophilic meningitis (EM) is a rare clinical entity characterized by meningeal inflammation and eosinophilic pleocytosis in the cerebrospinal fluid (CSF). Invasion of the central nervous system by helminthic parasites, other infections as well as several non-infectious etiologies have also been associated. We report a child where EM is associated with thalamic infarct.

Case Report: A previously healthy 11 year old boy presented with fever, severe headache and vomiting of 10 days, difficulty in moving left upper and lower limb of 2 days. There was history of eating lizard. On admission child was febrile, his sensorium, cranial nerves were normal and there was no focal neurological deficit. His CSF analysis revealed normal protein and sugar, pleocytosis with $46 \%$ eosinophils. Magnetic resonance imaging of brain showed T2W/ FLAIR hyper intense area involving right posteromedial thalamus and patchy leptomeningeal enhancement on post contrast FLAIR images likely leptomeningtis. He was diagnosed to have eosinophilic meningitis with thalamic subacute infarct and treated with intravenous (IV) antibiotics, IV dexamethasone followed by oral prednisolone and albendazole $(15 \mathrm{mg} / \mathrm{kg} / \mathrm{day})$. Steroids and albendazole were given for a period of 21 days. Child gradually recovered.
\end{abstract}


Conclusion: Parasites are the most common causes of eosinophilic meningitis. History of exposure to the intermediate hosts should lead to a strong suspicion of these infestations. Early therapy with antihelminths and steroids is beneficial in such cases.

\title{
A Computational Model of Autism Disorder:Towards Developing Assistive'Technology to Improve Life's Quality
}

\author{
Reyes Juarez-Ramirez ${ }^{\varpi, \varkappa}$, Andres Mejia-Figueroa and Angeles Quezada \\ Universidad Autonoma de Baja California, Mexico
}

\begin{abstract}
Autism disorder has proliferated by this time in many countries. The main deficits presented in persons with this disorder are: language disability, social interaction and communication; repetitive behaviors. Many efforts have been made in order to assist autistic people; attention have been on behavioral and clinical aspects. Until today, there is a misconnection between clinical/medic and engineering efforts to have better solutions. Autistic people requires assistive technology to develop abilities and skills needed to deal with everyday activities. To develop assistive technology for these users, we propose a characterization of autism using a computational language such as the Unified Modeling Language (UML) in order to have a scheme to associate cognitive and physical capacities with human-computer interfaces. Our proposal includes a characterization modeling the kind of impairments at the level of the brain and the capabilities needed to execute everyday activities and to operate technological devices such as computers, mobile phones and tablets. Our contribution consists in a formal representation of autism details, which allows technology developers, especially software developers, to create adapted systems for autistic people. Our proposal is a result of working together with autism specialists and institutions which assist autistic people in the region on Baja California, Mexico. In this case, our work allows us gather different perspectives from psychologists, neurologists and computer scientists.
\end{abstract}

\section{Development of Novel Platelet Adhesion Inhibitor and Its Implication to Stroke}

\author{
Lin Zhang" \\ Department of Biochemical Engineering and Key Laboratory of Systems Bioengineering of the Ministry of Education, School of Chemical \\ Engineering and Technology, Tianjin University, China
}

\begin{abstract}
Arterial thrombus formation, initiated from the platelet adhesion on exposed collagen, is considered to cause stroke. In the present study, a biomimetic design strategy of platelet adhesion inhibitors was established, based on the exploration of molecular interactions between integrin $\alpha 2 \beta 1$ and collagen. First, a heptapeptide library containing 8000 candidates was designed to functionally mimic the binding motif of integrin $\alpha 2 \beta 1$. Then each heptapeptide in the library was docked onto collagen molecule for the assessment of its affinity, followed by a screening based on its structure similarity to the original structure in the affinity binding model. Eight candidates were then selected for further screening by molecular dynamics (MD) simulations. Thereafter, three candidates chosen from MD simulations were separately added into the physiological saline containing separated integrin and collagen, to check their abilities of blocking the integrin-collagen interaction using MD simulations. Of these three candidates, significant inhibition was observed in the presence of LWWNSYY. Finally, the binding affinity of LWWNSYY for collagen was demonstrated by isothermal titration calorimetry. Moreover, significant inhibition of platelet adhesion in the presence of LWWNSYY has been experimental validated. This work has thus developed an effective strategy for the biomimetic design of peptide-based platelet adhesion inhibitors, which would be helpful for the cure of stroke.
\end{abstract}




\section{Submit Your Next Manuscript}

We are currently accepting submissions from new authors. Read our inaugural issue articles and submit your next manuscript to Journal of Neurology and Experimental Neuroscience (JNEN).

Submit Online: http://www.editorialmanager.com/usg

Citation: Proceedings of the First Neurological Disorders Summit (NDS-2015). J Neurol Exp Neurosci 1(Suppl 1): S1-S51.

Copyright: This is an Open Access article distributed under the terms of the Creative Commons Attribution 4.0 International License (CC-BY) (http://creativecommons.org/licenses/by/4.0/) which permits commercial use, including reproduction, adaptation, and distribution of the article provided the original author and source are credited. Published by United Scientific Group.

Received: September 3, 2015 Accepted: September 21, 2015 Published: September 24, 2015 